\title{
Motion Detection in Dynamic Cardiac Positron Emission Tomography Images
}

by

Anbhu Sritharan, B.Eng.

A thesis submitted to the Faculty of Graduate and Postdoctoral Affairs in partial fulfillment of the requirements for the degree of

\section{Master of Applied Science}

in

\section{Biomedical Engineering}

Department of Systems and Computer Engineering

Carleton University

Ottawa, Ontario

(C) 2016, Anbhu Sritharan 


\begin{abstract}
Dynamic PET is being increasingly utilized to quantify myocardial blood flow, leading to improved diagnostic and prognostic value for the management of coronary artery disease. MBF quantification assumes constant positioning of anatomical structures throughout image acquisition. This assumption is violated in the presence of patient motion and heart motion. This thesis discusses methods for motion detection in dynamic cardiac ${ }^{82} \mathrm{Rb}$ PET images.
\end{abstract}

First, we determined if positron-emitting fiducial markers can be used as surrogate markers for heart motion in dynamic PET images. Second, algorithms were developed to track fiducial marker motion. Lastly, we tested the idea that principle component analysis may be able to detect heart motion in dynamic ${ }^{82} \mathrm{Rb}$ PET based on patterns in the residue image.

Overall, this thesis proposes low-cost, fast, and vendor independent post-reconstruction dynamic ${ }^{82} \mathrm{Rb}$ PET motion detection methods which can be used to detect motion in a clinical environment as a quality assurance tool. 


\section{Acknowledgements}

I would like to thank:

Dr. Ran Klein for giving me this amazing opportunity. This work would not be possible without your help. I express my appreciation for your support, guidance and encouragement over the past few years.

Dr. Andy Adler for his continued belief in our research and constant feedback.

Dr. Robert deKemp for the useful comments, remarks and engagement throughout the learning process of this thesis.

Chad Hunter for all the support he provided in generating simulation data for this research.

Hanif, Adrian, and all my colleagues at the University of Ottawa Heart Institute and the Ottawa Hospital who have played important roles in the completion of this work.

My lovely sisters, Jaline, Jeavana, Jeyasri, and Ashvinie for always believing in me throughout all my endeavours.

My friends, Atif, Baqar, Hassan, Kimo, LV, Mazen, Rohan, Saffatt, Sannan, Shady, Taz, and Zee, for your continued support and advice over the years

My parents for constantly pushing me to achieve bigger and better things and for supporting me throughout this entire process. I dedicate this thesis to the both of you. 


\section{Content}

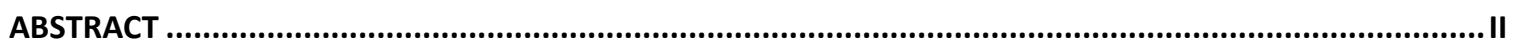

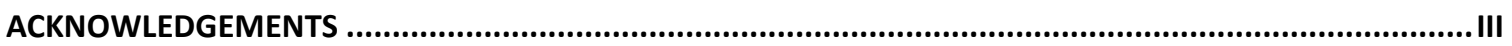

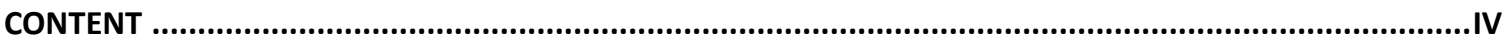

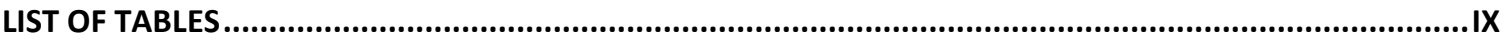

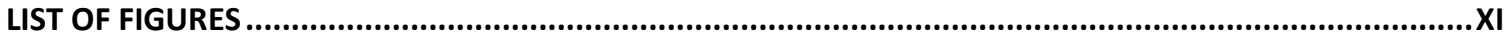

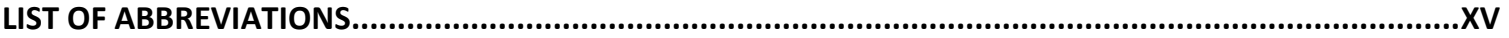

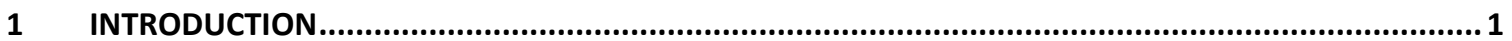

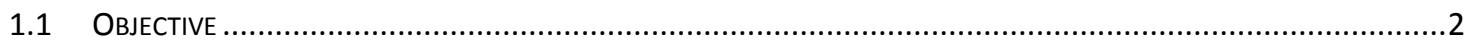

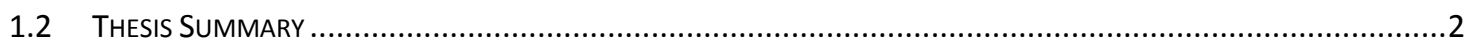

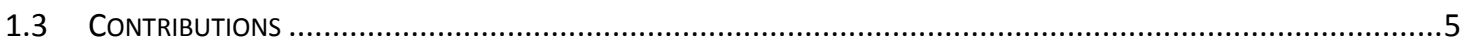

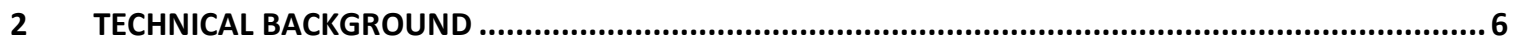

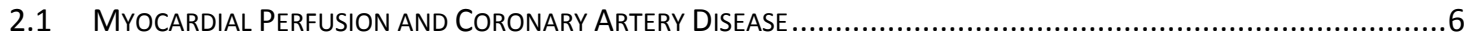

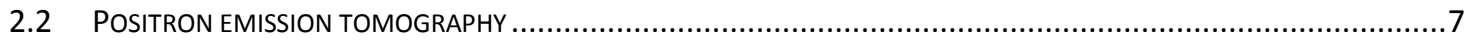

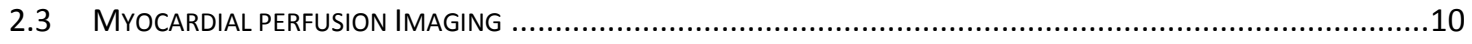

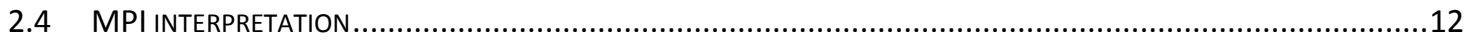

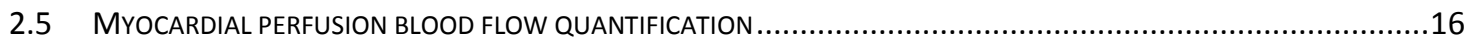

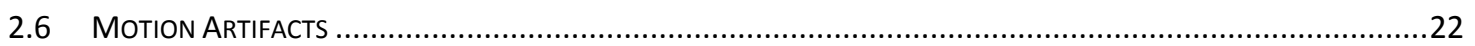

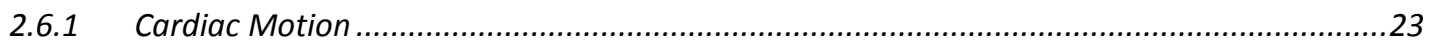

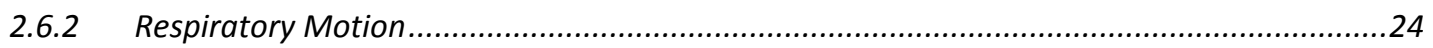

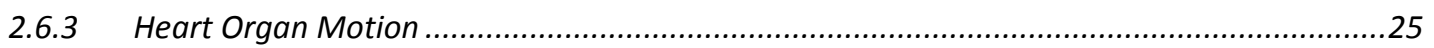

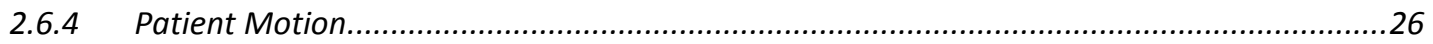


3 AGREEMENT BETWEEN CARDIAC AND FIDUCIAL MARKER MOTION DURING DYNAMIC CARDIAC

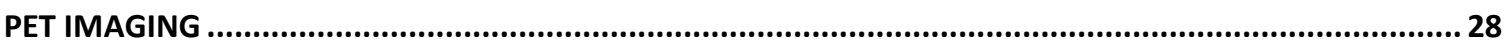

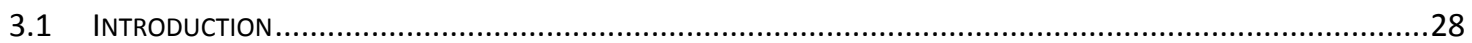

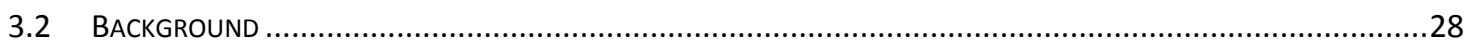

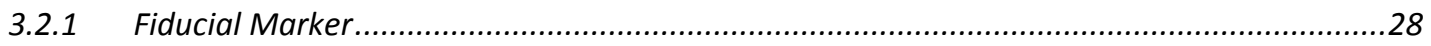

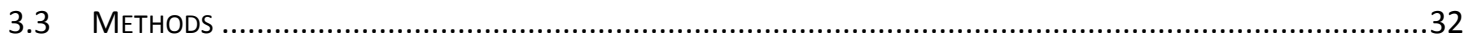

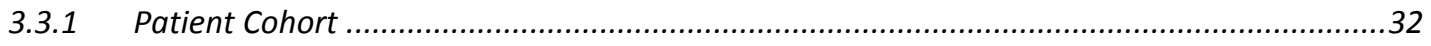

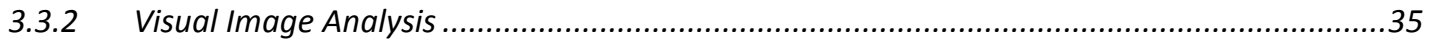

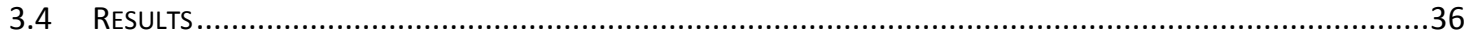

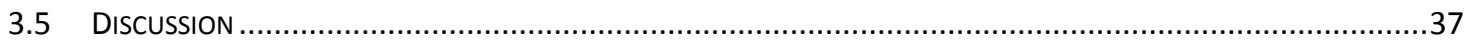

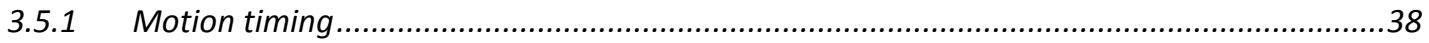

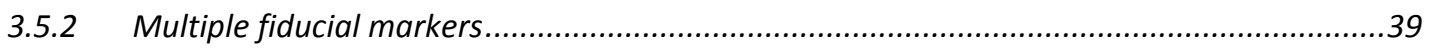

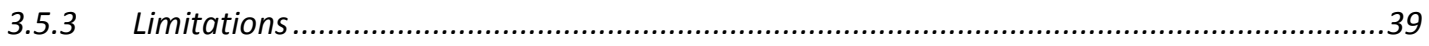

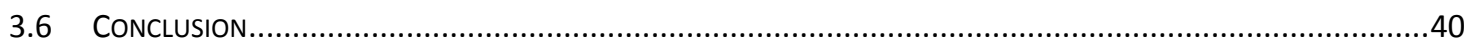

4 AUTOMATIC DETECTION OF PATIENT MOTION USING FIDUCIAL MARKERS IN DYNAMIC CARDIAC

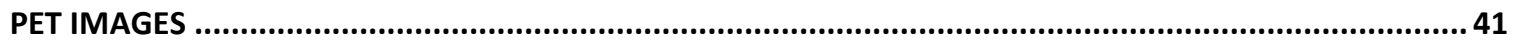

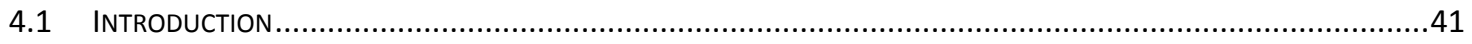

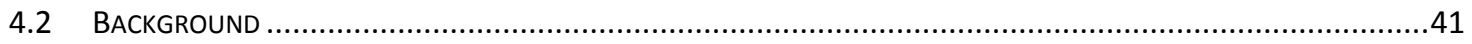

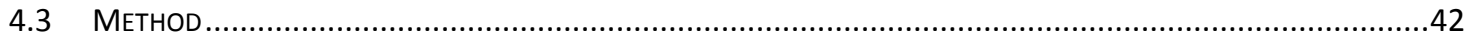

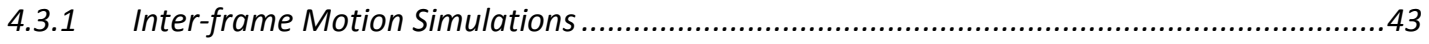

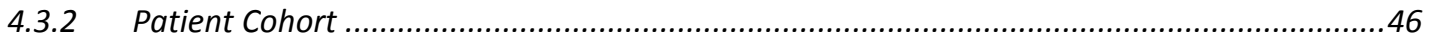

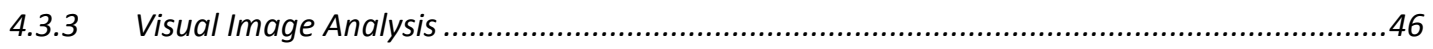

4.3.4 Automatic Fiducial Marker Motion Detection ..........................................................46

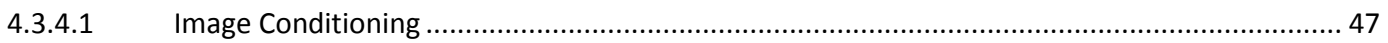




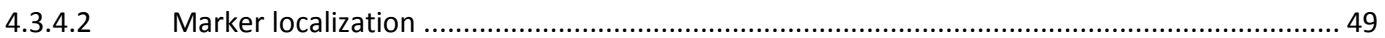

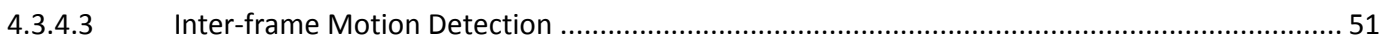

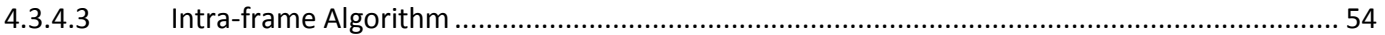

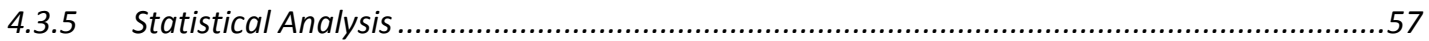

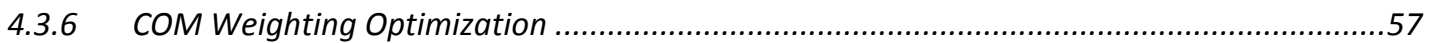

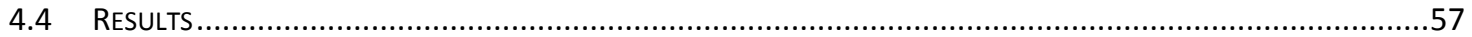

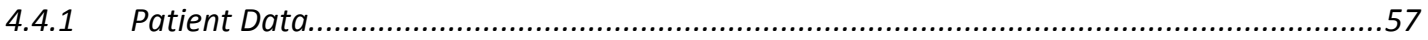

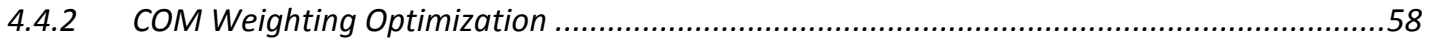

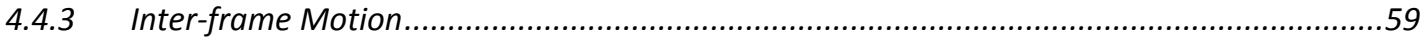

4.4.3.1 Simulation data - motion magnitude precision ............................................................... 59

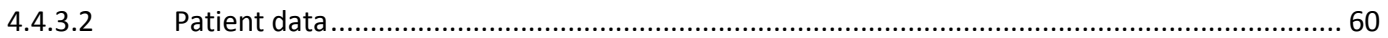

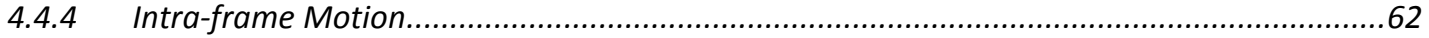

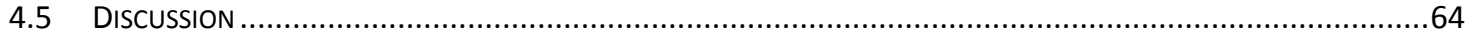

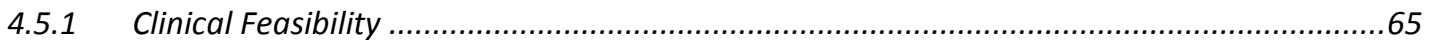

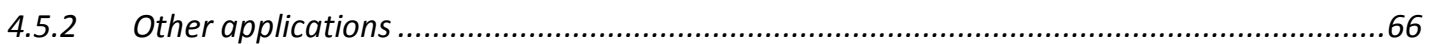

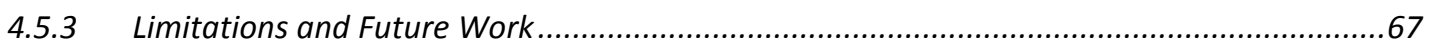

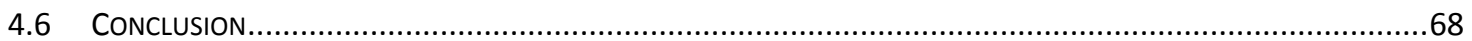

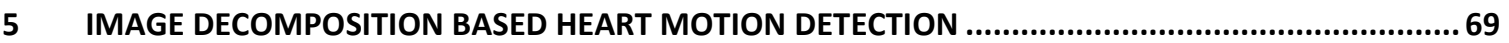

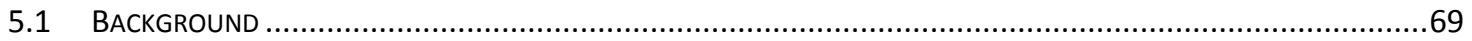

5.1.1 Matrix Decomposition - Dynamic Image Decomposition...........................................69

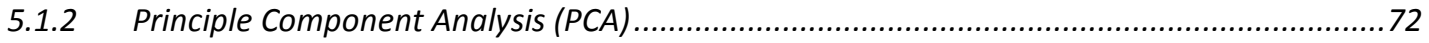

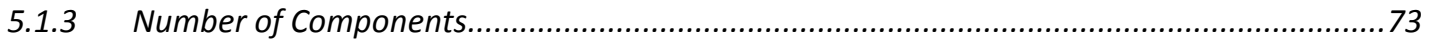

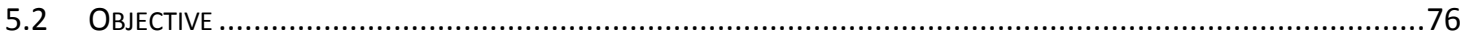

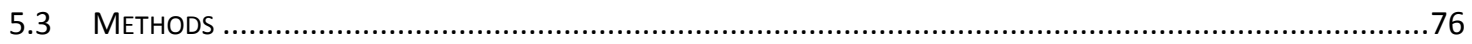

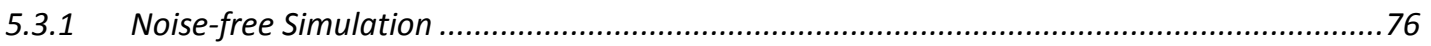

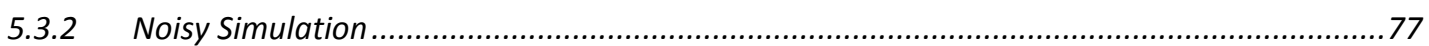




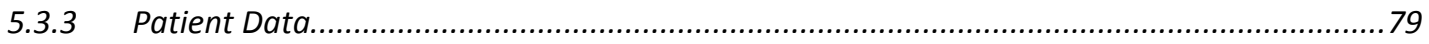

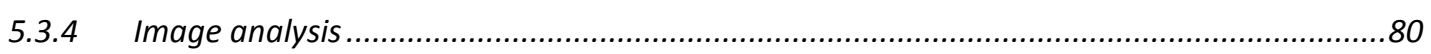

5.3.5 Automatic Motion Detection Using Quantification of Residue Signal.................................8 80

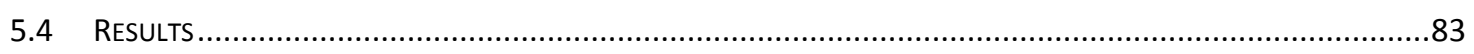

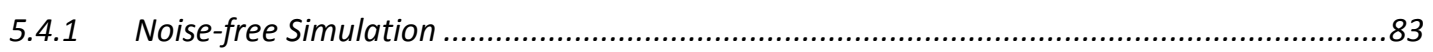

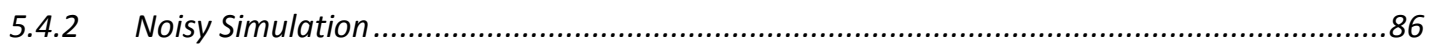

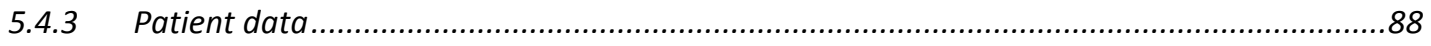

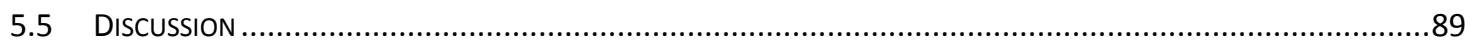

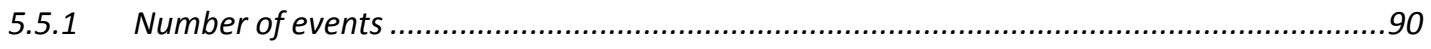

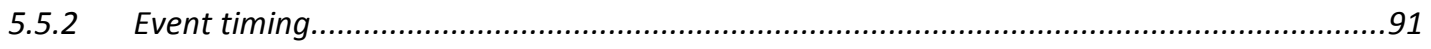

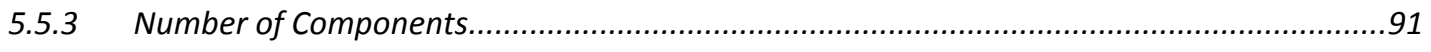

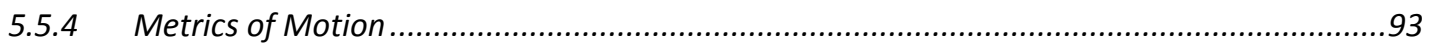

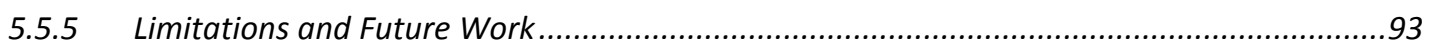

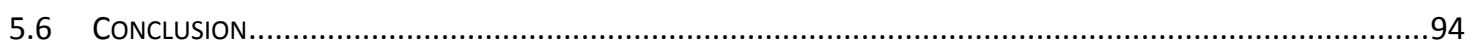

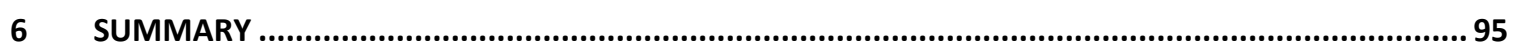

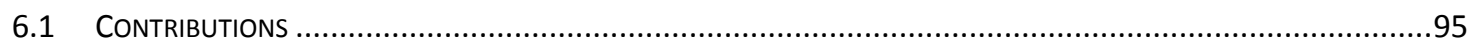

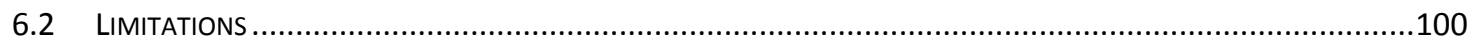

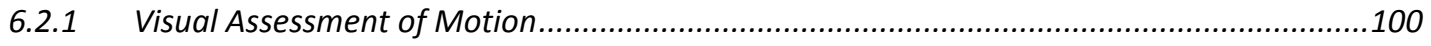

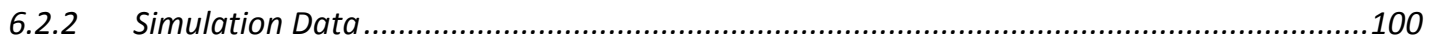

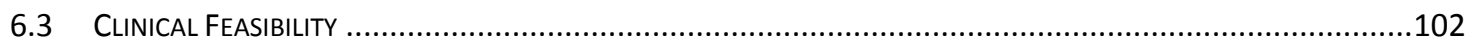

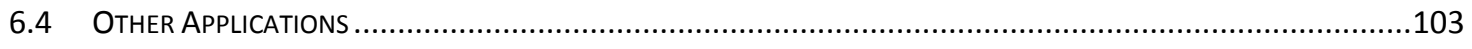

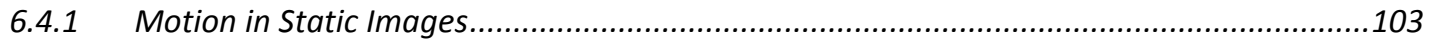

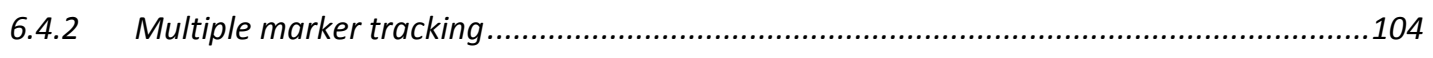

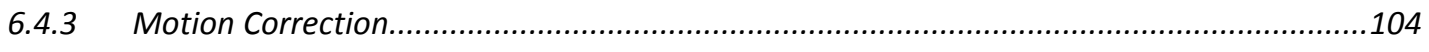

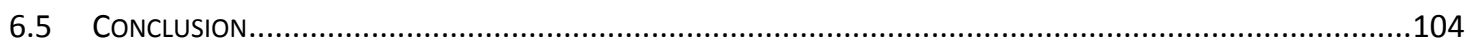




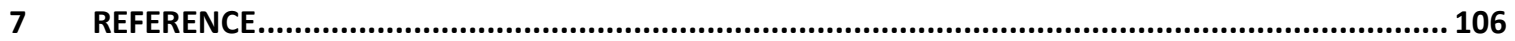

viii 


\section{List of Tables}

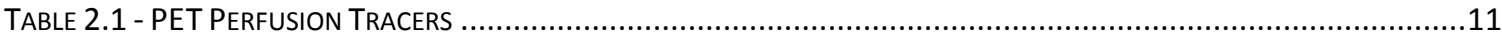

TABLE 3.1 - TIME FRAME NUMBERS AND THE CORRESPONDING TIME FRAME START TIME AND LENGTH TIME. .....................34

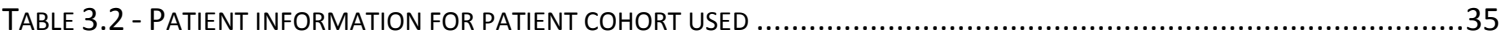

Table 3.3 - Presence of Patient and Heart motion in Patient Dynamic Pet images (n=130). Significant motion WAS DEFINED AS $\geq 2$ PIXELS

TABLE 3.4 - AGREEMENT BETWEEN MOTION TIME FRAME AND PRIMARY DIRECTION IN CASES WITH BOTH PATIENT AND HEART MOTION (N=24)

TABLE 4.1 - PERfoRMANCE OF INTER-FRAME MOtION DETECTION ALGORITHM (N=1820 FRAME PAIRS FROM 130 IMAGES)..61

TABLE 4.2 - Performance of INTRA-FRAME MOtION DETECTION ALGORITHM (N=1950 fRAMES IN 130 IMAGES) 63

TABLE 5.1 - DeteCted INTER-FRAME MOtION EVENTS IN 1 CM NOISE-FREE SIMULATED MOtION EVENTS. THE BOLDED VALUES ACCOUNT FOR PERFECT AGREEMENT BETWEEN RESIDUE ANALYSIS AND VISUAL ASSESSMENT. THE HIGHLIGHTED AREA ACCOUNTS FOR \pm 2 TIME fRAMES. $N=54$. NOte: Frame 2 AND 3 had NO MOtION SO ARE NOt SHOWN AND INTERFRAME MOTION CANNOT OCCUR IN TIME FRAME 1.

TABLE 5.2 - DeteCted INTER-FRAME MOtION EVENTS IN 2 CM NOISE-FREE SIMULATED MOTION EVENTS. THE BOLDED VALUES ACCOUNT FOR PERFECT AGREEMENT BETWEEN RESIDUE ANALYSIS AND VISUAL ASSESSMENT. THE HIGHLIGHTED AREA ACCOUNTS FOR \pm 2 TIME FRAMES. $\mathrm{N}=54$ 85

TABLE 5.3 - DeteCted INTER-FRAME MOtION EVENTS IN 1 CM NOISY SIMULATED MOTION EVENTS. The BOLDED VALUES ACCOUNT FOR PERFECT AGREEMENT BETWEEN RESIDUE ANALYSIS AND VISUAL ASSESSMENT. THE HIGHLIGHTED AREA ACCOUNTS FOR \pm 2 TIME FRAMES. $\mathrm{N}=54$ .88

TABle 5.4 - Detected INTER-FRAME MOtION EVENTS IN 2 CM NOISY SIMULATEd MOtiOn EVENTS. The BOLdED VALUES ACCOUNT FOR PERFECT AGREEMENT BETWEEN RESIDUE ANALYSIS AND VISUAL ASSESSMENT. THE HIGHLIGHTED AREA ACCOUNTS FOR \pm 2 TIME FRAMES. $\mathrm{N}=54$ .88 
TABle 5.5 - AutOMATIC teMPORAL localizATION OF INTER-FRAME MOtION EVENTS VS VISUAL ASSESSMENT. THE BOLDED VALUES ACCOUNT FOR PERFECT AGREEMENT BETWEEN RESIDUE ANALYSIS AND VISUAL ASSESSMENT. THE HIGHLIGHTED AREA ACCOUNTS FOR \pm 2 TIME FRAMES. $\quad \mathrm{N}=130$. NOTE: INTER-FRAME MOTION CANNOT OCCUR IN TIME FRAME $1 \ldots . .89$ 


\section{List of Figures}

FIGURE 2.1 - EXAMPLE DIAGRAM SHOWING A POLAR-MAP OF PERCENT UPTAKE FROM RCA, LCX, AND RCA WHICH PERFUSE TO THE LV MYOCARDIUM 7

FIGURE 2.2 - EXAMPLE REST AND STRESS CARDIAC PET UPTAKE IMAGES SHOWN IN SA, HLA, VLA PLANES. RED AREAS ARE HIGH IN ACTIVITY WHILE BLUE REGIONS HAVE LOWER ACTIVITY.

FIGURE 2.3 - EXAMPLE OF POLAR-MAP. THERE IS UPTAKE DEFICIENCY IN THE STRESS POLAR-MAP SHOWN BY THE BLUE REGION WHICH NORMALIZES AT REST. THE BOTTOM THREE POLAR-MAPS REPRESENT THE SUM-STRESS, SUM-REST, AND SUMDIFFERENCE SCORES WITH A LARGE REGION OF REVERSIBLE ISCHEMIA IN THE SEPTAL AND APICAL TERRITORIES OF THE LV. THIS PATTERN IS CONSISTENT WITH PARTIAL OBSTRUCTION OF THE LEFT-ANTERIOR-DESCENDING CORONARY ARTERY ....15

FIGURE 2.4 - TIME-ACTIVITY CONCENTRATION CURVES (TACS) (BOTTOM) SAMPLED FROM MYOCARDIUM AND BLOOD REGION OF INTEREST (TAN AND GREEN RESPECTIVELY) OVER A DYNAMIC CARDIAC ${ }^{82}$ RB PET IMAGE SEQUENCE. REPRESENTATIVE TIME FRAME IMAGES ARE SHOWN ON TOP WITH EACH TIME FRAME IMAGE NORMALIZED INDIVIDUALLY. 18

FIGURE $2.5-{ }^{82}$ RB PET ONE COMPARTMENTAL MODEL USED FOR MBF QUANTIFICATION. UPTAKE AND WASHOUT RATES OF TRACERS EXCHANGE BETWEEN ARTERIAL BLOOD AND MYOCARDIAL TISSUE ARE DEFINED BY CONSTANTS $\mathrm{K}_{1}$ [ML/MIN/G] AND K ${ }_{2}\left[\mathrm{MIN}^{-1}\right]$

Figure 3.1 - EXAMPLE of FiduCIAL MARKER IN A PET IMAge fRAME. THE INTENSITY OF THE PIXELS DECREASES MOVING RADIALLY OUTWARD FROM THE CENTER OF THE MARKER DUE TO LIMITED SPATIAL RESOLUTION OF PET IMAGES. .30

FIgURE 3.2 - TOP: FIDUCIAL MARKER MOTION BETWEEN TWO FRAMES AND RESULTING DISTORTION OF TIME-ACTIVITY CURVE BETWEen 5-6 Mins. BotTom: HeART ORGAN MOTION IN THE SAME DIRECTION AND TIME AS THE FIDUCIAL MARKER MOTION. THE CROSS-HAIR WAS NOT MOVED BETWEEN FRAMES IN BOTH IMAGES.

Figure 3.3 - COMPUted TOMOgRAPHY SLICE OF PATIENT IN THE TRANSVERSE PLANE. THE FIDUCIAL MARKER CAN BE SEEN ON THE OUTSIDE OF THE PATIENT'S CHEST. A SALINE BAG WAS PLACED OVER THE MARKER TO AVOID INTERFERENCE WITH THE SCATTER CORRECTION ALGORITHM. THE MARKER WAS PLACED AWAY FROM THE HEART TO AVOID INTERFERENCE WITH CLINICAL INTERPRETATION. 33 
FIGURE 3.4 - CLINICAL REST-STRESS ${ }^{82}$ RB PET/CT PROTOCOL USED FOR METHODS.

FIGURE 4.1 - WORKFLOW FOR DESIGN, IMPLEMENTATION, TESTING AND ANALYSIS OF THE INTER-FRAME MOTION DETECTION ALGORITHM .42

FIGURE 4.2 - WORKFLOW FOR DESIGN, IMPLEMENTATION, TESTING AND ANALYSIS OF THE INTRA-FRAME MOTION DETECTION ALGORITHM . .43

FIGURE 4.3 - NOISE-FREE SIMULATION MODEL. THE LEFT IMAGE SHOWS THE TRANSVERSE SLICE WHICH CONTAINS THE FIDUCIAL MARKER. THE RIGHT IMAGE SHOWS THE TRANSVERSE SLICE WHICH CONTAINS THE MYOCARDIUM IN THE SAME TIME FRAME. THE RED AREAS ARE HIGH ACTIVITY REGIONS AND THE BLUE AREAS ARE LOW-ACTIVITY REGIONS. .44

FIgURE 4.4 - FIDUCIAL MARKER CONTAINS MOST INTENSE PIXELS IN TIME FRAME 15. THEREFORE LOCALIZATION OF MARKER OCCURS IN THIS LAST TIME FRAME. .47

FIGURE 4.5 - GLOBAL (LEFT) AND LOCAL ROI ( $9 \times 9$ PIXELS) AROUND THE FIDUCIAL MARKER DEMONSTRATING BACKGROUND ACTIVITY SPILLING IN FROM THE PATIENT. THIS MARKER IS TAKEN FROM TIME FRAME 14 OF AN IMAGE WITH MOTION PRESENT. SPILL-IN ACTIVITY FROM THE PATIENT IS ALSO EVIDENT. .48

FIGURE 4.6 - 3D MESH-PLOT REPRESENTING THE PIXEL INTENSITIES OF A FIDUCIAL MARKER IN A 9X9 PIXEL VOLUME IN THE TRANSVERSE PLANE WITH SPILL-IN ACTIVITY FROM THE PATIENT (LEFT CORNER). .48

FIGURE 4.7 - 3D PLOT REPRESENTING THE PIXEL INTENSITIES OF A FIDUCIAL MARKER IN A 9X9X9 PIXEL VOLUME IN THE TRANSVERSE PLANE. FIDUCIAL MARKER PIXELS ARE MORE PROMINENT WITH BACKGROUND ACTIVITY SET TO 0. .49

FIGURE 4.8 - 3D PLOT REPRESENTING THE PIXEL INTENSITIES OF A FIDUCIAL MARKER IN A 9X9 PIXEL VOLUME IN THE TRANSVERSE PLANE. FIDUCIAL MARKER PIXELS HAVE LARGER PIXEL INTENSITIES AFTER BEING AMPLIFIED BY A POWER OF $N=9$. 51

FIGURE 4.9 - EXAMPLE OF INTER-FRAME ALGORITHM. THE TOP TWO IMAGES SHOW TWO SUCCESSIVE TIME FRAMES AND THEIR CORRESPONDING CENTER OF MASSES (COM). THE BOTTOM IMAGE IS OF THE TWO TOP IMAGES SUPERIMPOSED WITH THE DISTANCE (D) BETWEEN BOTH COM 52

FIGURE 4.10 - EXAMPLE OF INTER-FRAME MOTION REPORT. A 10 MM CHANGE IN COM IS INDICATED BETWEEN FRAME 10 AND FRAME 11. THE DISPLAY NOTATION IS SUCH THAT THE FIRST TIME FRAME IS THE REFERENCE (MOTION FREE)..............53

FIGURE 4.11 - A FUNCTION FITTED TO ACTIVITY FALL-OFF PROFILE TO ESTIMATE MARKER BLUR RESULTING FROM MOTION .....55 


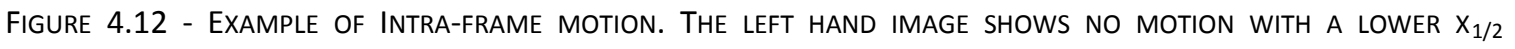
THRESHOLD AND PROFILE. THE RIGHT HAND IMAGE SHOWS AN EXAMPLE OF INTRA-FRAME MOTION WITH A HIGHER X $1 / 2$

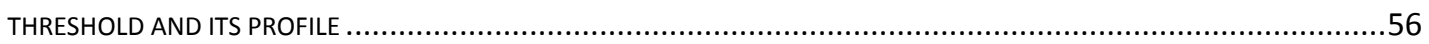

FIGURE 4.13 - INTER-FRAME MOTION DETECTION ACCURACY, SENSITIVITY AND SPECIFICITY FOR VARYING P POWER VALUES. ...58

FIGURE 4.14 - INTRA-FRAME MOTION DETECTION ACCURACY, SENSITIVITY AND SPECIFICITY FOR VARYING P POWER VALUES....59

FIGURE 4.15 - BOX PLOT SHOWING ERROR IN ALGORITHM REPORTED INTER-FRAME MOTION IN SIMULATED IMAGES WITH 0 AND 9.3750 MM SIMULATED MOTION (N=716 AND 54 RESPECTIVELY). THE MAXIMUM MOTION ERROR WAS LESS THAN 5\% PIXEL SIZE (3.125 MM). 60

FIGURE 4.16 - ROC ANALYSIS FOR INTER-FRAME ALGORITHM. THE BLUE CIRCLE REPRESENTS AN OPTIMAL THRESHOLD AT D=2.7 MM. 61

FIGURE 4.17 - ROC ANALYSIS FOR INTRA-FRAME ALGORITHM. THE BLUE CIRCLE REPRESENTS A THRESHOLD AT X $1 / 2=4.4$ MM. 62

FiguRE 4.18 - BOXPLOT DESCRIBING $X_{1 / 2}$ VALUES WHICH REPRESENT MOTION AND NO MOTION CASES. $\mathrm{X}_{1 / 2}=4.4 \mathrm{MM}$ THRESHOLD IS REPRESENTED BY THE BLACK LINE SEPARATING THE TWO MOTION CASES

FIGURE 5.1 - IMAGE DECOMPOSITION DIAGRAM: SUMMED PRODUCTS OF SPATIAL STRUCTURES AND TEMPORAL FACTORS (TOP LEFT) PROduCE AN ESTIMATED RECONSTRUCTED IMAGE SEQUENCE WHICH COMBINED WITH THE RESIDUE IMAGE SEQUENCE REPRESENTS THE ORIGINAL RAW DYNAMIC IMAGE SEQUENCE (BOTTOM). IMAGE DECOMPOSITION METHODS SIMULTANEOUSLY DETERMINE THE STRUCTURES AND FACTORS FROM THE RAW IMAGE SEQUENCE. THE RESIDUE WAS ANALYZED FOR PATTERNS INDICATING UNDESIRED SUBJECT MOTION DURING IMAGE ACQUISITION. 71

Figure 5.2: METHOD FOR SELECTING THE NUMBER OF COMPONENTS TO RESOLVE BASED ON TOTAL CUMULATIVE VARIANCE (Eigenvalues) AS Determined by PCA. The user Selects A Desired total VARIANCE (RANGe: 0-100\%) AND the CLOSEST NUMBER OF COMPONENTS TO ACHIEVE THIS THRESHOLD IS USED.

Figure 5.3 - AN EXAMPLE OF PCA IMAGE DECOMPOSITION USING 2 FACTORS IN A SIMULATED DYNAMIC IMAGE SEQUENCE WITH MOtion (LEFT). THE bLOOd AND MYOCARDIUM WALL PATTERNS CAN BE SEEN IN THE TWO COMPONENTS. A MYOCARDIUM STRUCTURE CAN ALSO BE SEEN AT IN THE LAST TWO TIME FRAMES OF THE RESIDUE IMAGE WHICH CORRESPONDS TO SIMULATED MOTION. NOTE THE SEPARATE COLOR SCALE ON THE RESIDUE IMAGE .75 


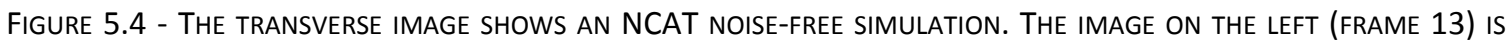
WITHOUT MOTION AND THE IMAGE ON THE RIGHT (FRAME 14) IS SHIFTED BY 2 CM IN THE +Y DIRECTION (AT 240 S). THE MYOCARDIUM CAN BE SEEN IN RED. THE CROSSHAIRS ARE FIXED BETWEEN TIME FRAMES. 77

Figure 5.5 - THE tRANSVERSE IMAGE SHOWS AN NCAT NOISY SIMULATION. THE IMAGE ON THE LEFT IS WITH NO MOTION AND THE IMAGE ON THE RIGHT IS SHIFTED BY 2 CM IN THE +Y DIRECTION (AT 240 S). THE MYOCARDIUM IS SHOWN IN RED. THE CROSSHAIRS WERE NOT SHIFTED BETWEEN FRAMES.

Figure 5.6 - RMS OF ACTIVITY IN EACH OF THE 15 TIME fRAMES OF NOISE-FREE SIMULATION IMAGES. TOP: MOTION WAS INTRODUCED BETWEEN TIME FRAME 3-4 IN THE +X DIRECTION WITH A MAGNITUdE OF 2 CM. MidDle: MotiON WAS INTRODUCED BETWEEN TIME FRAME 10-11 IN THE +X DIRECTION WITH A MAGNITUDE OF 2 CM. BOTTOM: MOTION WAS INTRODUCED BETWEEN TIME FRAME 14-15 IN THE +X DIRECTION WITH A MAGNITUDE OF 2 CM. THE RED CIRCLE REPRESENTS THE MAXIMUM RMS VALUE. 82

FIGURE 5.7 - RESIDUE OF NOISE-FREE NCAT PHANTOMS WITH SIMULATED MOTION (+2CM X DIRECTION) AT 30s, 60s, 80s, 90S, 120s, 150s, 180s, 240S AND 360S REPRESENTED BY THE DASHED LINE EACH IN RESIDUE. BLOOD POOL (EARLY TIME FRAMES) AND THE MYOCARDIUM (LATER TIME FRAMES) IS PRESENT BEFORE OR AFTER MOTION IS INTRODUCED. EACH IMAGE IS INDIVIDUALLY SCALED BETWEEN MINIMUM AND MAXIMUM INTENSITY PIXELS. . .84

FIGURE 5.8 - EXAMPLE RESIDUE OF NOISY NCAT PHANTOMS WITH SIMULATED MOTION AT 30, 60, 80, 90, 120, 150, 180, 240 AND 360 S REPRESENTED BY THE DASHED LINE IN EACH RESIDUE. BLOOD POOL (EARLY TIME FRAMES) AND THE MYOCARDIUM (LATER TIME FRAMES) LIKE PATTERNS ARE PRESENT BEFORE OR AFTER THE MOTION EVENT. 87

FiguRE 5.9 - RESIDUE OF PCA DECOMPOSITION USING 2, 3, 4 AND 5 FACTORS. THE NOISE-FREE SIMULATION CONSISTED OF 2 CM MOTION INTRODUCED AT 240 S (DASHED LINED)(BETWEEN TIME FRAME 13-14) IN THE +X DIRECTION 92 


\section{List of Abbreviations}

\begin{tabular}{|c|c|}
\hline${ }^{22} \mathrm{Na}$ & Sodium-22 \\
\hline $2 \mathrm{D}$ & Two dimensional \\
\hline $3 D$ & Three dimensional \\
\hline${ }^{82} \mathrm{Rb}$ & Rubidium-82 \\
\hline CAD & Coronary artery disease \\
\hline COM & Center of mass \\
\hline CT & Computed tomography \\
\hline FDG & ${ }^{18} \mathrm{~F}$-flurodeoxyglucose \\
\hline FOV & Field of view \\
\hline HLA & Horizontal long axis \\
\hline LAD & Left anterior descending artery \\
\hline LCx & Left circumflex artery \\
\hline LV & Left ventricle \\
\hline MBF & Myocardial blood flow \\
\hline MPI & Myocardial perfusion imaging \\
\hline
\end{tabular}




\begin{tabular}{|c|c|}
\hline MRI & Magnetic resonance imaging \\
\hline NCAT & Nurbs-based Cardiac-Torso \\
\hline NURBS & Noise-free non-uniform rational b-spline \\
\hline PCA & Principle component analysis \\
\hline PET & Positron emission tomography \\
\hline QC & Quality control \\
\hline $\mathrm{RC}$ & Tissue recovery coefficient \\
\hline RCA & Right coronary artery \\
\hline ROC & Receiver Operating Characteristic \\
\hline ROI & Region of interest \\
\hline RV & Right ventricle \\
\hline SA & Short axis \\
\hline SDS & Summed difference score \\
\hline SNR & Signal-to-noise ratio \\
\hline SPECT & Single photon emission computed tomography \\
\hline SRS & Summed rest score \\
\hline
\end{tabular}


TAC

TBF

VLA

VOI
Time-activity curve

Total blood fraction

Vertical long axis

Volume of interest 


\section{Introduction}

Coronary artery disease (CAD) is a leading cause of death in the western and developing world. This disease develops when the major arteries supplying blood to the heart muscle (myocardium) become damaged or diseased which can ultimately lead to myocardial infarction. Myocardial perfusion imaging (MPI) is used clinically to identify patients suffering of CAD and to guide therapy. However, MPI may miss uniform reduction in blood flow due to balanced disease in multiple coronary arteries and diffused disease of the micro vascular bed. Quantitative myocardial blood flow measurements (MBF) can overcome this limitation by improving the sensitivity of the test. The gold standard non-invasive modality for MBF quantification is positron emission tomography (PET). A PET camera is designed to detect photons associated with positron-emitting isotopes injected into the patient in trace molecular quantities (tracer). Thus, PET images correspond to the distribution of the tracer and therefore map the physiologic function of the body (i.e. MBF) with regard to the tracer molecule. MBF PET requires dynamic imaging as the tracer distributes through the body and is therefore susceptible to patient motion during image acquisition. Motion detection can be used to increase the confidence of MBF measurements in clinical use (quality control) and to flag cases where MBF may not be accurate due to the presence of motion.

One approach is to detect motion pre-reconstruction which requires the use of expensive external motion tracking equipment such as cameras and sensors to be 
incorporated in the clinical setting which can require changes to the imaging protocol and calibration $[1,2]$. While these systems can provide real-time measures, unique processing steps are required for integration with the PET data during image reconstruction which are specific to the camera vendor. A need remains for development of powerful post-reconstruction methods of motion detection which are less expensive, simple to integrate in clinical routine, and are vendor independent.

In this work, two post-reconstruction motion detection methods are proposed and their accuracy for flagging the presence of motion is evaluated. The first uses low-cost fiducial markers that is visible in the reconstructed image sequence and serves as a proxy for patient motion. The marker can be automatically tracked with high accuracy within the reconstructed dynamic image sequence. The second method uses principle component analysis to decompose the dynamic PET image sequence and the residue image can be visually or mathematically analyzed for evidence of heart motion.

\subsection{Objective}

The objective of this work is to develop a reliable post-reconstruction method for detecting the presence of undesired patient and heart motion in dynamic cardiac PET images for the purpose of quality control.

\subsection{Thesis Summary}

The background chapter gives an overview of the knowledge required to evaluate and meet the objective of this work. This chapter begins with an overview of myocardial 
perfusion and CAD and then describes how it can be visualized with PET. The chapter then discusses MPI and MBF quantification and how it is used in a clinical environment. Lastly, the background covers the various types of motion that could potentially be presented in PET images with an emphasis on heart organ and patient motions.

Chapter four evaluates whether there is a correlation between patient and heart organ motions during dynamic cardiac PET imaging. Patients undergoing dynamic cardiac Rubidium-82 PET imaging had a radioactive fiducial marker affixed to their chest during PET imaging. The dynamic image sequences were visually inspected, frame by frame, for both patient and heart motion using tailor made visualization software. Patient motion was deemed if the fiducial marker was observed to move in the dynamic image sequence. Time frame and motion-direction were recorded and analyzed for prevalence and concordance between patient and heart motion. While disagreement between the time frames and direction in which both fiducial marker and heart motion occurred was noted, it was concluded that fiducial markers have adequate sensitivity (89\%) and specificity (87\%) as predictors of presence of heart motion in dynamic image acquisition.

Chapter five describes automatic algorithms for detecting fiducial marker motion in dynamic cardiac PET images. Two post-reconstruction algorithms were developed to detect inter- and intra-frame fiducial marker motion respectively. Inter-frame motion was defined as motion between two successive frames and intra-frame motion as motion within a single frame, which is manifested as a directional blur of the marker. The fiducial marker was first detected within the image and then accurately located by 
using center of mass (COM) calculations. The inter-frame algorithm calculates the difference in COM between successive frames for each of the time frames, and compared against a pre-determined threshold to determine the presence of inter-frame motion. The intra-frame motion algorithm constructed profiles of activity fall-off with radial distance from the COM for each time frame in the dynamic image sequence. A modified-Gaussian curve was fit to each profile and a broadness parameter, B, was compared to a threshold value to determine presence of patient motion. The inter- and intra-frame algorithms using thresholds derived in this work had an accuracy of $91 \%$ and $87 \%$ respectively compared to visual assessment as a reference standard - adequate performance for automated motion detection for quality assurance.

In Chapter six we sought to evaluate an alternative and completely novel method to detect heart motion directly from the patient data, without the need for fiducial markers. Image decomposition was performed using principle component analysis (PCA) and the residue image (signal not described by the principle components) was visually and analytically interpreted for patterns indicating the presence of motion. Noise-free dynamic cardiac PET images were simulated with motion in various time frames and directions. The simulated images were decomposed and the residue images were visually evaluated for motion frames where potential motion was present.

The final chapter discusses the implications of this work to this and other related applications. It describes future direction including applications. We conclude that automated fiducial motion tracking is feasible for flagging the presence of motion. While 
a single fiducial marker is not suitable for characterizing motion and driving motion correction.

\subsection{Contributions}

Part of this work was presented at international meetings and published:

- A. Sritharan, R. deKemp, R. Beanlands, A. Adler, and R. Klein, "Automatic Detection of Patient Motion Using Fiducial Markers in Dynamic Cardiac PET Images," J Nucl Med, vol. 56, no. supplement 3, pp. 133-133, May 2015.

- A. Sritharan, A. Adler, R. deKemp, R. Beanlands, and R. Klein, "Agreement between patient and heart motion during dynamic cardiac PET imaging," $J$ Nucl Med, vol. 55, no. supplement 1, pp. 1785-1785, May 2014. 


\section{Technical Background}

\subsection{Myocardial Perfusion and Coronary Artery Disease}

The heart is a vital organ pumping blood to the lungs and body. This work requires continued supply of nutrients, energy, and oxygen via the blood through a network of arteries. The largest of these arteries are the coronary arteries which originate at the aortic root and bifurcate into smaller and smaller vessels, ending in the capillary bed. The myocardium (heart muscle) contains cardiomyocytes which contract the heart. The myocardium is thickest $(\sim 1 \mathrm{~cm})$ in the left ventricle (LV) due to the LV having to pump blood into the aorta and through the body requiring a large amount of pressure. Consequently even with limited image spatial resolutions, the LV is a thick enough target for non-invasive nuclear imaging modalities. The right ventricle $(\sim 5 \mathrm{~mm})$ and atrial walls

( 2 $\mathrm{mm}$ ) are more difficult to image, but are also being increasingly images as the spatial resolution of improves. Figure 2.1 illustrates the most common anatomy of the main coronary arteries (Left anterior descending artery [LAD], Left circumflex artery [LCX], Right coronary artery [RCA]) which perfuse the LV. The polar-map at the center of Figure 1 shows the percentage of tracer uptake in the LV. The blue region represents regions of low tracer uptake and therefore low blood uptake while the red regions represent regions of high tracer uptake associated with normal regional blood supply. 


\section{Anterior}
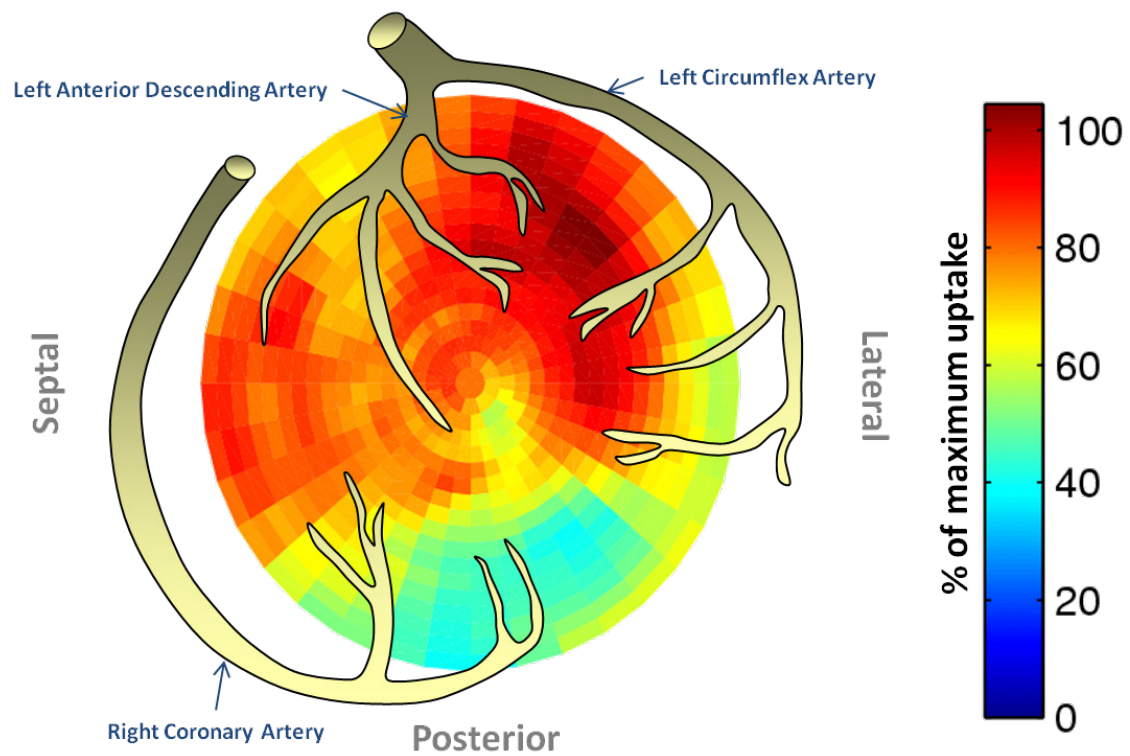

Figure 2.1 - Example diagram showing a polar-map of percent uptake from RCA, LCx, and RCA which perfuse to the LV myocardium

Coronary artery disease (CAD) develops gradually, leading to narrowing of the main coronary arteries which eventually limit the supply of blood to the myocardium and therefore limit tracer uptake by the myocardium. CAD is one of the leading causes of mortality and morbidity in western society and is increasing in prevalence in developing societies [3]. With proper diagnosis and intervention however, quality of life and survival can be increased significantly. Positron emission tomography (PET) is a sensitive and specific imaging modality for determining severity and extent of CAD in patient clinical setting [4].

\subsection{Positron emission tomography}

Positron emission tomography (PET) imaging has emerged as a superior alternative for myocardial perfusion imaging (MPI) compared to SPECT. It's superiority to SPECT was 
recognized due to technological advancements that result in more accurate molecular imaging and quantification [5]. A PET camera uses detectors all around the patient in a ring formation and does not require the use of physical collimators, as in SPECT, allowing for higher sensitivity of the camera to detected emitted radiation [5]. Therefore PET can detect a larger number of emitted photons which results in improved image quality including higher signal-to-noise ratio (SNR), superior spatial resolution and shorter scan times [5].

PET using ${ }^{18}$ F-flurodeoxyglucose (FDG) has been widely adopted as the gold standard for sensitive and specific detection and staging of cancer, and for assessing response to therapy [6] and has been the dominant clinical application of PET. Cardiac PET has been growing dramatically in recent years mostly using ${ }^{82} \mathrm{Rb}$, which has excellent imaging properties and can be cost effective in high patient volume clinics. ${ }^{82} \mathrm{Rb}$ PET has a higher sensitivity and specificity in the detection of obstructive CAD compared to SPECT [7]. A recent meta-analysis study showed that ${ }^{82} \mathrm{Rb}$ PET and SPECT had a sensitivities of $88 \%$ and $85 \%$ and specificities of $90 \%$ and $85 \%$ respectively [8]. This is due to PET images having a higher spatial resolution, reduced image noise, accurate attenuation correction, and higher tracer extraction by the myocardium [9].

A PET camera is designed to detect photons which are produced from positron-emitting isotopes injected into the patient. Thus PET images correspond to the distribution of the tracer and are associated with the physiologic function of the body with regard to the tracer molecule, as opposed to anatomy, as with x-ray, ultrasound and magnetic 
resonance imaging. Current industry limitations to widespread adoption of PET is the high cost of the camera compared to SPECT and the potential need for an on-site cyclotron depending on what isotope is required for imaging [10].

A positron-emitting radioactive tracer is administered into the body and distributes based on physiologic functions. As the radioisotope label decays a positron is emitted which slows and annihilates with a nearby electron, producing two coincident $511 \mathrm{keV}$ gamma rays (photons) travelling in opposite directions (collinear). The PET camera is composed of detectors designed to absorb and detect these photons. Each of the detectors is linked so when two coincident photons hit the detectors within a restricted time window it is registered as a coincident event. Since both coincident photons move in opposite directions the coincident event is assumed to have occurred along the line of response (LOR) between the detector pair. Using complex image reconstruction algorithms (e.g. filtered back projection or iterative optimization) PET can create tomographic images by determining where the coincident events were most likely to originate along the LORs. The final image is a volume consisting of many slices through of the field of view (FOV) of the camera. The amount of radiation emitted from a finite volume is proportionate to the tracer concentration.

An advantage of PET is its accurate attenuation correction [7] along with ability to correct for other physical phenomenons (e.g. random and scatter events) prior-to or as part of image reconstruction to achieve quantitatively accurate images of tracer distribution. Due to the uniquely high sensitivity of PET, a trace (on the scale of nano- to 
femto-moles) amount of this radioactive compound is required, which does not invoke a physiologic response - hence the name tracer.

\subsection{Myocardial perfusion Imaging}

Myocardial perfusion imaging (MPI) typically consists of imaging both at states of cardiac rest and stress in order to assess the ability to increase perfusion to meet increased oxygen demands with exercise. Cardiac stressing is typically performed either through physical exercise or through the use of pharmacological drugs such as adenosine, dipyridamole, and dobutamine $[9,11,12]$. The rest and stress images are interpreted together. In each image, the region of highest activity is assumed to be normally perfused and all other regions are compared to it. Regions with reduced tracer uptake (defects) are deemed to have reduced perfusion compared to the reference region. If regions at both stress and rest have reduced uptake then the region is considered to be scarred. A region with reduced uptake at stress but normal uptake at rest is considered to be ischemic [10].

There are a wide variety of tracers which can be used to probe different biochemical processes in the body ranging from myocardial perfusion, cell signaling, lesion tracking and metabolic processes making PET a valuable imaging modality in various fields. MPI tracers are designed to have good binding affinity to the myocardium where it is taken up by the tissue in relation to the rate of blood flow perfusing the tissue and is retained in the tissue, making it a good perfusion tracer [10]. 
An important property of any tracer is its rate of radioactive decay. The half-life of a radioactive compound is defined by the time it takes for half of the original activity to decay. Short lived tracers have the benefits of reducing potentially harmful radiation exposure to the patient and allows for quick successive testing such as for imaging at states of cardiac rest and stress. However, when using short lived tracers (e.g. ${ }^{82} \mathrm{Rb}$ ) patients may need to be stressed pharmacologically as opposed to physical exercise. Table 2.1 shows the production methods of commonly used PET MPI tracers and their half-life.

Table 2.1 - PET Perfusion Tracers

\begin{tabular}{|c|c|c|c|}
\hline Isotope & Half-Life $\left(\mathbf{T}_{\mathbf{1} / \mathbf{2}}\right)$ [min] & Production & MPI Tracer \\
\hline${ }^{82} \mathrm{Rb}$ & 1.3 & Generator & ${ }^{82} \mathrm{Rb}$-chloride \\
\hline${ }^{13} \mathrm{~N}$ & 10.0 & Cyclotron & ${ }^{13} \mathrm{~N}$-ammonia \\
\hline${ }^{18} \mathrm{~F}$ & 110 & Cyclotron & ${ }^{18} \mathrm{~F}$-Flurpiridaz \\
\hline${ }^{11} \mathrm{C}$ & 20.4 & Cyclotron & ${ }^{11} \mathrm{C}$-acetate \\
\hline${ }^{15} \mathrm{O}$ & 2.0 & Cyclotron & ${ }^{15} \mathrm{O}$-water \\
\hline
\end{tabular}

Most biological compound can be labelled with ${ }^{11} \mathrm{C}$ and ${ }^{15} \mathrm{O}$ for PET imaging and thus seem as attractive labelling isotopes. However, as with most tracers that are cyclotron produced they can be expensive to produce and require coordination between the imaging staff and the cyclotron lab due to their short radioactive half-lives. ${ }^{18} \mathrm{~F}$ has a half-life of 2 hours and is thus the exception to the rule. Currently there are no 
approved ${ }^{18} \mathrm{~F}$ based MPI tracers, but Flurpiridaz is in phase-3 of clinical trials $[13]{ }^{13} \mathrm{~N}$ ammonia is the most commonly used cyclotron produced MPI tracer and has excellent imaging properties, with the exception of high liver and lung uptake, which can interfere with interpretation of cardiac regions [14]. ${ }^{13} \mathrm{~N}$-ammonia has a 10 minute half-life and therefore also requires and on-site cyclotron.

${ }^{82} \mathrm{Rb}$ is a short lived (76s half-life) generator produced isotope that does not require an on-site cyclotron. $\mathrm{A}^{82} \mathrm{Sr} /{ }^{82} \mathrm{Rb}$ generator lasts $4-8$ weeks and can be used as an onsite ${ }^{82} \mathrm{Rb}$ source every 10 minutes allowing for a high patient throughput [15]. ${ }^{82} \mathrm{Rb}$ is an analog of potassium which is required for muscle contractions. Specifically the $\mathrm{Na}^{+} / \mathrm{K}^{+}$ ATPase actively pumps in ${ }^{82} \mathrm{Rb}$ into the myocardial tissue [10]. ${ }^{82} \mathrm{Rb}$ uptake is thus dependent on the rate of blood flow to the myocardium.

For MPI, image acquisition occurs $\sim 2$ minutes post-injection of the tracer allowing the tracer sufficient time for distribution, uptake and retention in the myocardium. Image acquisition lasts for several minutes in order to detect enough coincidence events to reconstruct a low-noise image.

\subsection{MPI interpretation}

Volume images of the myocardium, by convention, are reoriented to a fixed reference frame of the left ventricle myocardium. Series of slices in three orthogonal planes are displayed for interpretation. The short axis (SA), vertical long axis (VLA) and horizontal long axis (HLA) are demonstrated in Figure 2.2. MBF can be sampled using specialized 
software and displayed in a polar-map format as demonstrated in Figure 2.3 with the apex at the center and the base radially outward, with the septal wall to the left and the inferior wall at the bottom of the polar-map [16]. 


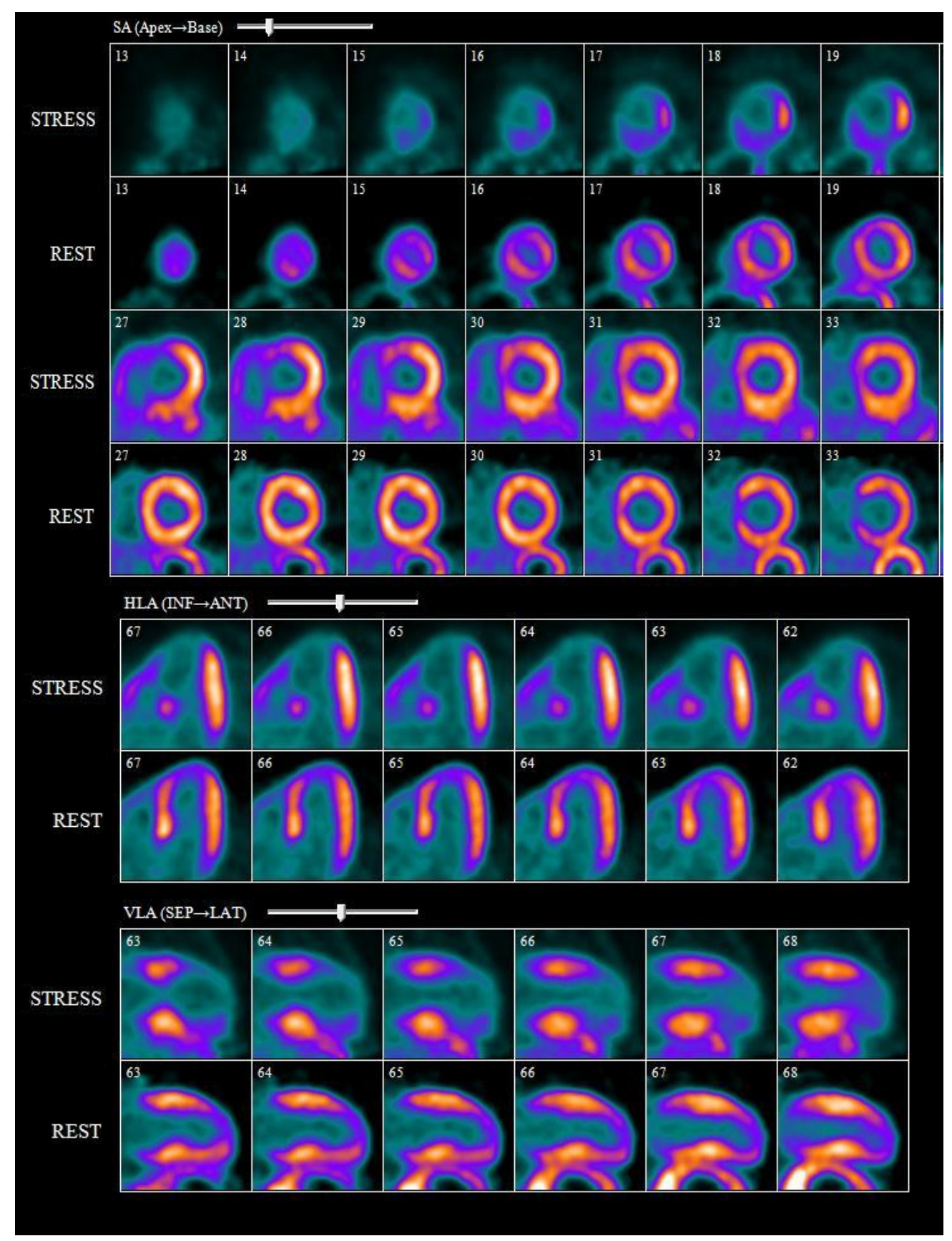

Figure 2.2 - Example rest and stress cardiac PET uptake images shown in SA, HLA, VLA planes. Red areas are high in activity while blue regions have lower activity. 


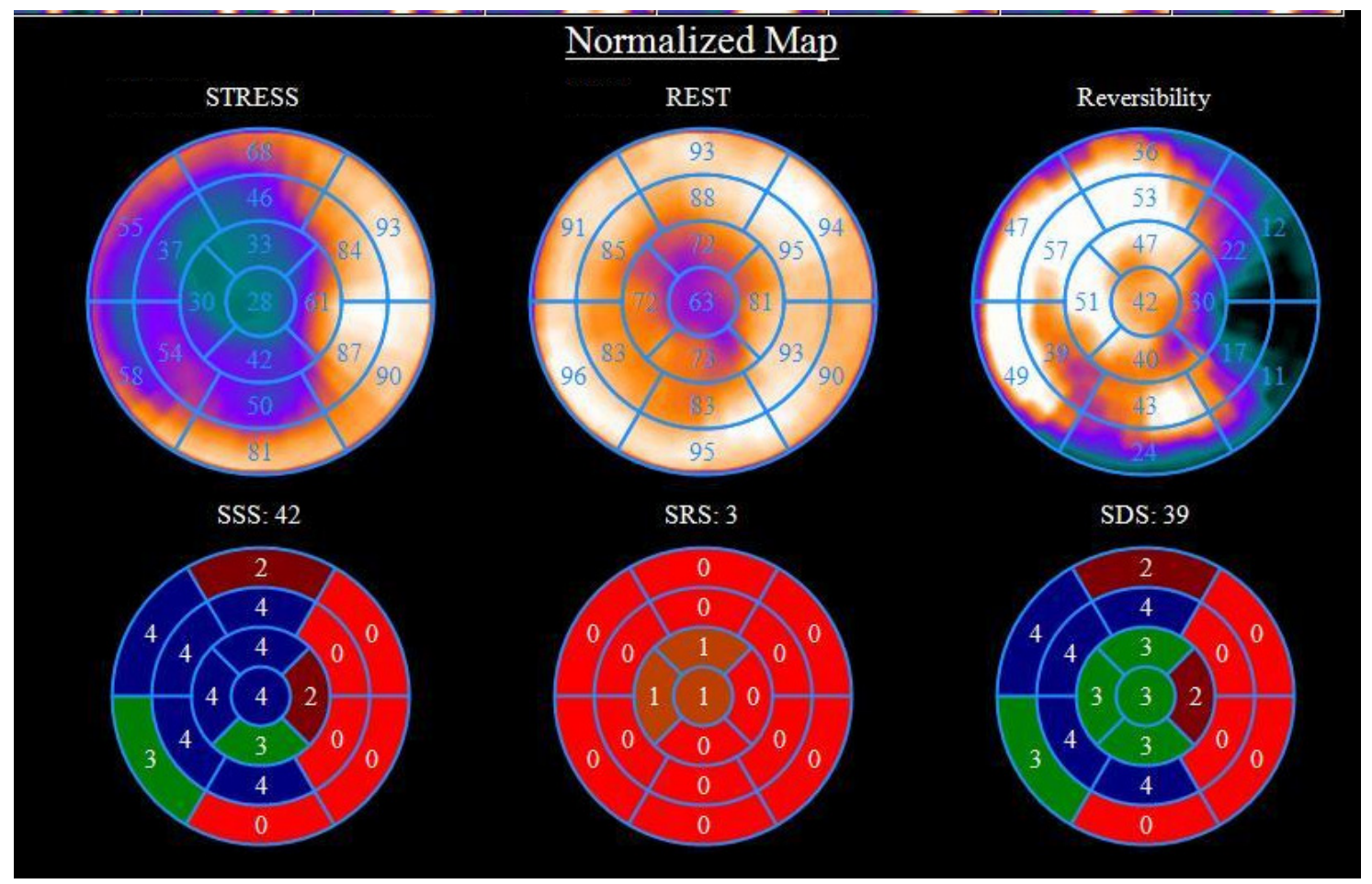

Figure 2.3 - Example of polar-map. There is uptake deficiency in the stress polar-map shown by the blue region which normalizes at rest. The bottom three polar-maps represent the sum-stress, sum-rest, and sum-difference scores with a large region of reversible ischemia in the septal and apical territories of the LV. This pattern is consistent with partial obstruction of the left-anterior-descending coronary artery

LV polar-maps are segmented into regions which are scored depending on their relative tracer uptake generating a semi-quantitative score to represent severity of the uptake defect. A 17-segment model is generally used which uses a scoring scale of 0 to 4 (normal perfusion, mild reduction, moderate reduction, severe reduction, and absence of tracer) [17]. The scores of each segment are summed for rest and stress polar-maps producing global metrics of summed-rest-score (SRS) and summed-stressed-score (SSS). These two metrics are used to determine the severity and extent of the defect. A global 
indicator of reversible ischemia is the summed score differences between rest and stress, known as the summed-difference-score (SDS). In clinical use, a SSS $>3$ signifies the presence of disease while a $S R S>3$ and $S D S>1$ signifies scarred tissue. The 17-segment polar-maps corresponding to these parameters can be seen in Figure 2.3.

The main limitation to this scoring system is that the assumption that the segment with maximum uptake is normally perfused. If a patient has a uniform decrease in perfusion due to disease in multiple coronary vessels then relative uptake image interpretation is likely to under-estimate disease. The polar-map segments are also visually interpreted leading to increased variability in interpretations [18]. Lastly, minimal perfusion deficits

can be difficult to detect when using ${ }^{82} \mathrm{Rb}$ due to this tracers non-linear extraction characteristics. These limitations can be overcome using absolute myocardial blood flow quantification [19].

\subsection{Myocardial perfusion blood flow quantification}

Absolute myocardial blood flow (MBF) quantifies the flow of blood in units of $\mathrm{mL} / \mathrm{min} / \mathrm{g}$ (read as millilitres per minute per gram of tissue). MBF quantification requires dynamic imaging of the tracer as it is injected to the blood stream and distributes to the perfused tissues over several minutes. The ratio between MBF at stress and rest is referred to as myocardial flow reserve (MFR) [20] and has been shown to have better prognostic value than MPI when dealing with patients with suspected cases of CAD [15, 21]. 
While improved diagnostics and patient management has yet to be demonstrated with $M B F$, it has shown promise in the detection of $C A D$ and can add value to the risk assessment and diagnosis of patients [22]. Patients with MFR less than a ratio of 2 are linked with CAD and a MFR ratio higher than 2 are linked with patients with normal myocardial perfusion $[22,23]$, Young healthy individuals are expected to have MFR in the range of 3 to 5 . These MFR ratio values correspond to normal rest MBF values on the order of $0.7 \mathrm{~mL} / \mathrm{min} / \mathrm{g}$ and stress $\mathrm{MBF}$ values $>2 \mathrm{~mL} / \mathrm{min} / \mathrm{g}$, but some variation in thresholds exists in the literature [20, 24, 25].

MBF quantification has been performed with most MPI tracers, but is most common in ${ }^{82} \mathrm{Rb}$ PET due to convenience of use. Using a single list-mode acquisition, dynamic image sequences for MBF quantification can be obtained from the same injection used for traditional MPI therefore requiring no additional time or costs over regular ${ }^{82} \mathrm{Rb} \mathrm{MPI}$.

Dynamic cardiac PET images can image the distribution of tracer from the blood stream into the perfused tissue. Sampling of regions of interest (ROIs) over the dynamic sequence produces time-activity curves (TACS) of the changes in tracer concentration in the anatomical region being sampled. MBF is quantified by analysing the relationship between arterial blood and myocardium TACs. A dynamic image takes images at pre-set time points (e.g. $9 \times 10$ s, $3 \times 30$ s, $1 \times 60$ s, $2 \times 120$ s) as the tracer distributes through the body. Figure 2.4 shows a TAC and the region of activity being sampled. It shows the left ventricle and myocardium continuously being sampled as activity moves from the right ventricle, left ventricle, then during myocardial uptake. 


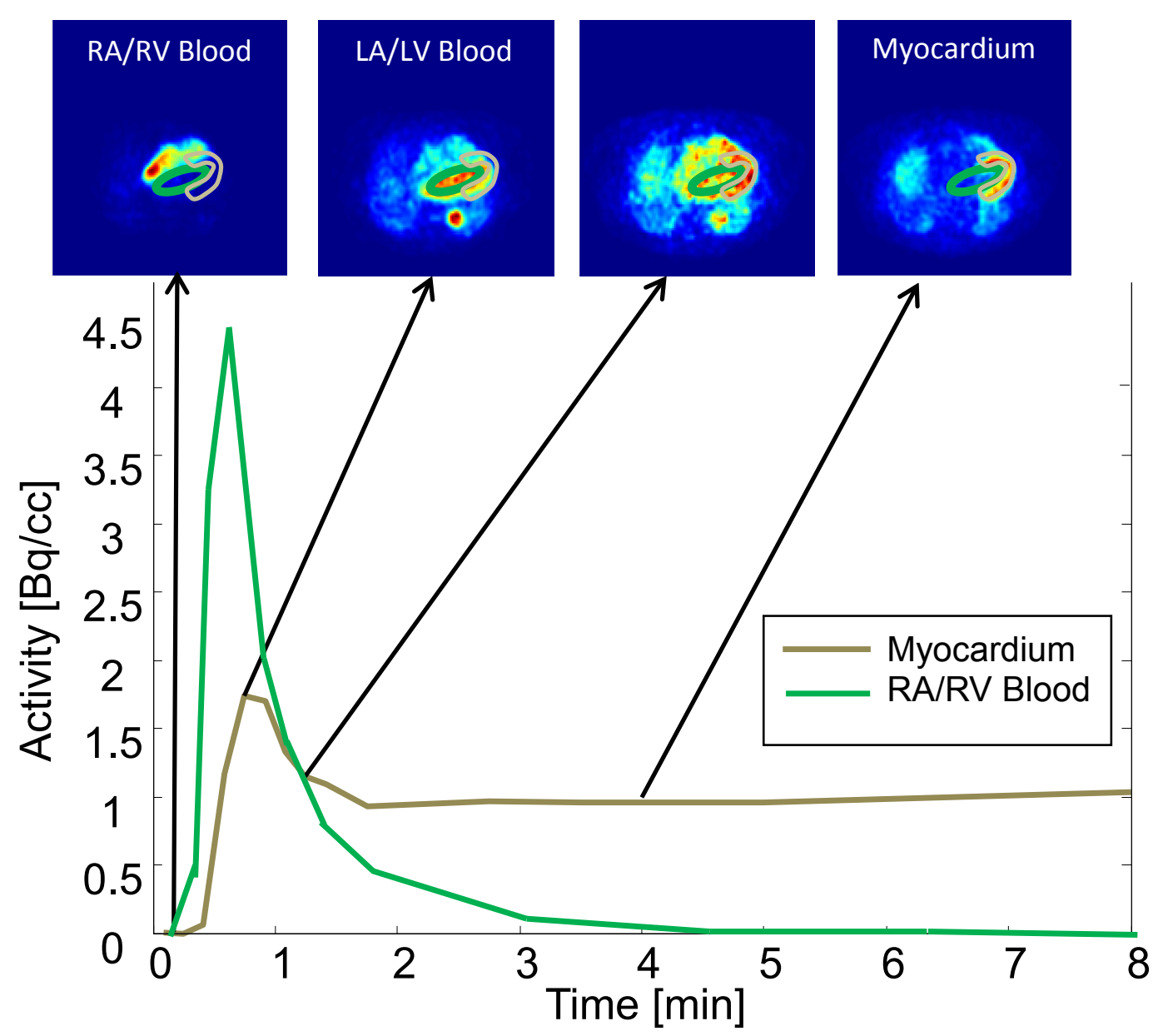

Figure 2.4 - Time-Activity concentration curves (TACs) (bottom) sampled from myocardium and blood region of interest (tan and green respectively) over a dynamic cardiac ${ }^{82} \mathrm{Rb}$ PET image sequence. Representative time frame images are shown on top with each time frame image normalized individually.

To quantify MBF, a compartment model is used to describe the kinetic properties of rubidium uptake by the myocardial tissue [21]. A two-tissue-compartment model describes tracer extraction from the blood to the interstitial space (first compartment) and then into the cellular space (second compartment) in series. Using a two-tissue- 
compartment model (with three rate constants) is acceptable when trying to estimate MBF under noise free condition but when using more realistic noise levels, the model is not reliable [21]. Thus a one-tissue-compartment model (two rate constants) (Figure 2.5 ) is more commonly used with the added benefit of improved processing time [20, 26]. In general, model selection depends on the biochemical properties of the tissue being imaged with regards to the tracer, the image quality, the duration of imaging, and other factors.

The two rate constants in the one-tissue-compartment model are $K_{1}$ and $k_{2}$ (Figure 2.5). $K_{1}$ is measured in $\mathrm{mL} / \mathrm{min} / \mathrm{g}$ and is the uptake rate from the arterial blood to the myocardial tissue. Conversely, $k_{2}$ is the washout rate from the myocardial tissue of the arterial blood in the units of $\mathrm{min}^{-1}$. The one-tissue-compartment model parameters are fitted to reduce errors between the model and measured TACs. The rate of change of rubidium concentrations in the tissue $\mathrm{ROI}$ can be expressed by a simple equation (2-1) where $C_{t}(t)[\mathrm{Bq} / \mathrm{mL}]$ represents the tracers concentration in the myocardial tissue and $C_{a}(t)$ $[\mathrm{Bq} / \mathrm{mL}]$ represents the concentration of tracer in the arterial blood (which perfuses the tissue) with respect to time $(t) . \rho=1.04 \frac{\mathrm{g}}{\mathrm{mL}}$ represents the density of myocardial tissue. 


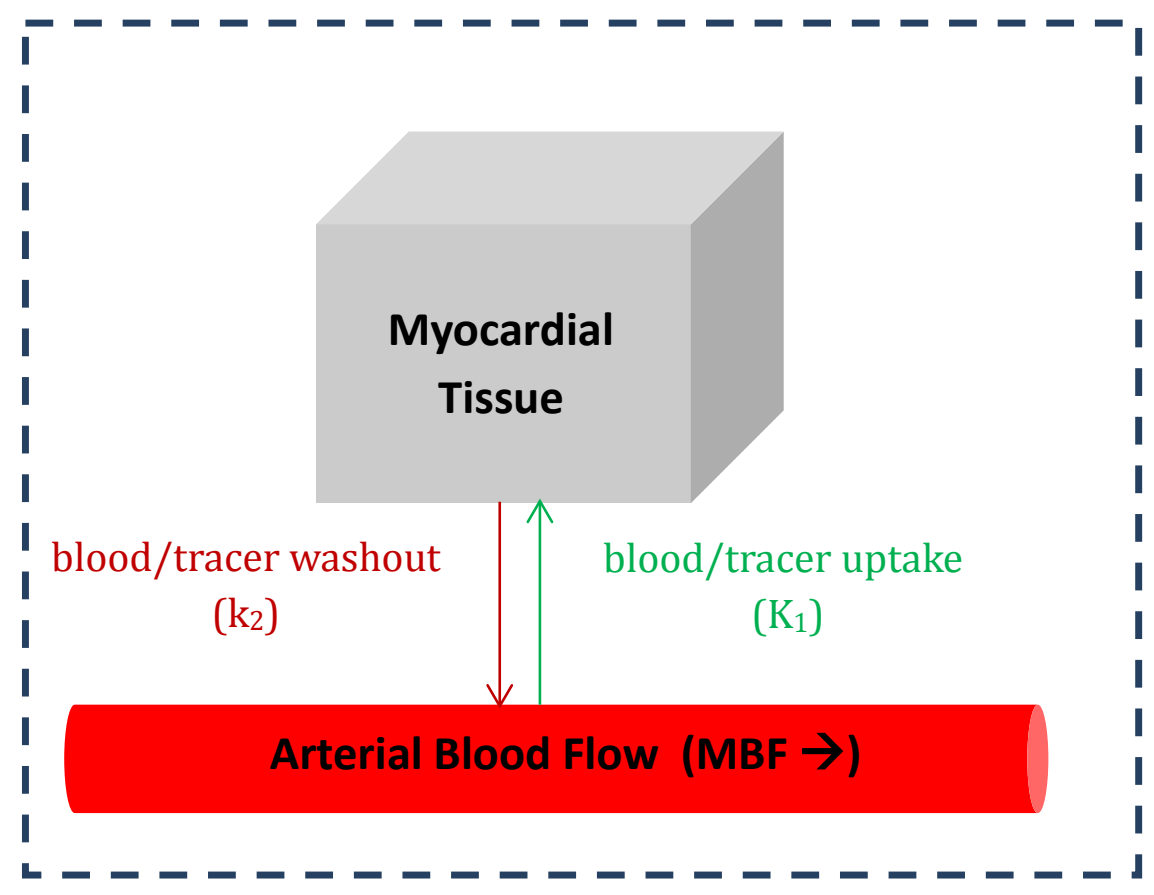

Figure $2.5-{ }^{82} \mathrm{Rb}$ PET one compartmental model used for MBF quantification. Uptake and washout rates of tracers exchange between arterial blood and myocardial tissue are defined by constants $K_{1}[\mathrm{~mL} / \mathrm{min} / \mathrm{g}]$ and $k_{2}\left[\mathrm{~min}^{-1}\right]$.

$$
\frac{d C t(t)}{d t}=\rho \mathrm{K}_{1} \mathrm{Ca}(\mathrm{t})-\mathrm{k}_{2} \mathrm{Ct}(\mathrm{t})
$$

This equation can also be expressed as a convolution kernel $(m(t))$ (equation 2-3):

$$
\begin{aligned}
& C_{t}(t)=C_{a}(t) * m(t) \\
& m(t)=\rho K_{1} \otimes e^{-k_{2} t}
\end{aligned}
$$

In practice, the myocardial ROI encompasses both myocardial tissue and the arterial structures perfusing the tissue with blood as illustrated in Figure 2.5. PET images have a 
limited resolution therefore blood signal from outside the myocardial region may also spillover into the myocardium ROI which increases the amount of blood detected in the ROI. This is in addition to $10-20 \%$ of actual blood volume perfusing the myocardium. Therefore the myocardial TAC, $C_{m}(t)$, is often modelled as a linear combination of both tracer concentration in the myocardial tissue and arterial blood as shown in equation 24. Tissue recovery coefficient (RC) and total blood fraction (TBF) represent the fraction of ROI volume from the myocardial tissue signal and arterial blood respectively. The TBF accounts for the relative blood signal from both the arterial blood volume and spillover signal from the left ventricle (LV) cavity.

$$
C_{m}(t)=T B F \cdot C_{a}(t)+R C \cdot C_{t}(t)
$$

Equations 2-2 and 2-3 can be substituted for $C_{t}(t)$ in equation 2-4 to obtain:

$$
C_{m}(t)=T B F \cdot C_{a}(t)+\rho\left(R C \cdot K_{1}\right) \cdot C_{a}(t) * e^{-k_{2} t}
$$

From equation 2-5 it is clear that $R C$ and $K_{1}$ cannot be uniquely resolved without more information. Some studies have produced calibrated values for RC $[4,27]$ while others automatically estimating $\mathrm{RC}[28,29]$. A more practical (and most commonly used) solution is to assume that the myocardial signal is composed entirely of myocardial tissue and blood signals and therefore RC can be replaced with 1-TBF [31] giving equation (2-6):

$$
C_{m}(t)=T B F \cdot C_{a}(t)+(1-T B F) \cdot \rho \cdot K_{1} \cdot C_{a}(t) * e^{-k_{2} t}
$$


MBF is resolved from $K_{1}$ by correcting for the rate of tracer extraction, which is precomputed for a population. In theory the extraction function accounts for decreasing tracer extraction from the blood into the tissue due to reduced pressure and increased surface area with capillary recruitment in response to vasodilation with stress [31, 32]. Water diffuses freely without the use of active transport from blood into the tissue compartment and therefore $\mathrm{K}_{1}$ directly represents flow. Conversely, most other tracers such as ${ }^{82} \mathrm{Rb},{ }^{13} \mathrm{NH}_{3},{ }^{62} \mathrm{Cu}$-PTSM, and ${ }^{99 \mathrm{~m}} \mathrm{Tc}$-sestamibi are extracted from the blood to tissue using active transport and diffusion and therefore they have a lower extraction fraction at high flow rates [20,31, 32]. Extraction fractions may be represented using the Renkin-Crones capillary model $[31,32]$, and $K_{1}$ is equal to the product between extraction fraction and MBF as shown in equation 2-7:

$$
K_{1}=\left(1-a \cdot e^{-\frac{b}{M B F}}\right) \cdot M B F
$$

In practice the $a$ and $b$ extraction parameters can act as calibration factors compensating for biases associated with the scanning protocol, image reconstruction, ROI definition, tracer kinetic model and quantification processes. They can be determined experimentally [34] or through calibration to known reference standards in a specific populations [21].

\subsection{Motion Artifacts}

Dynamic imaging implicitly assumes that an ROI corresponds to a specific anatomical location in the patient at all time frames. This assumption is violated by the presence of 
motion and may manifest itself as distorted TACs, but may also be inconspicuous. Various types of motions can exist, but for the purpose of this work we make the distinction between rigid and non-rigid motions. Rigid motion assumes that all the activity in the field of view moves together as a solid block without distorting the spatial relationship between any two points in the image. Non-rigid motion does not make this assumption and the image can warp, changing the distances and angles between points in the image. Motion in brain imaging is considered rigid motion since the skull is rigid and firmly encapsulates the brain, while organ motion in the thorax may be non-ridged as the soft tissues can move in relation to one another [35]. Methods have been developed to correct for rigid motion such as using event driven approaches and a multiple acquisition frame methods $[36,37]$. It is more difficult to track non-rigid motion as the direction and magnitude of motion is less predictable from reference landmarks. Several types of non-rigid motions can exist in the context of cardiac imaging:

\subsubsection{Cardiac Motion}

As part of the heart's function of moving blood it contracts and expands every second or faster. Imaging with PET over several seconds to several minutes results in additional blurring of the heart in the image. ECG-gating of the image acquisition is performed as part of MPI protocol to visualize cardiac motion, adding additional diagnostic and prognostic information. Each coincidence event is binned to its corresponding, ECG derived, cardiac phase enabling image reconstruction of each cardiac phase separately, resulting in cardiac-motion-free images. Using list-mode data acquisition, ECG gating can 
be performed using the same MPI or MBF acquisitions. However, in the context of MPI interpretation and MBF quantification, the use cardiac gated images is not desirable as it leads to reduced count statistics and noisy images.

Cardiac motion and the associated motion blur results in increased partial volume loss in the myocardial ROIs, an effect that is compensated for through estimation of $R C$ as part of MBF kinetic modeling. Pre-processing methods for non-rigid motion correction have been proposed. These include gated-PET acquisition during a scan which divides motion cycles into bins at the cost of higher noise level in the images due to a reduced photon count [36, 38]. A motion correction approach uses an expectation-maximization algorithm to simultaneously reconstruct the PET image frames while estimating (and correcting for) cardiac motion [39]. This approach is done during reconstruction therefore it is vendor dependent, is complex requiring more computational power and time, and has not been demonstrated to be sufficiently robust for routine use.

\subsubsection{Respiratory Motion}

Respiratory motion is caused by the natural breathing cycle of the patient, specifically the movement of the diaphragm during inhalation and exhalation of their breath [40]. When dealing with anatomical scanning modalities such as CT it may be practical for the patient to hold their breath as scan times are sufficiently short. When dealing with PET and SPECT where scan times are several minutes long it isn't practical to have the patient hold their breath [41]. Thus respiratory motion can cause degradation of spatial resolution which is most noticeable in cameras with high spatial resolution [41, 42]. 
Respiratory motion can further cause mis-alignment of CT and PET datasets which can produce image artifacts when $\mathrm{CT}$ is used for attention correction (AC), which has been shown to occur in up to $40 \%$ of clinical cardiac PET/CT studies $[6,42]$. A few methods have been proposed by Pourmoghaddas et al. which corrects the PET images using three different maps (respiratory-gated CT map, time- averaged attenuation map and an attenuation map which accounts for the maximum CT-number of every voxel divide by the max respiratory cycle)[42].

Organs proximal to the lungs and diaphragm, including the heart, are most prone to respiratory motion. Even normal breathing can move organs up to $5 \mathrm{~mm}$ in the anteriorposterior, $5 \mathrm{~mm}$ in the left-right and $20 \mathrm{~mm}$ in the superior-inferior directions [43]. Respiratory motion is also a major concern when imaging lung lesions using PET, which could potentially lead to missed finding and underestimation of standard uptake (SUV) or overestimation of the lesion volume [44].

\subsubsection{Heart Organ Motion}

Organ motion refers to incremental (to cardiac, respiratory or patient) motion of individual organs in the thorax such as the liver, pancreas, kidney, and the heart due to anatomical function (e.g. gastro-intestinal) patient relaxation or soft tissue deformation during image acquisition. Organ motion can cause degradation in PET images especially when imaging lesions on these organs. A lesion appears as a blur on the constructed PET image resulting in a decrease in image contrast. This can lead to an underestimation of the lesion uptake or and overestimation of the lesion volume $[45,46]$. 
In the context of cardiac imaging, we are concerned with "cardiac creep" - a mid-scan motion of the heart within the thorax as the organs gradually reposition within the thorax. This motion is associated with patient relaxation during the procedure as well as organ shifting and deformation under the force of gravity after shifting the patient from and upright posture (pre-scan) to supine position during the scan. This motion can be large resulting in inconsistent sampling of the TACs as demonstrated at the bottom of Figure 3.2.

\subsubsection{Patient Motion}

Patient motion is the gross movement of the body during imaging. Patient motion can cause more than $200 \%$ error in measured MBF values [47]. This is often due to the patient being unable or unwilling to hold their position for the duration of the scan. During cardiac PET imaging the patient is usually in supine position with their hands behind their head. This may be a difficult position to endure for certain patients, especially older patients suffering of arthritis. Coughing, fidgeting, and discomfort are common sources of patient motion and while certain measures can be taken to mitigate motion, prevalence of patient motion exceeding $29 \%$ of tests have been reported in large clinical populations [48].

\subsection{Summary}

MBF using PET holds tremendous promise for improving diagnosis, prognosis, and therapy management of CAD patients. As PET technology evolves, however, patient 
motion is increasingly becoming a limiting factor of precision. In this work we explore methods to detect patient motion. 


\section{Agreement between cardiac and fiducial marker motion during dynamic cardiac PET imaging}

\subsection{Introduction}

The work described in this chapter evaluates whether positron-emitting fiducial marker motion is a useful surrogate marker of heart motion in dynamic ${ }^{82} \mathrm{Rb}$ PET image sets.

\subsection{Background}

\subsubsection{Fiducial Marker}

A fiducial marker refers to any type of physical marker that can be attached to the patient (e.g. skin) and then tracked using an external device. Fiducial markers can be used as a surrogate marker of patient and/or organ motion, but may not be tightly correlated with the motion of an organ of interest due to tissue plasticity. While fiducial markers have been extensively used in the context of rigid motion (e.g. head/brain) across different imaging modalities [49-51], relatively little work has been done in the context of non-rigid motion in cardiac applications. Nevertheless some clinical applications do exist. One example application is tracking of respiratory motion to generate respiratory triggers during PET acquisition which are embedded into the raw PET data for further processing. The Real-time Position Management (RPM) system (Varian Medical Systems, Palo Alto, CA) uses two reflective fiducial markers on a lightweight block positioned on the patient's chest. An infrared camera detects respiratory 
motion as the markers move due to the patient breathing $[38,50]$. Reflective fiducial markers have also been used to track pre-reconstruction head motion for neurological PET. This method used an external optical motion tracking camera during imaging. The motion data is aligned with the PET data and inserted into time tags within the list-mode data $[1,2]$. The Positron Emission Tracker (PeTrack) (Carleton University, Ottawa, ON) system can track ${ }^{22} \mathrm{Na}$ fiducial markers ( $<2 \mathrm{~mm}$ motion - phantom study) with high temporal resolution using pre-reconstruction list-mode data (a PET camera generated list of every detected event) [51, 52].

In this work we used a $37 \mathrm{kBq}(1 \mu \mathrm{Ci})$ Sodium-22 fiducial marker affixed non-invasively to a patient's torso during dynamic cardiac PET imaging. The marker appears in the PET image and therefore does not require any external instrumentation for marker tracking. The marker appears as a roughly $6 \times 6 \times 6$ pixel volume in the dynamic PET image (Figure 3.1). It is important to note that the center of the marker has higher pixel intensity and it decreases radially outward due to the limited spatial resolution of PET. Figure 3.2 shows an example of fiducial marker motion (top), heart motion (bottom) and the corresponding discontinuity in the respective time-activity curves. Since the marker activity is long-lived (2.6 year half-life) the marker intensity appears to exponentially grow over the course of the decay-corrected (76 s half-life of ${ }^{82} \mathrm{Rb}$ ) dynamic image sequence. 


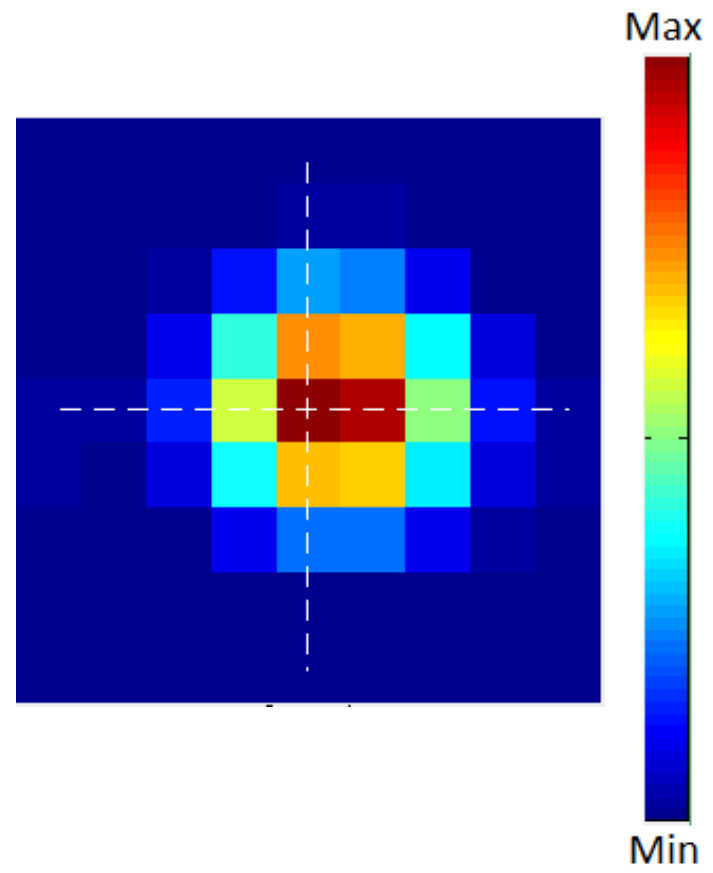

Figure 3.1 - Example of fiducial marker in a PET image frame. The intensity of the pixels decreases moving radially outward from the center of the marker due to limited spatial resolution of PET images. 

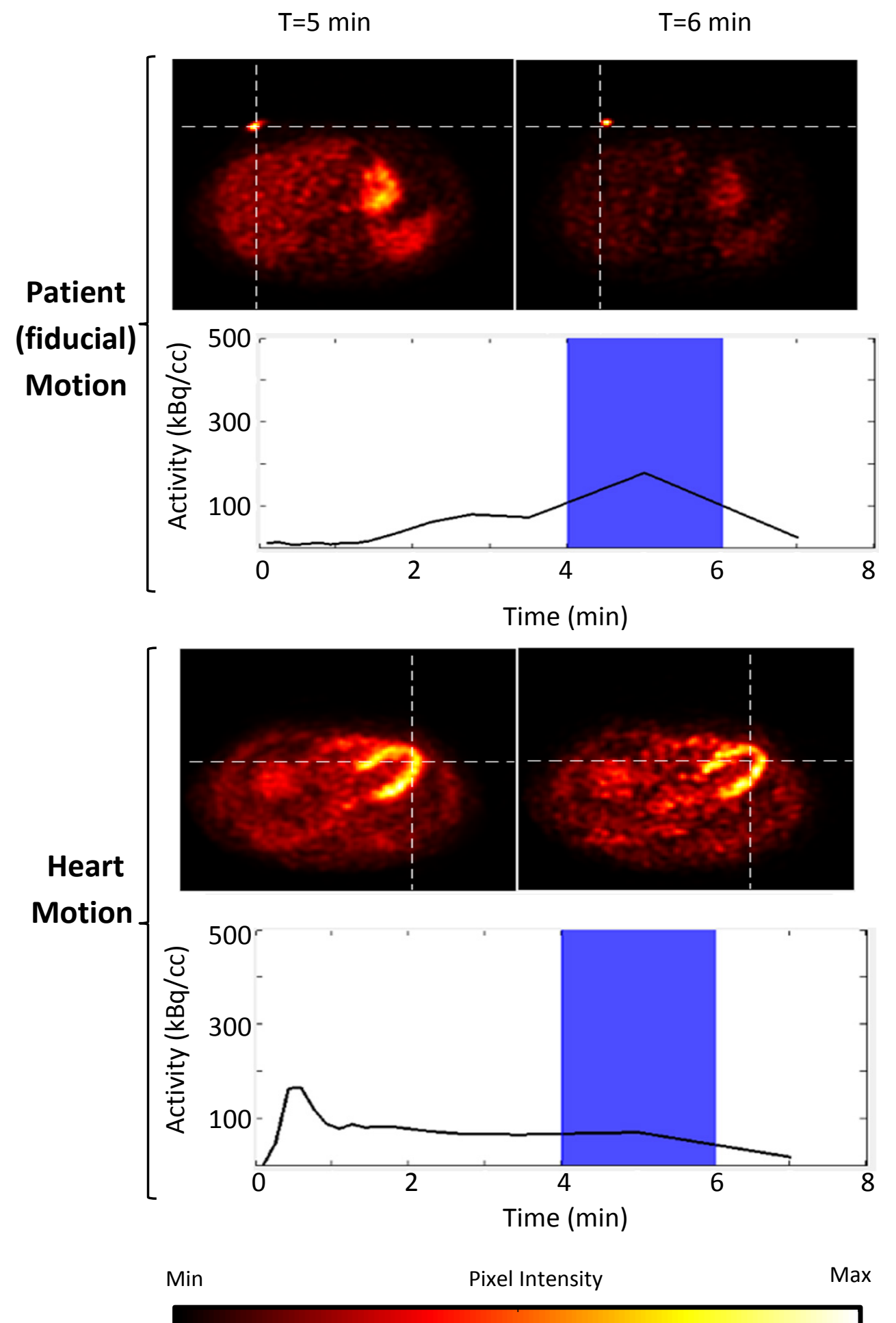

Figure 3.2 - Top: Fiducial marker motion between two frames and resulting distortion of time-activity curve between 5-6 mins. Bottom: Heart organ motion in the same 
direction and time as the fiducial marker motion. The cross-hair was not moved between frames in both images.

We sought to determine the degree by which fiducial marker motion is correlated with heart motion in order to elucidate whether fiducial markers may be used as surrogates to detect patient motion.

\subsection{Methods}

\subsubsection{Patient Cohort}

Sixty five (65) clinical patients that had undergone cardiac rest-stress dynamic ${ }^{82} \mathrm{Rb}$ PET imaging at the University of Ottawa Heart Institute, National Cardiac PET Centre were arbitrarily selected between September 2012-August 2013 (date range of scans) for this study. All patients were instructed not to consume beta-blockers for 24 hours, caffeine for 12 hours and to refrain from food for 4 hours. All patients gave consent as approved by the Ottawa Heart Institute Research Ethics Board as part of the Rubidium-ARMI (NCT01128023) research protocol.

Imaging was performed according to the standard clinical protocol (seen in Figure 3.4) for imaging of patients using a state-of-the art GE Discovery 690 VCT PET-CT scanner

(Waukesha, WI). A $37 \mathrm{MBq}{ }^{22} \mathrm{Na}$ fiducial marker (1 mm diameter) was affixed to the patient lower anterior-right rib with a saline bag covering to induce adequate scatter for the marker to not interfere with the camera's scatter correction algorithm (CT image shown in Figure 3.3). 


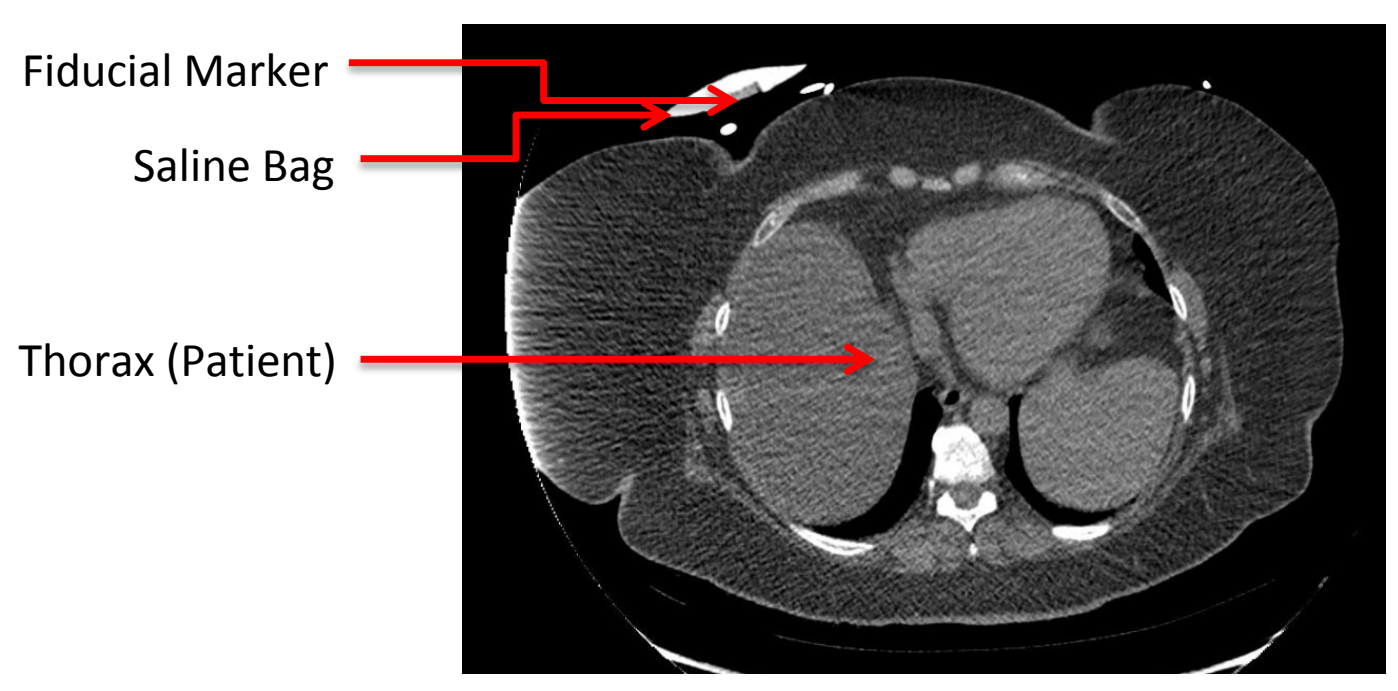

Figure 3.3 - Computed Tomography slice of patient in the transverse plane. The fiducial marker can be seen on the outside of the patient's chest. A saline bag was placed over the marker to avoid interference with the scatter correction algorithm. The marker was placed away from the heart to avoid interference with clinical interpretation.

A low-dose $0.5 \mathrm{mSv}$ normal-end-expiration CT scan lasting 1.5 s was performed first for attenuation correction with the patient at resting state. Patients were then injected with ${ }^{82} \mathrm{Rb}$ tracer (10 MBq/kg over $30 \mathrm{~s}$ interval) using a custom 'square wave' infusion system (RubyFill ${ }^{\mathrm{TM}}$, Jubilant Draxlmage, Kirkland, QC) [55] and PET list-mode image acquisition ( 8 minutes) was manually started when the true count-rate exceeded 10 kcps (kilo-counts per second). Dynamic images were reconstructed using filtered back projection (12 mm Hann filter) with $128 \times 128 \times 47$ voxels of size $3.125 \times 3.125 \times 3.270 \mathrm{~mm}$ 
and an isotropic spatial resolution of $10 \mathrm{~mm}$ FWHM into 15 time frames. Table 3.1 describes the time length of each of the 15 times frames.

Table 3.1 - Time frame numbers and the corresponding time frame start time and length time.

\begin{tabular}{|c|c|c|}
\hline $\begin{array}{c}\text { Time Frame } \\
\text { Number }\end{array}$ & $\begin{array}{c}\text { Time Frame } \\
\text { Start Time (s) }\end{array}$ & $\begin{array}{c}\text { Time Frame } \\
\text { Length (s) }\end{array}$ \\
\hline 1 & 0 & 10 \\
\hline 2 & 10 & 10 \\
\hline 3 & 20 & 10 \\
\hline 4 & 30 & 10 \\
\hline 5 & 40 & 10 \\
\hline 6 & 50 & 10 \\
\hline 7 & 60 & 10 \\
\hline 8 & 70 & 10 \\
\hline 9 & 80 & 10 \\
\hline 10 & 90 & 30 \\
\hline 11 & 120 & 30 \\
\hline 12 & 150 & 30 \\
\hline 13 & 180 & 60 \\
\hline 14 & 240 & 120 \\
\hline 15 & 360 & 120 \\
\hline
\end{tabular}

A second PET acquisition followed in the same fashion as the rest imaging, but with the patient stressed using dipyridamole, a pharmacologic vasodilator, which was intravenously administered over 5 minutes at a rate of $0.14 \mathrm{mg} / \mathrm{kg} / \mathrm{min}$. Three minutes into the administration of dipyridamole the patient was injected with ${ }^{82} \mathrm{Rb}$ a second time and image acquisition followed as at rest-state. A second CT scan was then completed for attenuation correction of the stress image. The standard clinical protocol is summarized in Figure 3.4 
Table 3.2 summarizes patient information for the cohort.

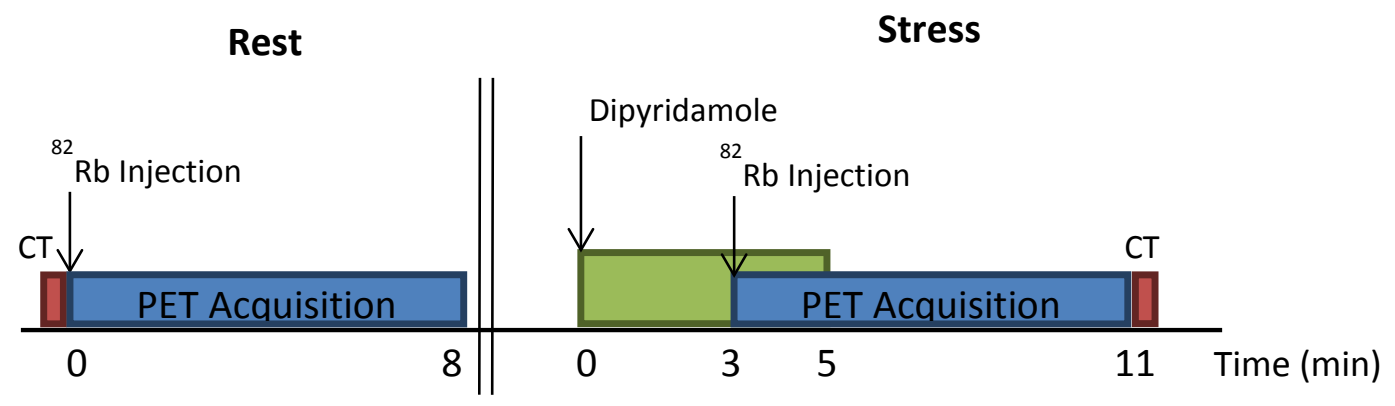

Figure 3.4 - Clinical rest-stress ${ }^{82} \mathrm{Rb}$ PET/CT protocol used for methods

Table 3.2 - Patient information for patient cohort used

\begin{tabular}{|c|c|}
\hline Patients & $\begin{array}{ll}\text { Combined: } & 65 \\
\text { Male: } & 36 \\
\text { Female: } & 29\end{array}$ \\
\hline Mean Age (years) & $67 \pm 12$ \\
\hline Mean Weight (kg) & $87 \pm 18$ \\
\hline Mean Height (m) & $1.68 \pm 0.10$ \\
\hline
\end{tabular}

\subsubsection{Visual Image Analysis}

To characterize heart motion, for correction, the time frame and direction of the motion needs to be accurately resolved. Dynamic images were visually inspected for the presence of both significant heart and patient (i.e. fiducial marker) motion by comparing all neighbouring image frame pairs in the dynamic sequence. The 130 images (rest and stress) were visually evaluated using an in-house software program (FlowQuant $\mathbb{C}$, Ottawa, ON) $[14,19]$ for heart and patient motion in a $128 \times 128 \times 47$ pixel volume of 
interest (VOI) with 15 time frames. Motion amplitude was classified as no-motion or significant motion ( $\geq 2$ pixels). Significant motion events ( $\geq 2$ pixels) were easily identifiable compared to mild motion ( $<2$ pixels), in part due to the large file of view. Patient and heart motion could rarely be viewed in the same slices and were thus assessed separately.

The presence/absence of motion, time frame and approximate direction of heart and patient motions was recorded. Each direction of motion was classified as X left $(+), X$

right (-), $\mathrm{Y}$ anterior $(+), \mathrm{Y}$ posterior (-), $\mathrm{Z}$ superior $(+)$, $\mathrm{Z}$ inferior (-). Multiple motion events could be recorded per dynamic image. The prevalence and concordance of patient and heart motion was analyzed from this data. Furthermore, the sensitivity and specificity of using fiducial markers as a surrogate marker for heart motion was determined.

\subsection{Results}

Either patient or heart motion was present in $40(30.8 \%)$ of the 130 dynamic PET images. Patient motion was detected in $37(28.5 \%)$ images and heart motion was detected in $27(20.8 \%)$ images. $24(18.5 \%)$ images had both patient and heart motion. Among all images $(n=130)$, patient and heart motion agreement rate was $88 \%$ (cases without motion and with both heart and fiducial marker motions present). The sensitivity and specificity to predict the presence of heart motion from patient motion was found to be $89 \%$ and $87 \%$ respectively. See Table 3.3 for visual assessment results. 
Table 3.3 - Presence of Patient and Heart motion in Patient Dynamic PET images $(n=130)$. Significant motion was defined as $\geq 2$ pixels.

\begin{tabular}{|c|c|c|}
\hline & Patient Motion & No-Patient Motion \\
\hline Heart motion & $18.5 \%$ & $2.30 \%$ \\
\hline No-Heart motion & $10.0 \%$ & $69.2 \%$ \\
\hline
\end{tabular}

Of the 24 images with patient and heart motion, $70.8 \%$ of these images had motion in the same time frame (1-15) and the same direction ( $x, y$ or $z) .12 .5 \%$ of the images with motion were in different time frames and $16.7 \%$ of the images had motion in different directions (See Table 3.4).

Table 3.4 - Agreement between motion time frame and primary direction in cases with both patient and heart motion $(n=24)$

\begin{tabular}{|c|c|c|c|}
\hline $\begin{array}{c}\text { Same } \\
\text { time and direction }\end{array}$ & $\begin{array}{c}\text { Different } \\
\text { time frame }\end{array}$ & $\begin{array}{c}\text { Different } \\
\text { primary direction }\end{array}$ & Total \\
\hline $70.8 \%$ & $12.5 \%$ & $16.7 \%$ & $100 \%$ \\
\hline
\end{tabular}

\subsection{Discussion}

This work investigated the relationship between heart motion and motion of skin bound fiducial markers. Both the heart and the fiducial markers were visible in the PET volume images, with the fiducial marker assumed as a surrogate marker of patient motion. Automatic tracking of heart motion in a dynamic cardiac image sequence can be difficult due to the changing tracer distribution from blood to perfused tissues while a fiducial marker has a relatively constant distribution across all time frames providing a more stable, isolated and highly visible signal to track (Figure 3.1). 
Post-reconstruction detection of fiducial marker motion is an attractive alternative to pre-reconstruction fiducial marker motion detection which requires special equipment such as an infrared camera $[38,50]$ or an optical motion tracking camera $[1,2]$. The use of external motion tracking systems not only adds costs, but also significant complexity associated with motion pre-processing and non-trivial correction of the prereconstructed PET measurements. Lastly, directly using list-mode data is camera/vendor specific, whereas a post-reconstruction algorithm is vendor independent. The agreement between presences of motions in the entire dynamic study was $88 \%$ for flagging cases with heart motion based on analysis of fiducial marker motion. In the subset of images which had patient and heart motion, $70.8 \%$ had motion in the same time frame and same direction while $16.7 \%$ had motion in different time frames and $12.5 \%$ in different directions. Based on these results we concluded that tracking of a single fiducial marker is not adequate for characterizing motion and therefore guiding motion correction, but that fiducial marker motion can be a good predictor of the presence of heart motion.

\subsubsection{Motion timing}

Two classifications of motion timing were visually inspected in the images. Motion can manifest itself as inter-frame motion in which a mis-alignment exists between subsequent time frames, or as intra-frame motion in which the image is blurred due to a shift in patient positioning within a time frame. Inter- and intra-frame motions will typically coexist and depend on the time and nature of motion with respect to the time 
frame intervals [56]. Therefore both types of motion must be detected if a robust automatic motion detection algorithm is developed.

\subsubsection{Multiple fiducial markers}

Multiple markers have been used to accurately characterize motion of the brain in all six directions [57] which could improve the characterization of heart motion and therefore guide motion correction. Patient rotations and translations in multiple directions cannot be detected with a single marker, requiring multiple markers at different anatomical locations. We speculate that the additional information would contribute to detection of a wider range of motion and may enable better characterisation of heart motion.

\subsubsection{Limitations}

Visual interpretation is vulnerable to viewer bias and errors, and is limited to qualitative assessment. Nevertheless, it is not possible to induce motion in clinical studies and another (external) reference was not available to us for this study. We did not observe mild motion which most likely would not be clinically significant in causing motion artifacts but more work and analysis must be done to verify these assumptions. Mild motion ( $<2$ pixels in magnitude by our definition) was difficult to view in the full VOI $(128 \times 128 \times 47$ pixel volume) and inclusion in the analysis could increase the error rate in the visual assessment of motion. Motion less than 2 pixels in magnitude corresponds to $<6.3 \mathrm{~mm}$ which is less than image spatial resolution $(\sim 10 \mathrm{~mm})$. Therefore we do not expect motion of this magnitude to have clinically significant impact on MBF estimates [47]. Since this chapter evaluated the use of fiducial markers as a surrogate marker of 
heart motion in a clinical setting, we felt that it was important to focus on clinically significant motion and minimizing visual assessment errors. Therefore, we only included significant motion events ( $\geq 2$ pixels) and neglected mild motion events.

\subsection{Conclusion}

A single fiducial marker is a modest surrogate marker of heart motion time and direction, making it inadequate in guiding motion correction. However, fiducial markers have adequate sensitivity and specificity as predictors of presence of heart motion in dynamic image acquisitions, making it a suitable method for flagging motion artifacts. The next chapter describes two automatic detection methods for fiducial marker in dynamic PET cardiac images. 


\section{Automatic Detection of Patient Motion Using Fiducial Markers in Dynamic Cardiac PET Images}

\subsection{Introduction}

This chapter describes automatic methods to detect inter- and intra-frame fiducial marker motion in dynamic cardiac PET images. Numerical-phantom based simulated images with known simulated motion are used to validate the algorithms. Clinical data are used to characterize the accuracy of these algorithms for flagging the presence of motion with visual interpretation serving as a gold standard.

\subsection{Background}

Motion can manifest itself as inter-frame motion in which a mis-alignment exists between subsequent time frames, or as intra-frame motion in which the image is blurred due to a shift in patient positioning within a time frame. Inter- and intra-frame motions typically coexist and depend on the time and nature of motion with respect to the time frame intervals [56].

While many technologies exist for tracking fiducial markers, we focused on positronemitting markers which can be imaged with PET and therefore are inherently integrated into the reconstructed images for vendor independent, post-reconstruction analysis. Of particular interest to our group is incorporation of motion detection into clinical MBF quantification software for quality control. 


\subsection{Method}

To test whether fiducial marker motion can be automatically detected two algorithms were designed, tested, analyzed and optimized for performance using statistical analysis. A series of simulated and patient dynamic images were processed as a baseline for the algorithms performance. The workflow for the inter- and intra-frame algorithms is shown in Figure 4.1 and Figure 4.2 respectively.

\section{Inter-frame Motion Algorithm Workflow}

Design
Requirements
- Post-
reconstruction
- Low-cost
- Fast processing
-Accurately
detect motion
between frames

Implementation
-Isolate fiducial
marker
-Image
conditioning
-Inter-frame
motion detection

Testing
-Noiseless NCAT
models with
simulated
motion
-PET Patient
images with
visual assesment
of motion

Analysis and
Results
-ROC statistical
analysis
-Optimal D
threshold value
-Accuracy,
Sensitivity,
Specificity

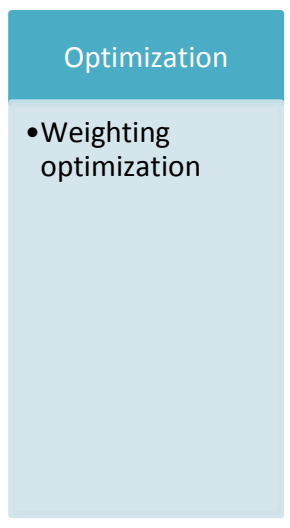

Figure 4.1 - Workflow for design, implementation, testing and analysis of the interframe motion detection algorithm 


\section{Intra-frame Motion Algorithm Workflow}

Design
Requirements
- Post-
reconstruction
- Low-cost
- Fast processing
-Accurately
detect motion
blur within a
frame
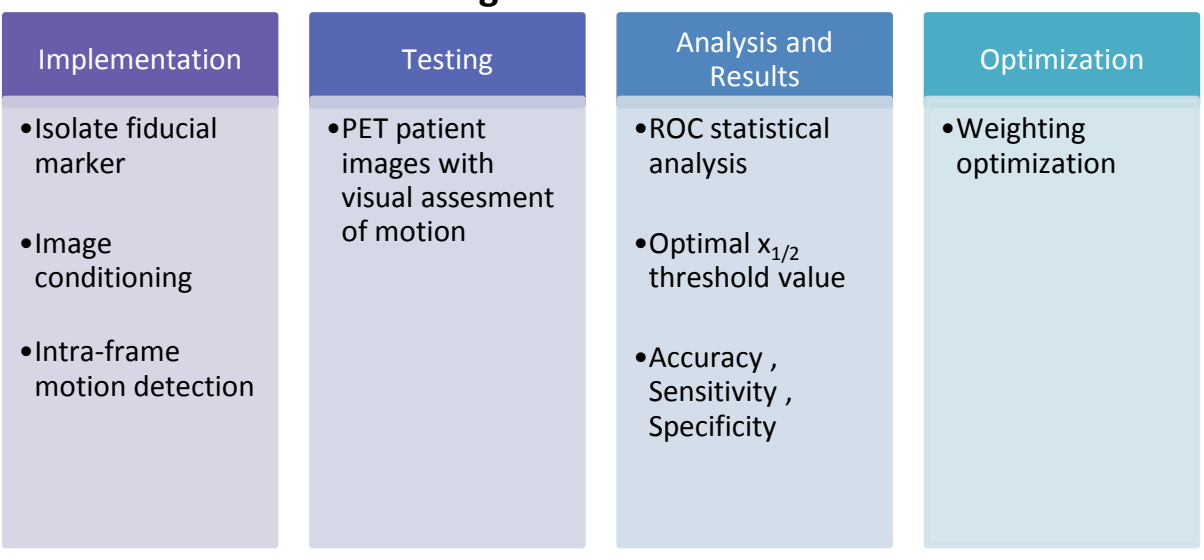

Figure 4.2 - Workflow for design, implementation, testing and analysis of the intraframe motion detection algorithm

The specifics of the methodology are described in the following sections.

\subsubsection{Inter-frame Motion Simulations}

Fifty four noise-free non-uniform rational b-spline (NURBS)-based cardiac-torso (NCAT)(Johns Hopkins University, Baltimore, MD) numerical-phantom based dynamic image sequences were generated [56-58]. NCAT is a computer software package which is widely used to construct customizable high resolution dynamic PET numericalphantom based simulation models of the human physiology and anatomy. This program allows the user to assign activity to major organs. The models can also simulate eight different components of the heart which include the right and left, ventricle and atrial, blood-pool and wall activities. The model dimensions were $40 \mathrm{~cm} \times 40 \mathrm{~cm} \times 19.7 \mathrm{~cm}$ with a cubic voxel size of $3.125 \mathrm{~mm}$ and a transverse sampling grid of $128 \times 128$ pixels in 63 slices. The dynamic image sequence consisted of 15 time frames (Table 3.1) corresponding to 10 minutes of data. Organ time-activity curves were derived from a 
previous clinical ${ }^{82} \mathrm{Rb}$ dosimetry study in human patients [61] using the same blood TAC for the left and right chambers of the heart. A fiducial marker was simulated as a 9.375 $\mathrm{mm}$ diameter sphere positioned on the right-anterior lower thorax (roughly in plane with the upper liver and inferior heart wall). The marker activity was exponentially grown with time to simulate a constant activity source in an ${ }^{82} \mathrm{Rb}$ decay-corrected image sequence. An example of two slices showing the fiducial marker and myocardium can be seen in Figure 4.3.

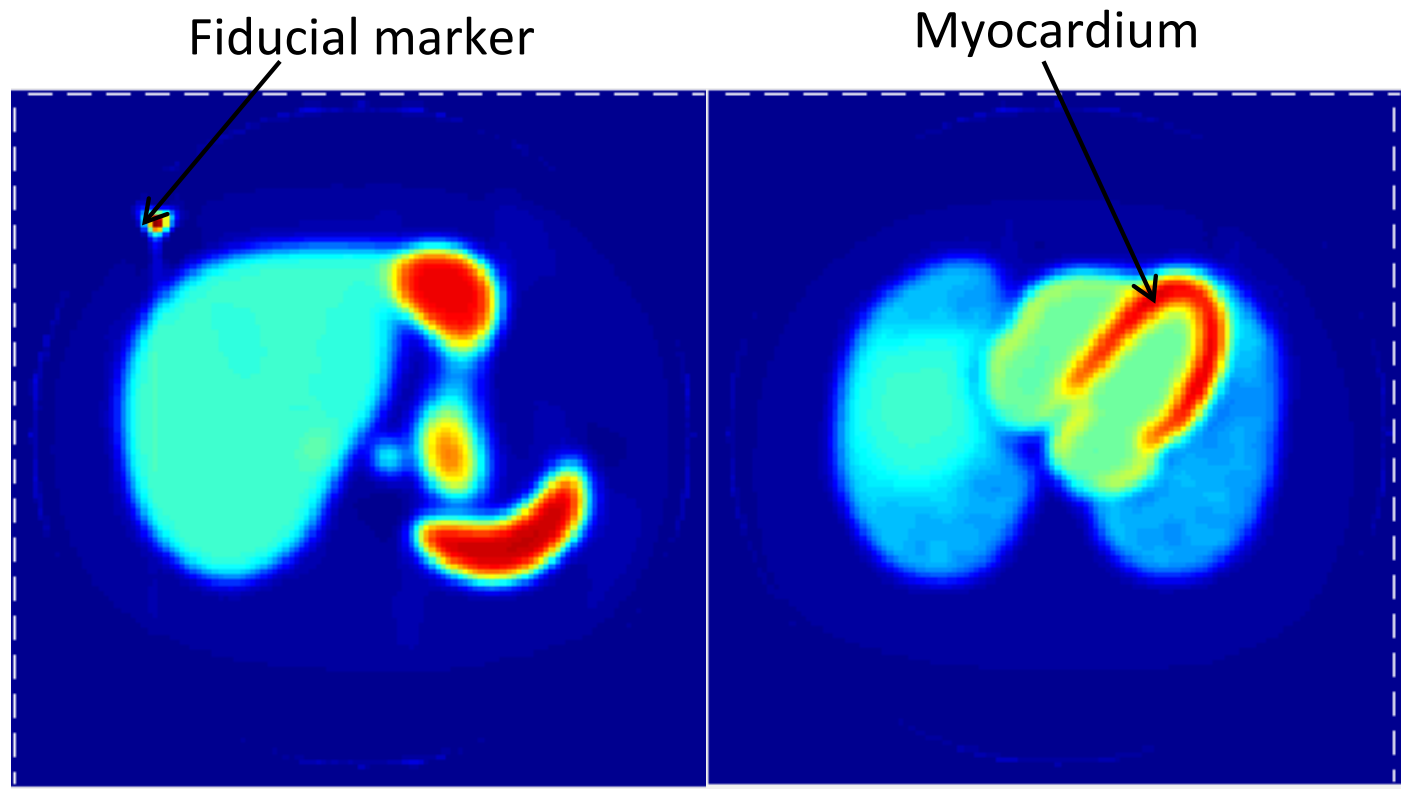

Min

Pixel Intensity

$\operatorname{Max}$

Figure 4.3 - Noise-free simulation model. The left image shows the transverse slice which contains the fiducial marker. The right image shows the transverse slice which contains the myocardium in the same time frame. The red areas are high activity regions and the blue areas are low-activity regions 
Inter-frame (between time frames) motion was simulated as a single event in a dynamic image sequence at $30,60,80,90,120,150,180,240$, or 360 second time points with $\pm 9.375 \mathrm{~mm}$ (referred to as $1 \mathrm{~cm}$ ) of body motion in 6 directions ( $+x$ right, $-x$ left, $+y$ posterior, $-\mathrm{y}$ anterior, $+\mathrm{z}$ superior, or $-\mathrm{z}$ inferior). Once motion was introduced it was not corrected for all the subsequent time frames, thus simulating a single motion event in each model.

In clinical practice the CT is aligned with the last frame, thus all frames preceding the motion event would have CT attenuation correction (CTAC) mis-registration artifacts. However, attenuation was not simulated in this dataset so as to focus on motion alone.

Images were blurred using a 3D Gaussian filter $(8.5 \mathrm{~mm}$ full width at half maximum (FWHM)) to account for the intrinsic scanner resolution and the positron range of ${ }^{82} \mathrm{Rb}$. NCAT images were then forward projected (128 Projections) using a radon transfer function and then the sinogram was reconstructed using filtered back projection (FBP). The reconstructed images were then filtered using a 3D Gaussian filter to obtain 2 and $10 \mathrm{~mm}$ FWHM spatial resolution (referred to as high and low resolution respectively). No noise was added to the data at any stage. An example of the simulation model in the transverse plane can be seen in Figure 4.3. 


\subsubsection{Patient Cohort}

The same patient cohort as described in section 3.3.1 was used for this experiment following the same protocols for image acquisition. The protocol is summarized in Figure 3.4. Table 3.2 summarizes patient information for the cohort.

\subsubsection{Visual Image Analysis}

The 130 patient images (rest and stress) were cropped to a 9x9x9 VOI containing the entire fiducial marker and were visually (re-)evaluated using an in-house software program (FlowQuant $\complement$, Ottawa, ON) $[14,19]$ for the presence of any patient motion (mild and/or significant). Patient motion was seen if there was a movement in the fiducial marker in the dynamic PET image. The time frame of patient motion was recorded on a frame-by-frame basis for inter-frame (marker motion between frames) and intra-frame motion (blurred motion within a time frame).

\subsubsection{Automatic Fiducial Marker Motion Detection}

Localization of the marker in the late time frame images was trivial as the marker activity concentration (image intensity) was much higher than that of the patient as demonstrated in Figure 4.4. Therefore, the maximum intensity pixel was assumed to correspond to the center of the marker. 


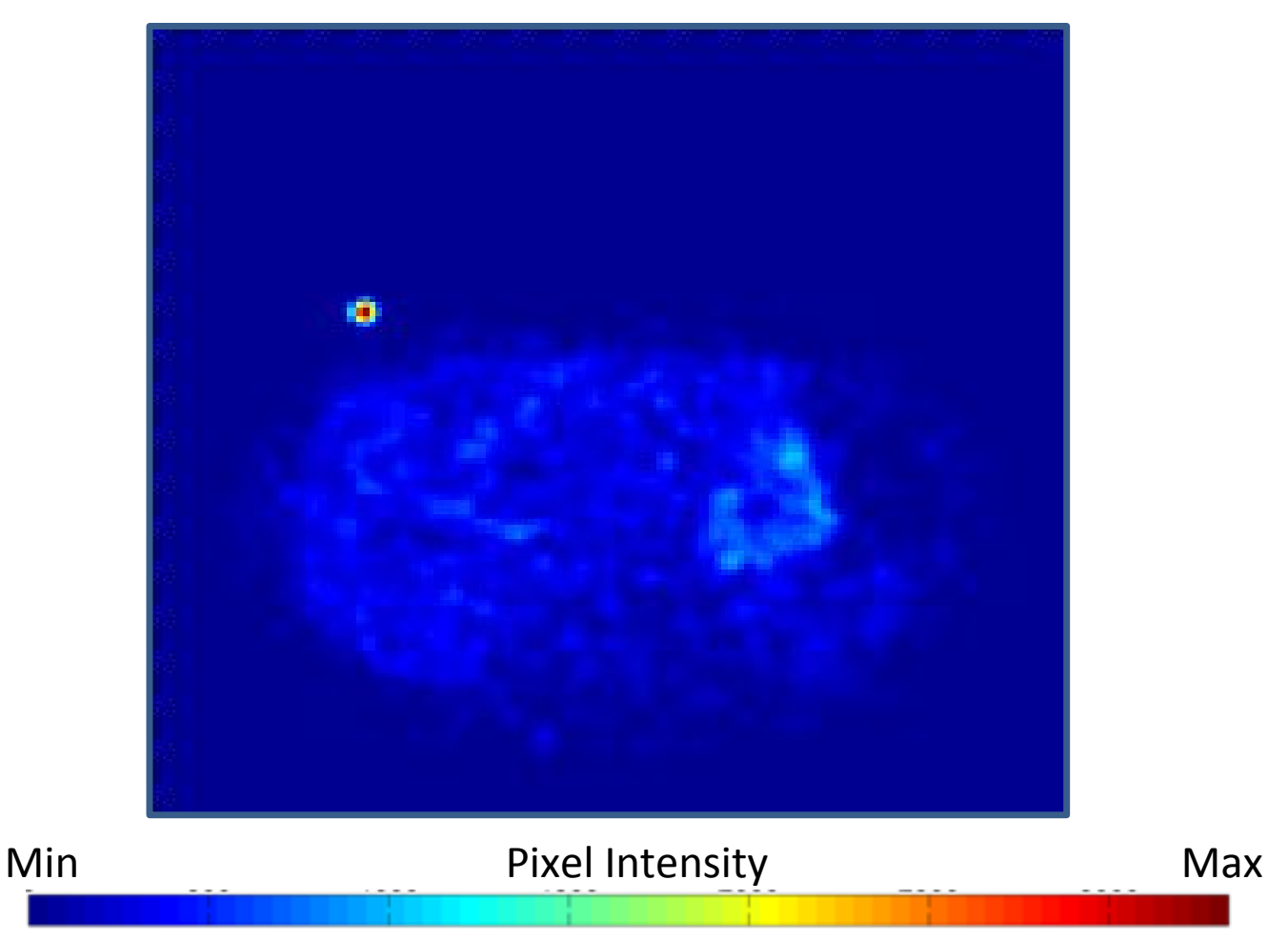

Figure 4.4 - Fiducial marker contains most intense pixels in time frame 15. Therefore localization of marker occurs in this last time frame.

\subsubsection{Image Conditioning}

A 9x9x9 pixel volume of interest (VOI) around the fiducial marker (Figure 4.5) was isolated and processed to reduce the effects of interference from neighbouring patient activity and noise. The 3D pixel intensity plot of the transverse plane in Figure 4.5 is shown in Figure 4.6 with the fiducial marker at the center and patient activity spilling in to the marker in the left-hand corner. 

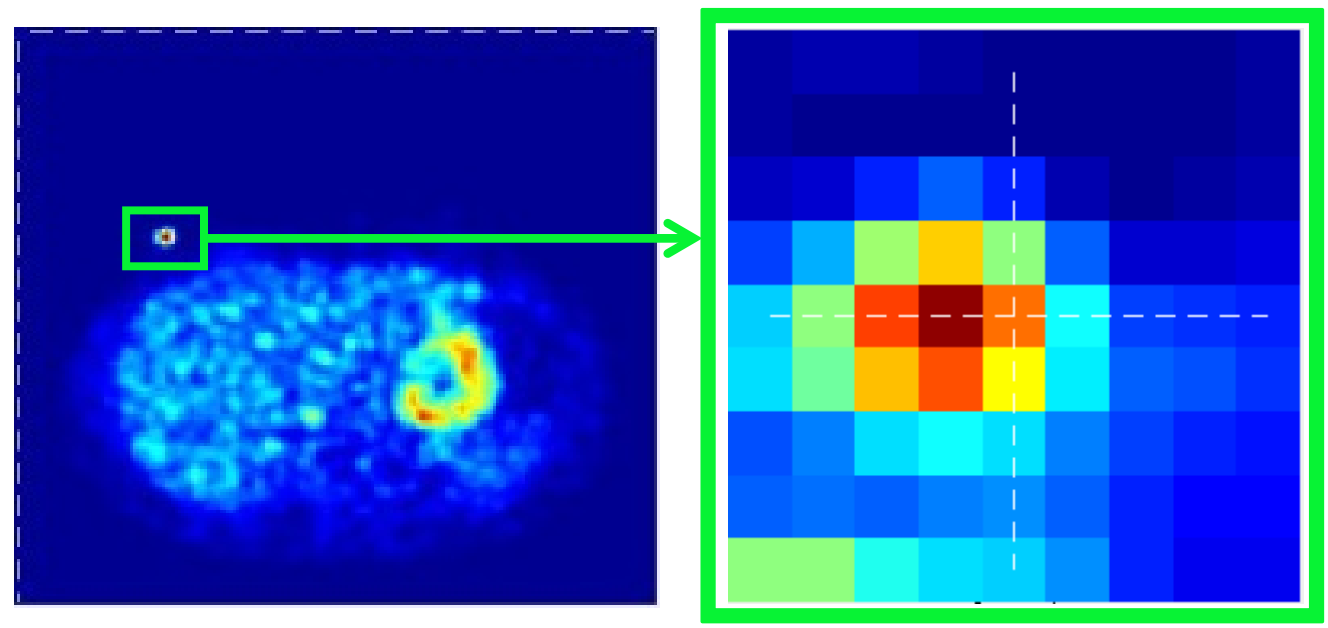

Min

Pixel Intensity

$\operatorname{Max}$

Figure 4.5 - Global (left) and local ROI (9×9 pixels) around the fiducial marker demonstrating background activity spilling in from the patient. This marker is taken from time frame 14 of an image with motion present. Spill-in activity from the patient is also evident.

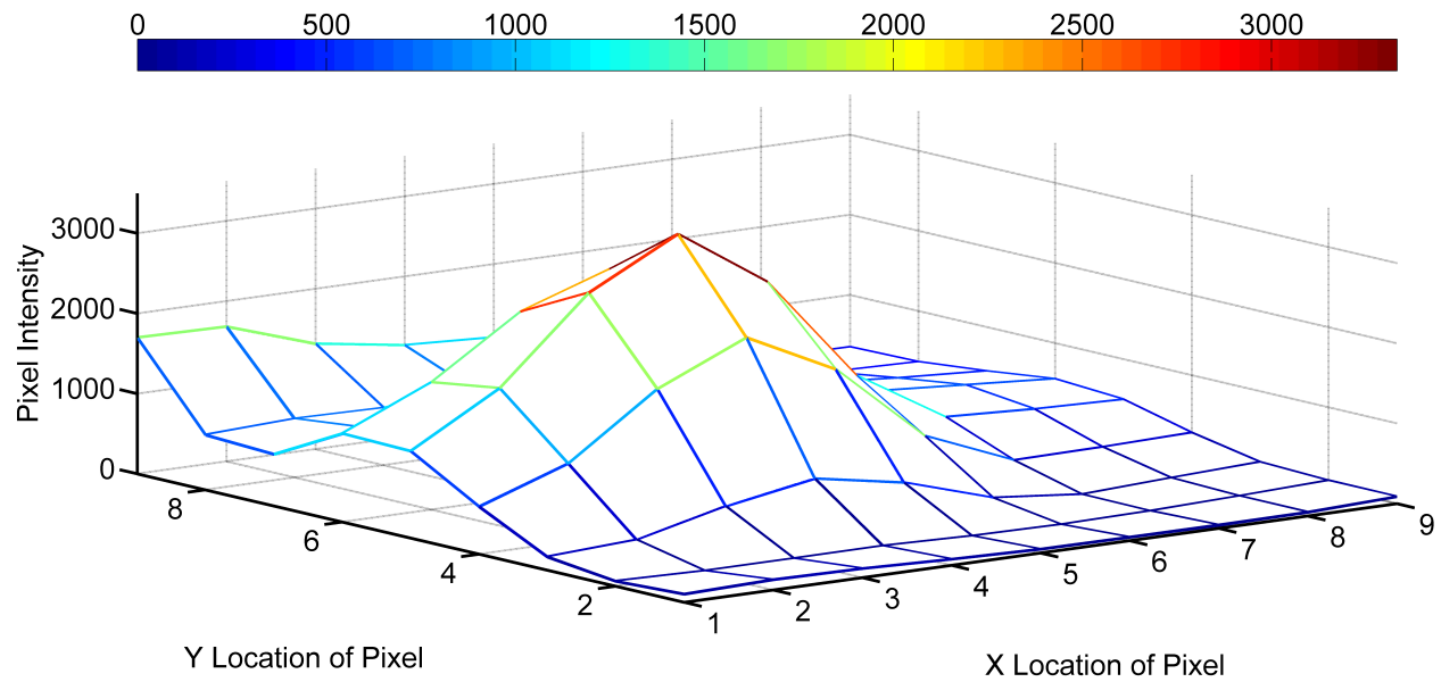

Figure 4.6 - 3D mesh-plot representing the pixel intensities of a fiducial marker in a 9x9 pixel volume in the transverse plane with spill-in activity from the patient (left corner). 
To improve the background to marker contrast, pixel intensities below a threshold value were set to zero. The threshold value (4-1) was set as $50 \%$ level between the minimum background and the peak pixel intensities in the VOI. The resulting thresholded transverse plane of Figure 4.6 demonstrated in Figure 4.7.

Threshold $=\frac{\text { Peak+Background }}{2}$

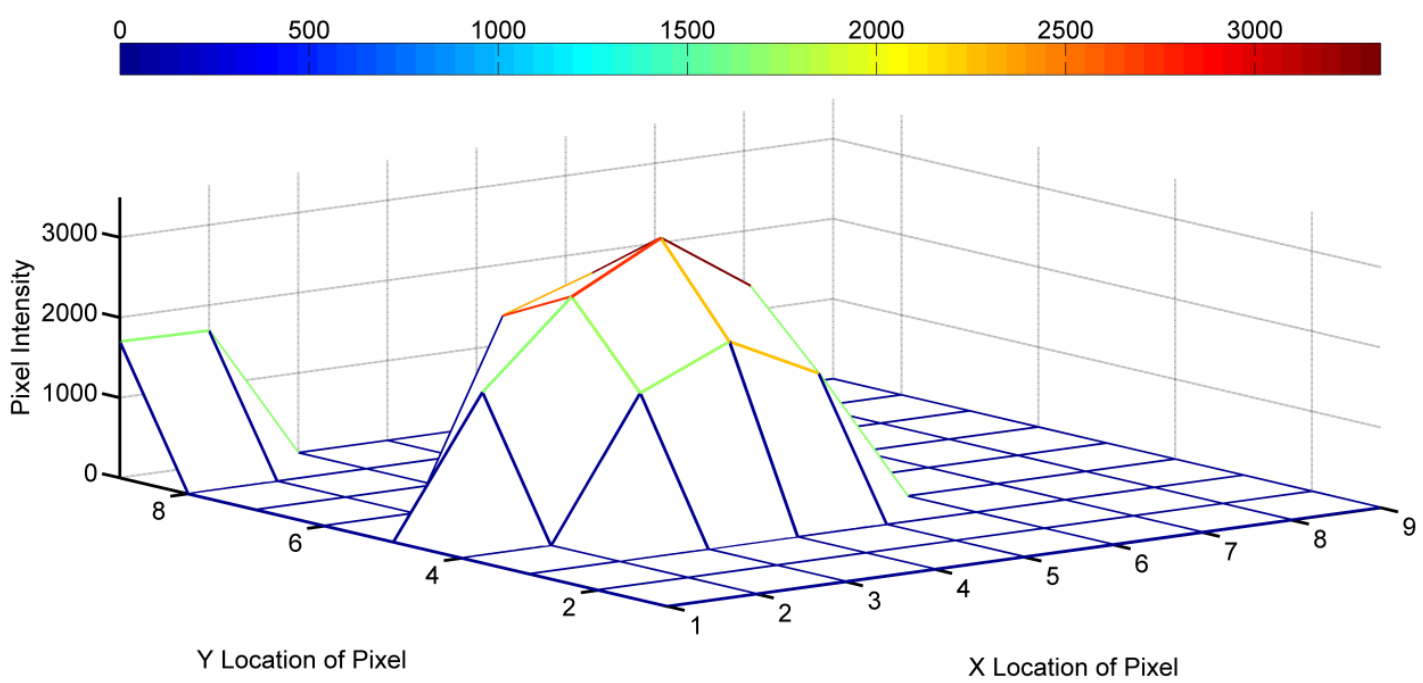

Figure 4.7 - 3D plot representing the pixel intensities of a fiducial marker in a $9 \times 9 \times 9$ pixel volume in the transverse plane. Fiducial marker pixels are more prominent with background activity set to 0 .

\subsubsection{Marker localization}

Three-dimensional localization $\left(\mathrm{x}_{\mathrm{g}}, \mathrm{y}_{\mathrm{g}}, \mathrm{z}_{\mathrm{g}}\right)$ of the marker was achieved using a weighted center of mass (COM) calculation on the thresholded image as demonstrated in equation: 4-2, 4-3, and 4-4.

$m_{i}=$ Intensity of individual pixel 


$$
\begin{aligned}
& \left(x_{i}, y_{i}, z_{i}\right)=\text { Location of individual pixel } \\
& \left(x_{g}, y_{g}, z_{g}\right)=\text { Location of center of mass } \\
& M=\sum_{i=1}^{n} m_{i}^{p} \\
& x_{g}=\frac{1}{M} \sum_{i=1}^{n} m_{i}^{p} x_{i} \\
& y_{g}=\frac{1}{M} \sum_{i=1}^{n} m_{i}^{p} y_{i} \\
& z_{g}=\frac{1}{M} \sum_{i=1}^{n} m_{i}^{p} z_{i}
\end{aligned}
$$

By increasing the power $p$ more emphasis was placed on the location of high activity pixels compared to the less active pixels in the neighbourhood. Integer values of $n$ ranging from 1 to 10 were initially used. Regardless, in cases with activity spread over more than one pixel an average location was resolved. COM calculations were performed on each time frame image independently to resolve the average position of the marker in 3D. 


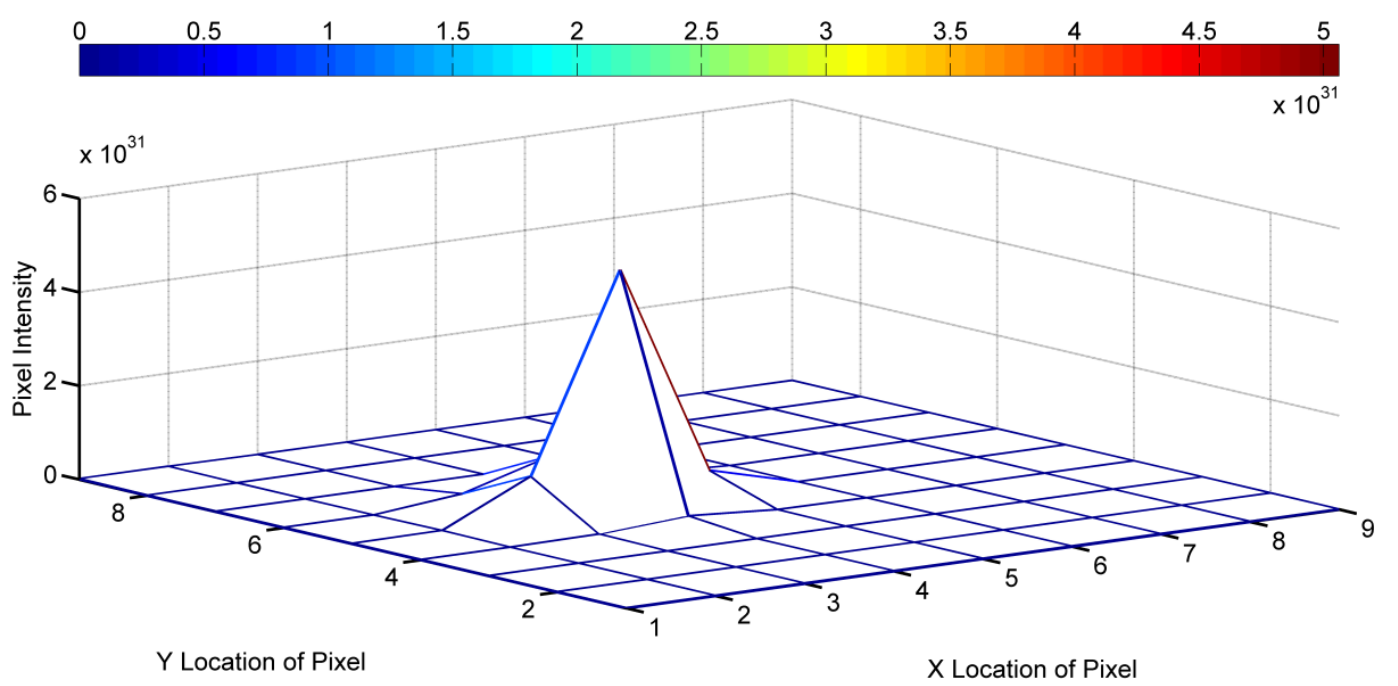

Figure 4.8 - 3D plot representing the pixel intensities of a fiducial marker in a 9x9 pixel volume in the transverse plane. Fiducial marker pixels have larger pixel intensities after being amplified by a power of $n=9$.

\subsubsection{Inter-frame Motion Detection}

Inter-frame motion was determined as the difference in COM between successive time frames. The motion distance (D) was calculated as the root-mean-squares of the motion in each Cartesian axis (X, Y, Z), demonstrated in Figure 4.9. Differences above a threshold amount (in $\mathrm{mm}$ ) were determined to be significant and could be used to flag a case with patient motion. This distance threshold is represented by $D$. Figure 4.10 shows an example of an inter-frame report. The change in threshold (D) frame-by-frame can be seen from time frame 1 to time frame 15. 
Time frame: 1
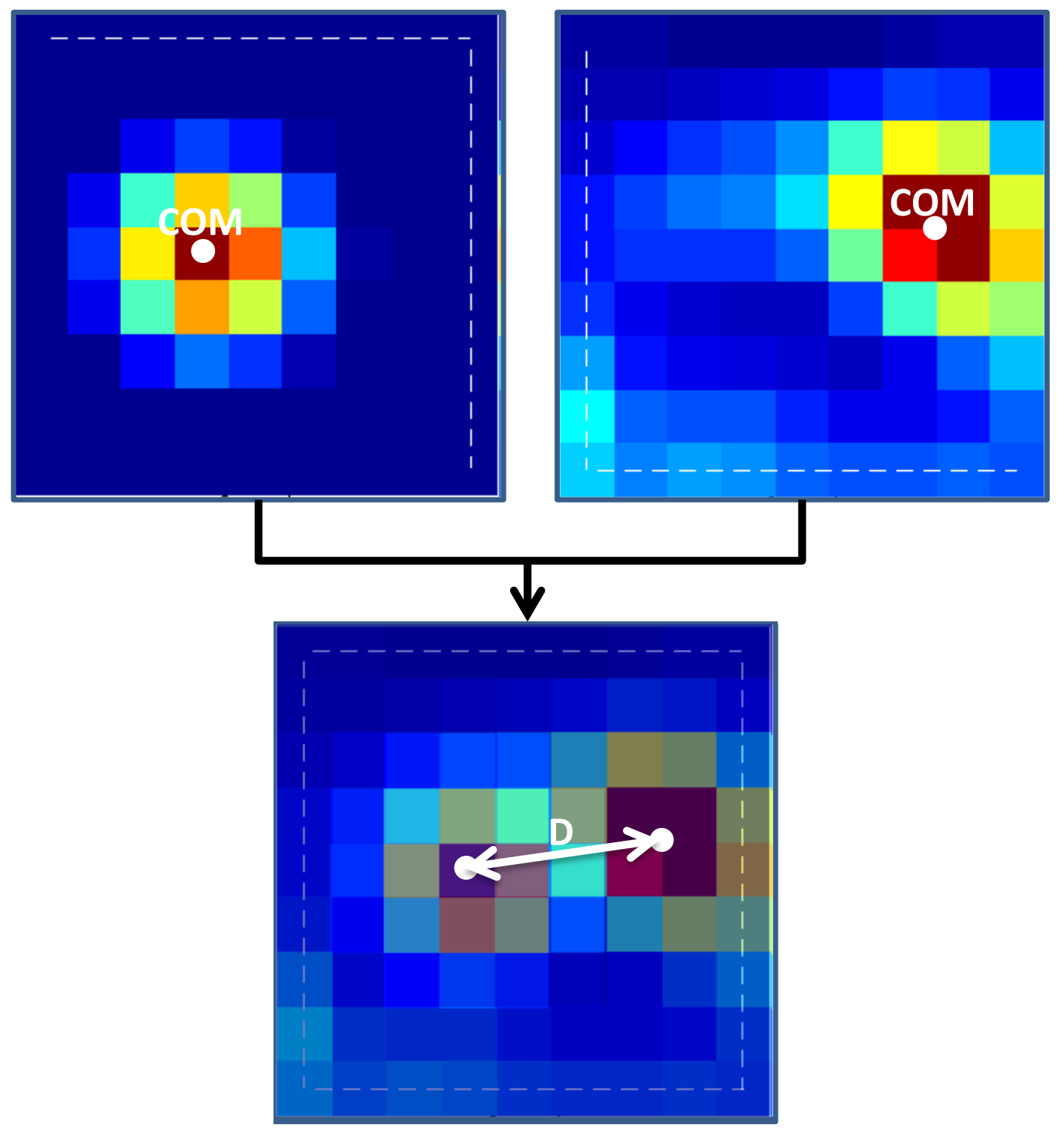

Min
Time frame: 2 


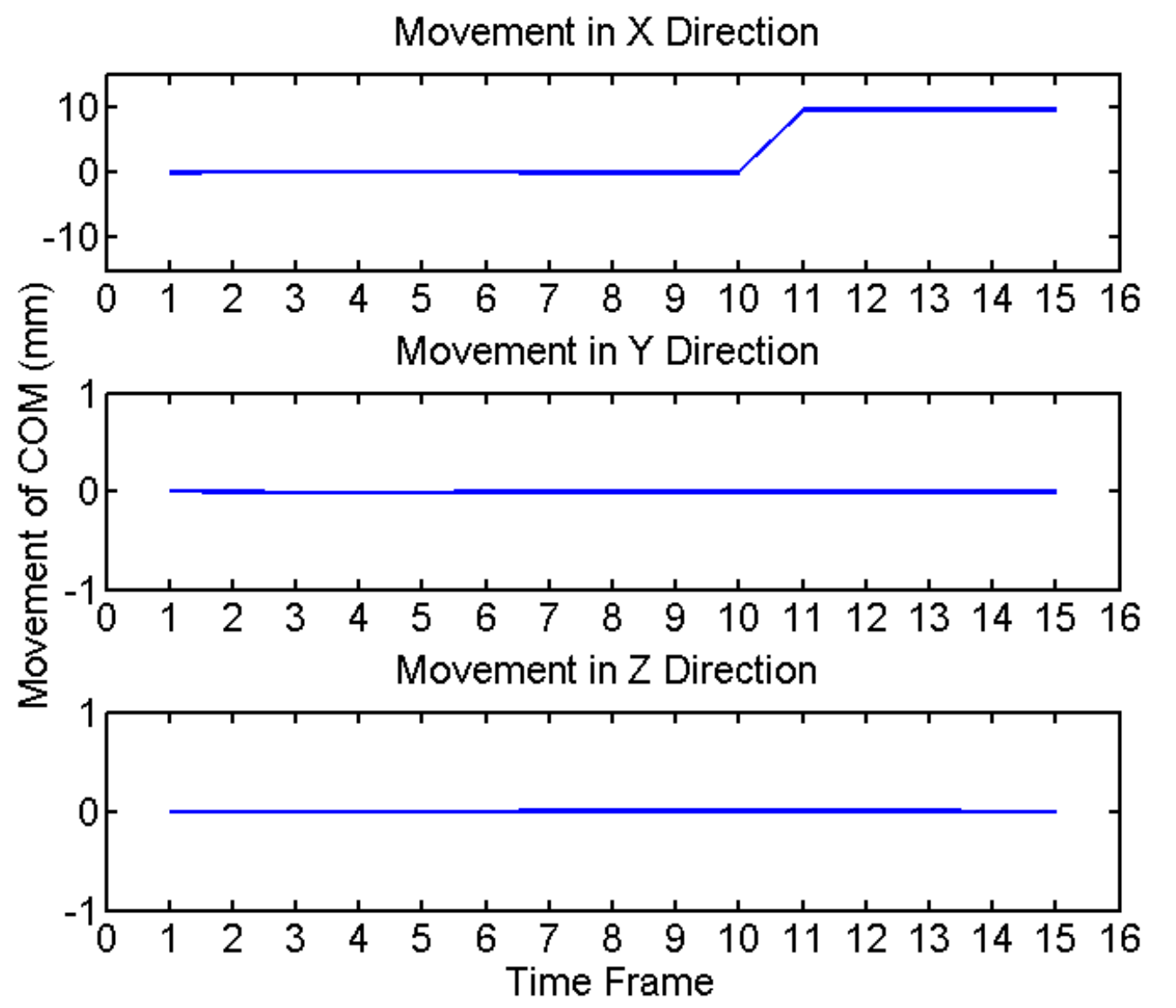

Figure 4.10 - Example of inter-frame motion report. A $10 \mathrm{~mm}$ change in COM is indicated between frame 10 and frame 11. The display notation is such that the first time frame is the reference (motion free).

In order to determine the threshold motion that could reliably indicate the presence of motion, receiver operator characterization (ROC) analysis was performed with the visually assessed motion serving as a reference truth. From the ROC analysis, sensitivity and specificity statistics were derived. 


\subsubsection{Intra-frame Algorithm}

The intra-frame algorithm estimated the degree of motion within a single frame by measuring the extent of the blurring of the fiducial marker. The COM was found in each frame as described in section 4.3.4.2 and a profile of activity fall-off with distance was created by plotting the peak pixel intensities $(\mathrm{I})$ as a function of radial distances $(\mathrm{x})$ from the COM. Due to the pixel property of the fiducial marker the COM is generally located near the pixel with the highest intensity and decreases with radial distance. The pixel intensities were normalized so the maximum intensity pixel was set to 1 . A curve (Equation 4-5 and demonstrated in Figure 4.11) was then fit to the image sampled profile.

$$
\begin{aligned}
& \mathrm{I}(\mathrm{x})=\left\{\begin{array}{cc}
1 & x<x_{0} \\
(1-c) \times e^{\left(-B\left(x-x_{0}\right)^{2}\right)}+c & x \geq x_{0}
\end{array}\right. \\
& B=\frac{\ln 2}{x_{1 / 2}^{2}} \rightarrow x_{1 / 2}=\sqrt{\frac{\ln 2}{B}}
\end{aligned}
$$




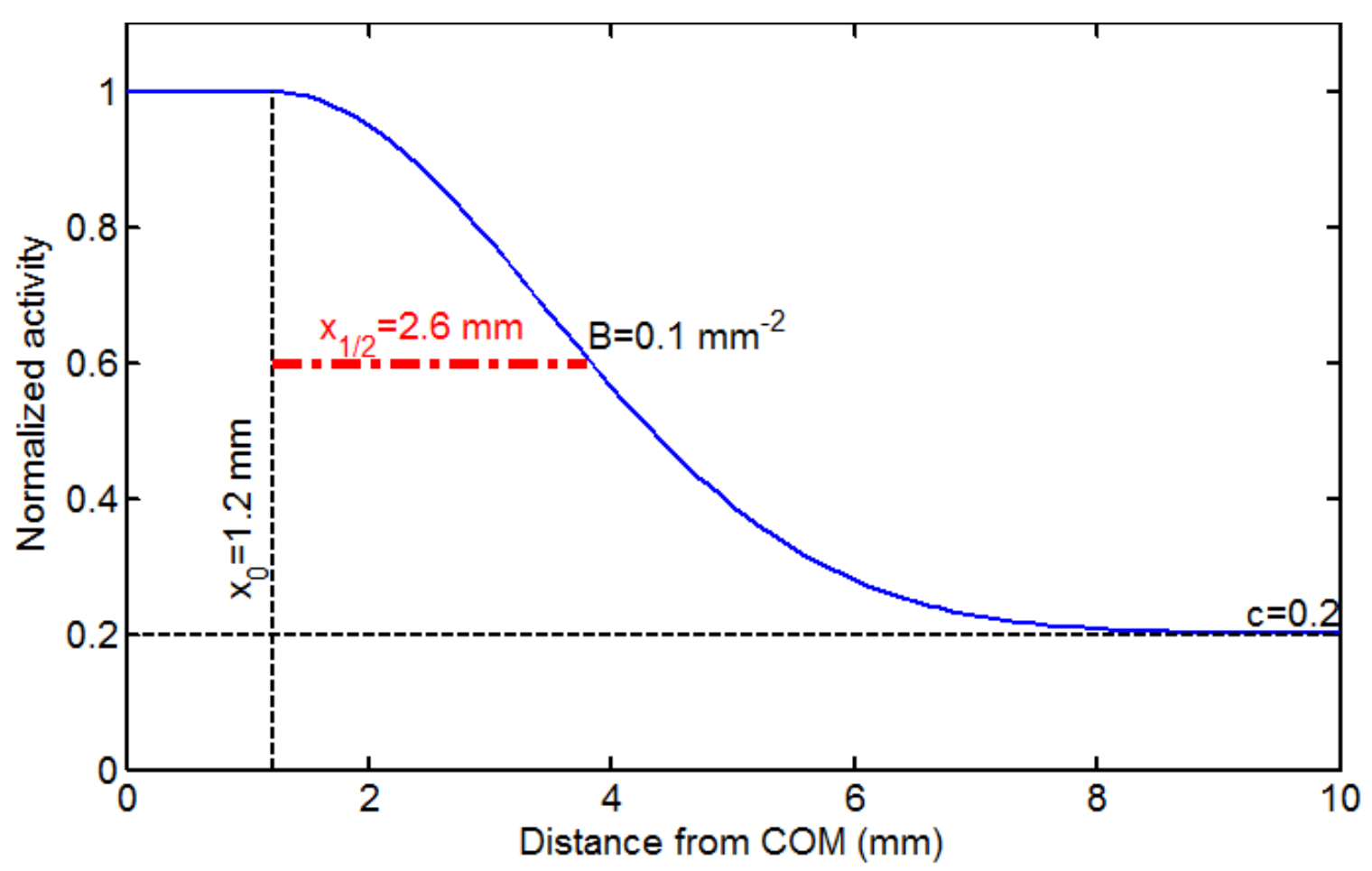

Figure 4.11 - A function fitted to activity fall-off profile to estimate marker blur resulting from motion

Parameter $\mathrm{x}_{0}$ is a distance $(\mathrm{mm})$ parameter of the breadth of the marker prior to activity fall-off and accounts both for the physical size of the marker and pixel size. Parameter c accounted for background activity.

The $B$ variable is a parameter of rate $\left(\mathrm{mm}^{-2}\right)$ of activity fall off with distance (breadth of profile) which was thresholded to determine the presence of motion blur. The exponential represents activity full-off with distance which is an approximation of PET image point-spread function. However the breadth is also dependent on by the amount of marker motion. Parameter $B$ can also be converted to a more intuitive form of $x_{1 / 2}$ 
(Equation 4-6), which is the distance in $\mathrm{mm}$ from where the profile is mid-intensity between peak and background, $\mathrm{c}$.

An image with fiducial marker blurring produces a larger $x_{1 / 2}$ value and represents a larger profile breadth as demonstrated in Figure 4.12 for a case without and with motion blur. ROC analysis with visual impression serving as reference truth was used to determine an $\mathrm{x}_{1 / 2}$ parameter cut-off for motion detection.
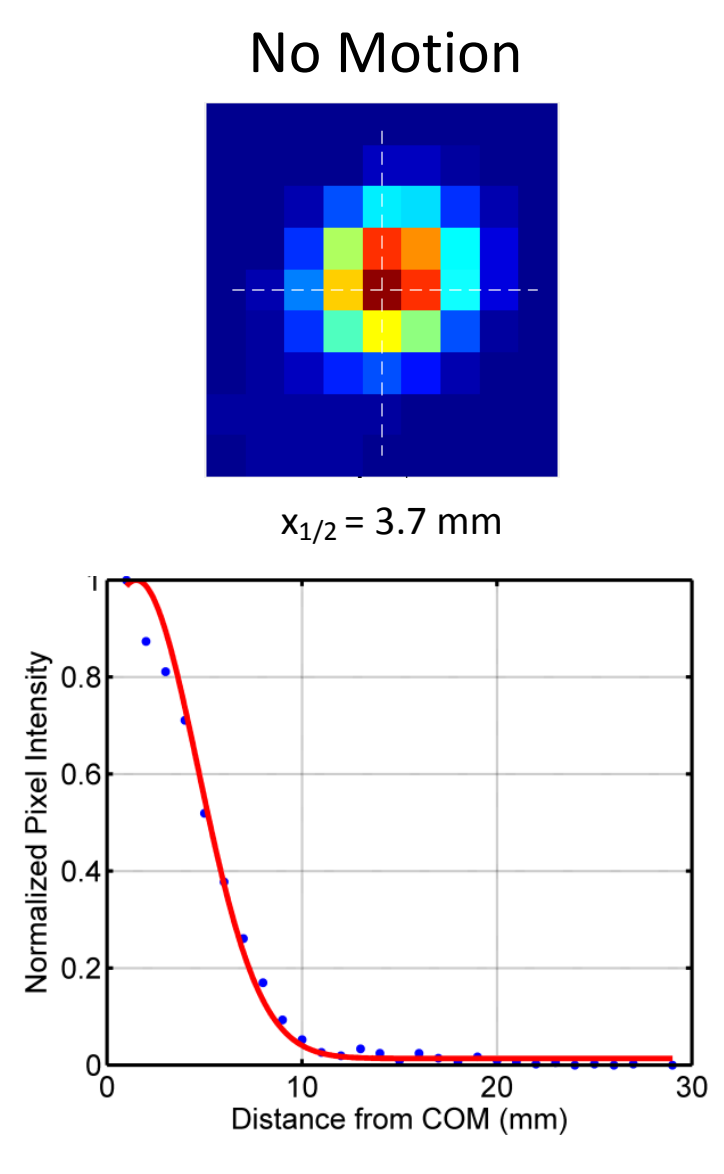

\section{Motion}

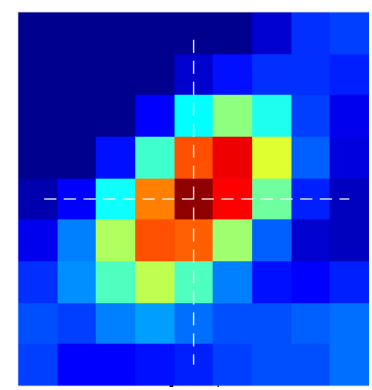

$\mathrm{x}_{1 / 2}=5.4 \mathrm{~mm}$

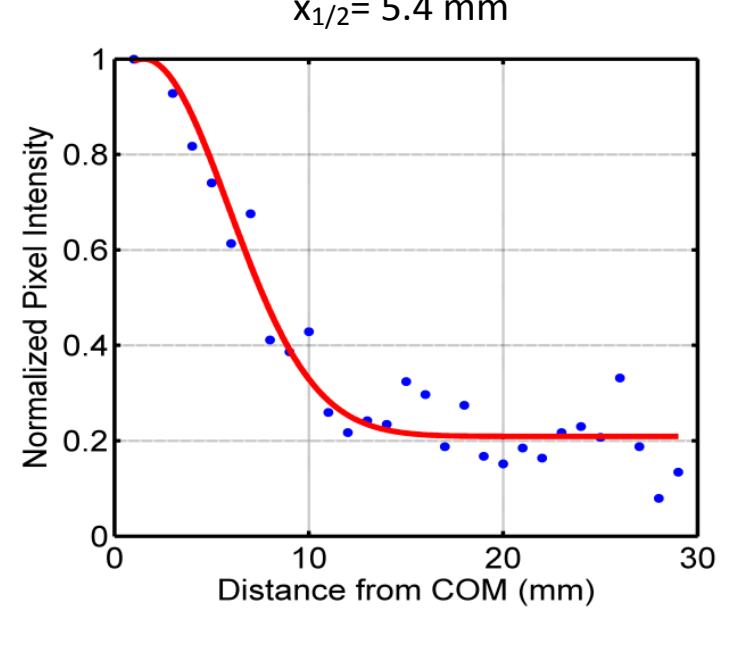

Figure 4.12 - Example of Intra-frame motion. The left hand image shows no motion with a lower $x_{1 / 2}$ threshold and profile. The right hand image shows an example of intra-frame motion with a higher $x_{1 / 2}$ threshold and its profile 


\subsubsection{Statistical Analysis}

To select optimal threshold values, ROC analysis was performed and the point on the curve with the shortest Euclidean distance to sensitivity $=100 \%$ and specificity $=100 \%$ was selected. Resulting sensitivities and specificities were then reported using this threshold along with $95 \%$ confidence intervals. The $95 \%$ confidence intervals were calculated using a bootstrap method where half of the image samples were randomly selected ten thousand times and then a ROC analysis was performed on each subset. The $95 \%$ confidence interval is reported as [lower bound, upper bound].

\subsubsection{COM Weighting Optimization}

As previously stated for COM calculation, by increasing the power $p$ more emphasis was placed on the location of the maximum activity pixel compared to averaging the location with less active, neighbouring pixels (Figure 4.8). We determined the optimal value for $p$ by performing ROC analysis with each integer value of $p=1$ to 10 on the entire dataset. A power $p$ which resulted in both high and stable (minimal variability with power change) accuracy, sensitivity and specificity values for both inter- and intra-frame algorithms was selected for this work.

\subsection{Results}

\subsubsection{Patient Data}

This study consisted of 130 images from 65 patients. All patients had at least one frame with intra-frame patient motion and at least one event with inter-frame motion at 
varying magnitudes. In total there were 367 events of inter-frame patient motion out of 1820 possible frame pairs detected visually and an additional 606 frames with intraframe motion blur out of 1950 frames.

\subsubsection{COM Weighting Optimization}

Inter and intra-frame sensitivity, specificity, and accuracy values are summarized in Figure 4.13 and Figure 4.14 respectively for varying $p$ power values. Figure 4.13 shows the increase in accuracy, sensitivity and specificity as the power $p$ increases when using the inter-frame algorithm on the full dataset. Figure 4.14 shows the increase in accuracy and sensitivity as the power $p$ increases when using the intra-frame algorithm on the full dataset. Based on these results we selected $p=9$, which provided a stable sensitivity, specificity and accuracy, as the optimal value to which all subsequent results pertain.

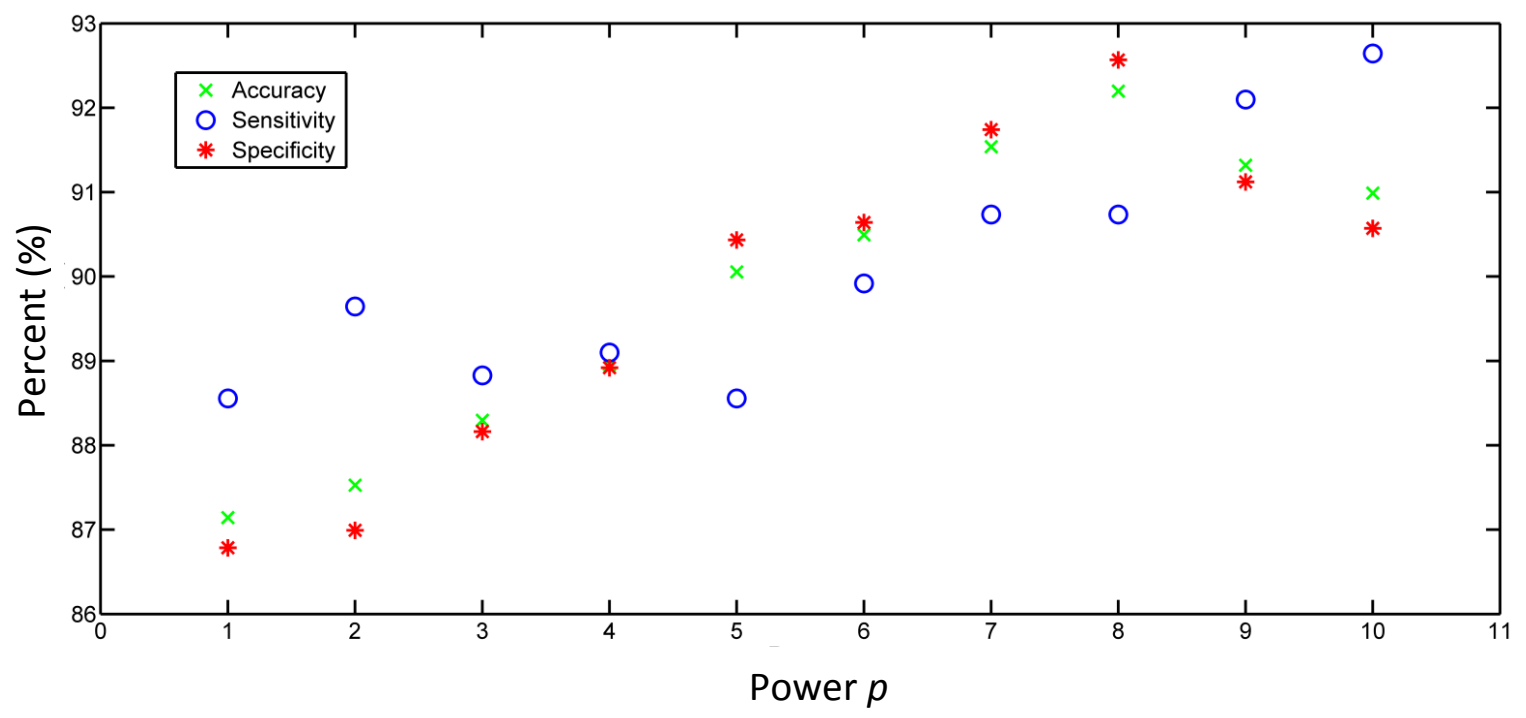

Figure 4.13 - Inter-frame motion detection accuracy, sensitivity and specificity for varying $p$ power values. 


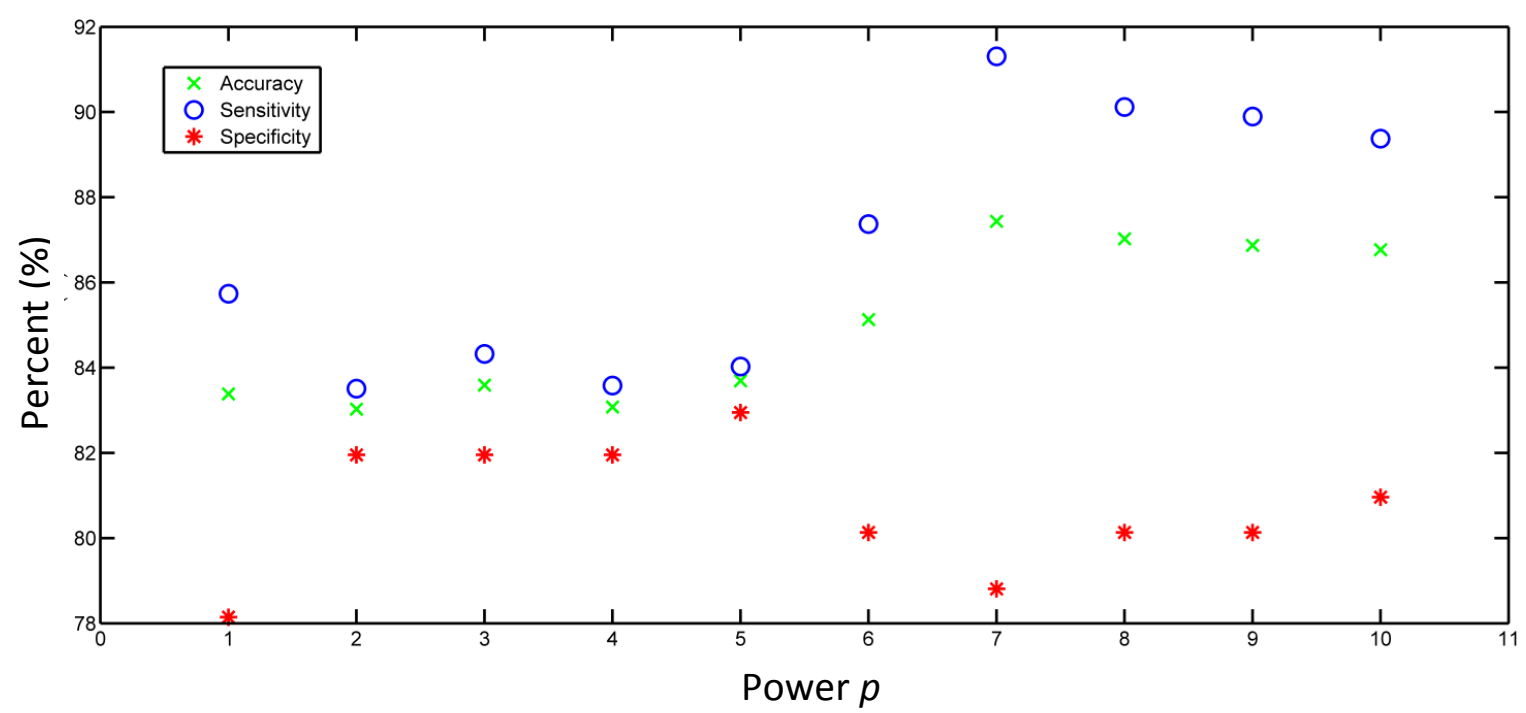

Figure 4.14 - Intra-frame motion detection accuracy, sensitivity and specificity for varying $p$ power values.

\subsubsection{Inter-frame Motion}

\subsubsection{Simulation data - motion magnitude precision}

Our simulations consisted of 716 neighbouring frame pairs without motion $(0 \mathrm{~mm})$ and 54 with motion $(9.3750 \mathrm{~mm})$. For the cases without motion, the maximum algorithm reported motion was $0.0139 \mathrm{~mm}$ whereas in the cases with motion the range of algorithm reported motion was between 9.3747 and $9.5399 \mathrm{~mm}$. Box-plots of the reported motion errors are shown in Figure 4.15. The maximum motion error was less than $5 \%$ of the pixel size $(3.125 \mathrm{~mm})$. No overlap in algorithm reported motions existed between the two cohorts, indicating ability for perfect classification of presence of motion in these simulation data. 


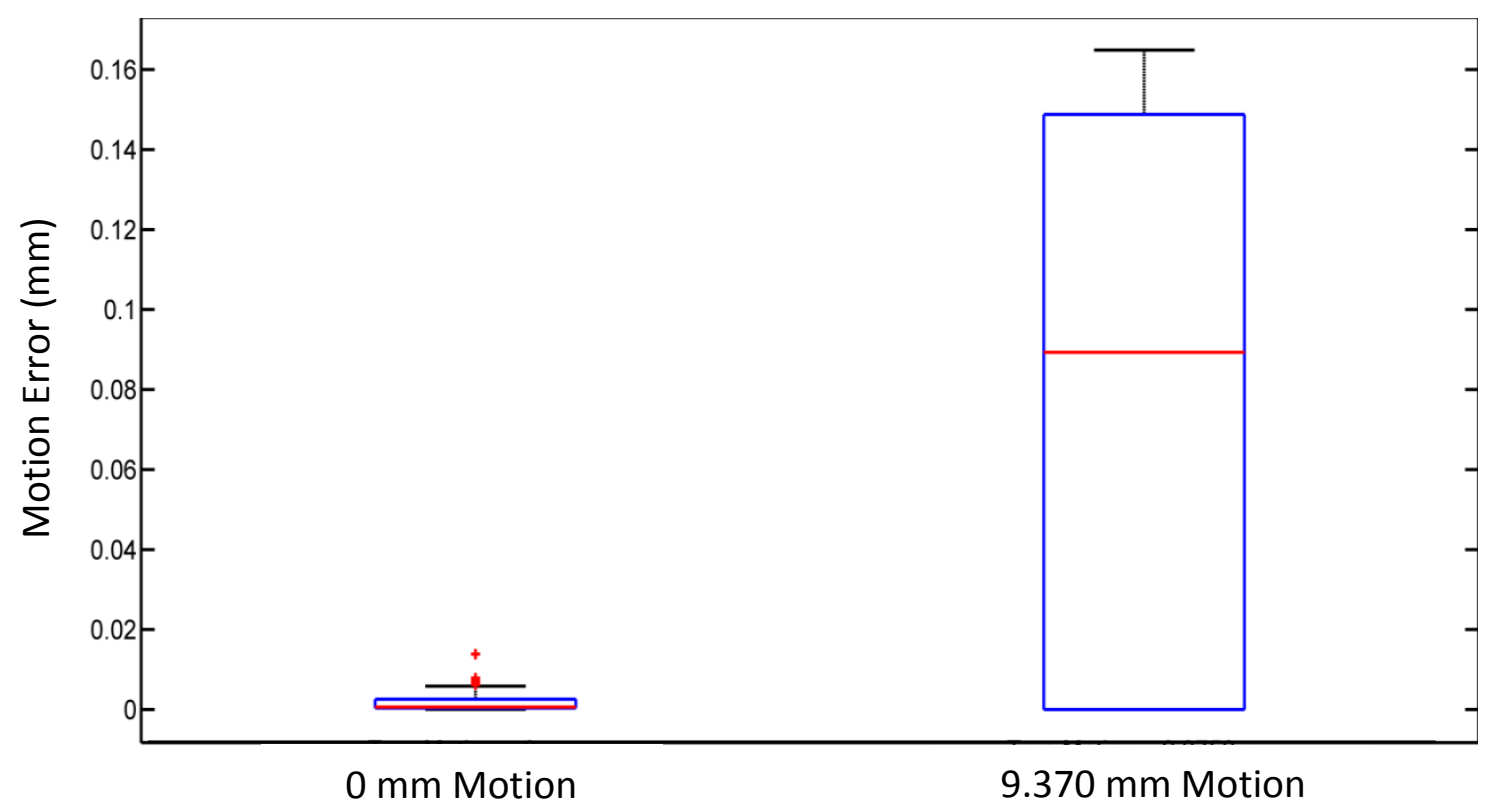

Figure 4.15 - Box plot showing error in algorithm reported inter-frame motion in simulated images with 0 and $9.3750 \mathrm{~mm}$ simulated motion ( $\mathrm{n}=716$ and 54 respectively). The maximum motion error was less than $5 \%$ pixel size $(3.125 \mathrm{~mm})$.

\subsubsection{Patient data}

Figure 4.16 shows the ROC curve for inter-frame motion COM distance threshold and the optimal threshold (blue circled at $D=2.7 \mathrm{~mm}$ ) resulting in a sensitivity of $91 \%$ [89\%, $94 \%]$ and a specificity of $91 \%$ [89\%, 94\%]. The area under the curve was determined to be 0.97 with an accuracy of $91 \%$. Algorithm versus visual detection rates are summarized in Table 4.1 using this $D$ threshold value. 


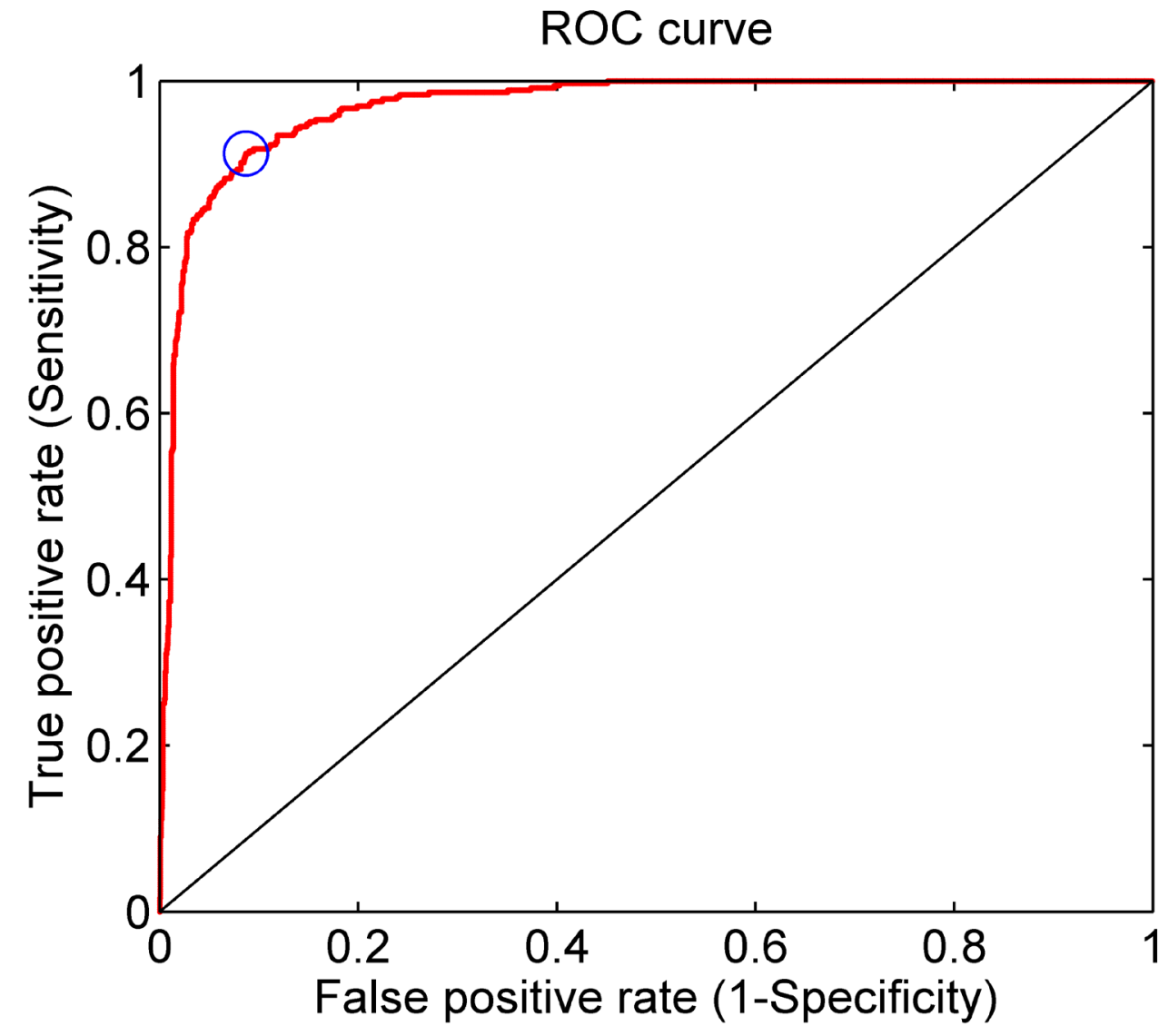

Figure 4.16 - ROC analysis for inter-frame algorithm. The blue circle represents an optimal threshold at $\mathrm{D}=\mathbf{2 . 7} \mathrm{mm}$.

Table 4.1 - Performance of inter-frame motion detection algorithm ( $n=1820$ frame pairs from 130 images)

\begin{tabular}{|c|c|c|}
\hline Algorithm & $\begin{array}{c}\text { Visual } \\
\text { Motion }\end{array}$ & $\begin{array}{c}\text { Visual } \\
\text { No Motion }\end{array}$ \\
\hline $\begin{array}{c}\text { Motion } \\
\text { Noorithm }\end{array}$ & 335 & 126 \\
\hline
\end{tabular}




\subsubsection{Intra-frame Motion}

Figure 4.17 shows a ROC curve for intra-frame motion with the optimal motion detection threshold (blue circled at $x_{1 / 2}=4.4 \mathrm{~mm}$ ) resulting in a sensitivity of $82 \%$ [ $80 \%$, $86 \%]$ and a specificity of $90 \%$ [86\%, 92\%]. The area under the curve was determined to be 0.90 with an accuracy of $87 \%$. Algorithm versus visual detection rates are summarized in Table 4.2 using this B threshold value.

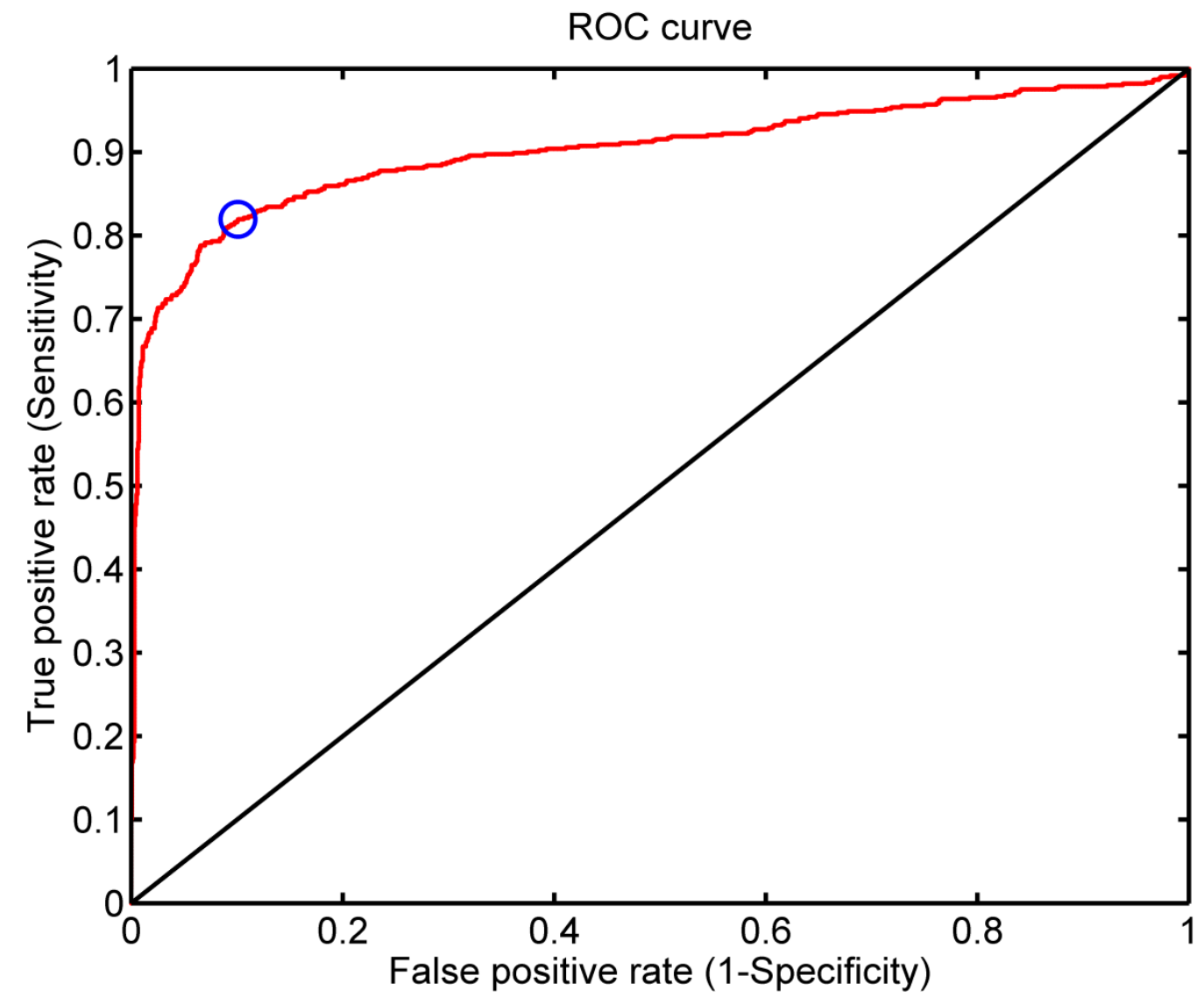

Figure 4.17 - ROC analysis for intra-frame algorithm. The blue circle represents a threshold at $x_{1 / 2}=4.4 \mathrm{~mm}$. 
Table 4.2 - Performance of intra-frame motion detection algorithm ( $n=1950$ frames in 130 images)

\begin{tabular}{|c|c|c|}
\hline & $\begin{array}{c}\text { Visual } \\
\text { Motion }\end{array}$ & $\begin{array}{c}\text { Visual } \\
\text { No Motion }\end{array}$ \\
\hline $\begin{array}{c}\text { Algorithm } \\
\text { Motion }\end{array}$ & 497 & 137 \\
\hline $\begin{array}{c}\text { Algorithm } \\
\text { No Motion }\end{array}$ & 108 & 1208 \\
\hline
\end{tabular}

The boxplots, in Figure 4.18 , show the variance in $\mathrm{x}_{1 / 2}$ values for frames with and without intra-frame motion. The $\mathrm{x}_{1 / 2}=4.4 \mathrm{~mm}$ threshold obtained from the ROC analysis separates the two sets of data.

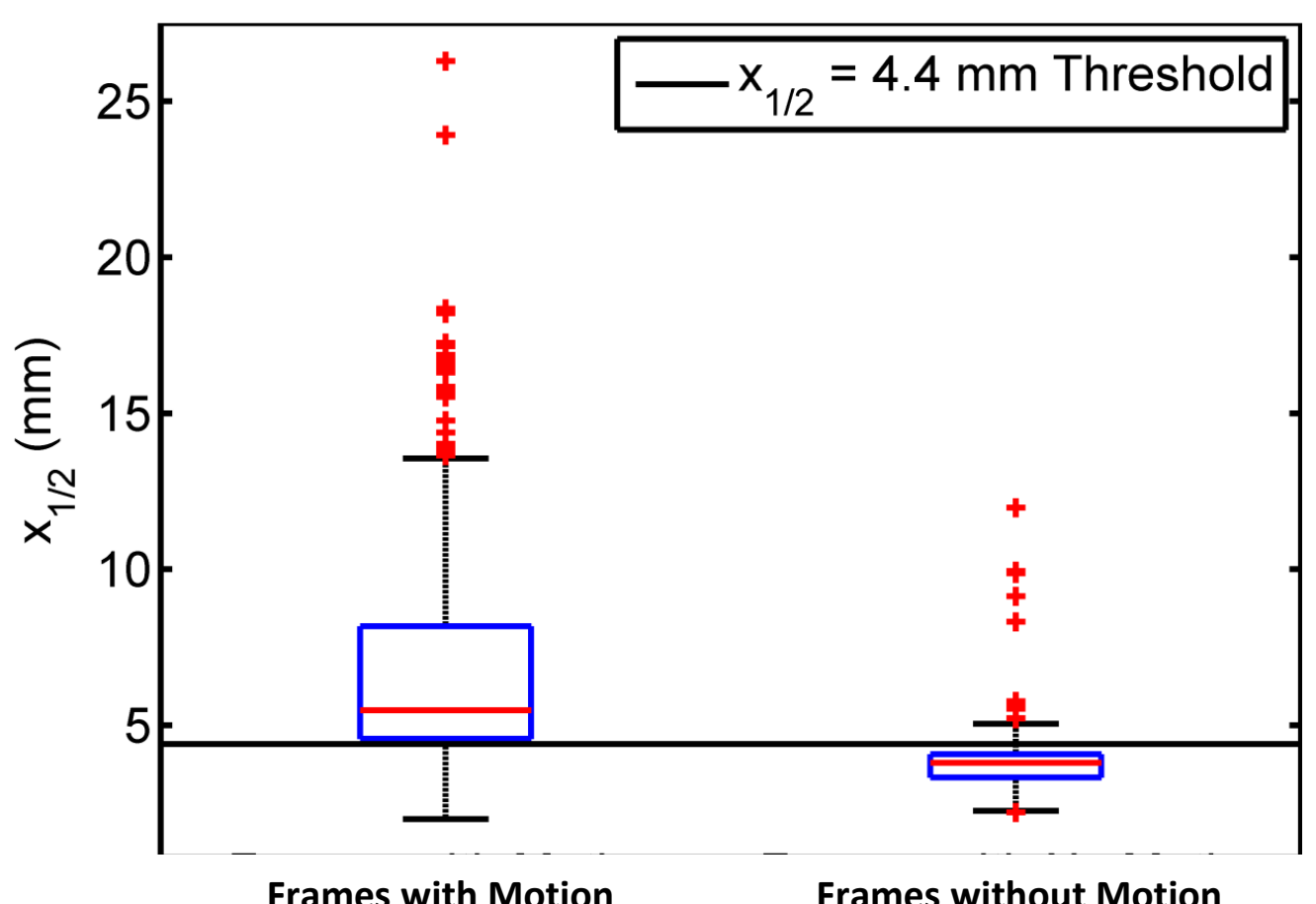

Figure 4.18 - Boxplot describing $x_{1 / 2}$ values which represent motion and no motion cases. $x_{1 / 2}=4.4 \mathrm{~mm}$ threshold is represented by the black line separating the two motion cases 


\subsection{Discussion}

This work describes and evaluates two automatic algorithms to detect fiducial marker motion in dynamic cardiac PET images. The inter-frame algorithm assessed marker motion between neighbouring time frames based on changes in marker 3D localization using COM approximation. The intra-frame algorithm assessed motion within an image frame by assessing the blurriness of the marker image. Optimal motion detection thresholds were empirically determined. The resulting accuracy was found to be $91 \%$ and $87 \%$ respectively using visual assessment as a reference truth. These accuracy rates are sufficiently high to be of benefit for quality control purposes in a high throughput clinical setting in which visualization of each dynamic sequence is not practical.

In our study, we determined that inter-frame motion was detected with an accuracy of 91\% using a threshold of $2.7 \mathrm{~mm}$ COM motion between successive frames. The size of each voxel was $3.125 \mathrm{~mm}$ and the transverse plane and $3.200 \mathrm{~mm}$ axially and image spatial resolution was $\sim 10 \mathrm{~mm}$. Therefore the motion detection threshold was smaller than one voxel in size and well below the image spatial resolution. Since $<2$ voxel motion is unlikely to have meaningful clinical impact, it is likely that increasing the threshold value would result in a lower false-positive rate (improved specificity) without dramatically reducing sensitivity. Likewise the optimal $x_{1 / 2}$ parameter threshold value for intra-frame motion detection was determined to be $4.4 \mathrm{~mm}$, well below the image spatial resolution, with an accuracy of $87 \%$. Once again, using a higher $x_{1 / 2}$ value 
threshold could improve the algorithm specificity to detect motion without clinically significant detriment to sensitivity.

Assuming that motion less than 2 pixels in size are inconsequential the resulting interframe specificity increased from $91 \%$ to $99 \%$ (or $94 \%$ for motion less than 1 pixel). Assuming that motion less than the image spatial resolution $(10 \mathrm{~mm})$ are inconsequential the resulting intra-frame motion specificity increase from $90 \%$ to $99 \%$ respectively. Nevertheless, further validation is required to establish optimal threshold values, preferably with a reference truth such as external tracking that is able to quantify the magnitude of marker motion.

\subsubsection{Clinical Feasibility}

The advantage of this study over previous work is the use of a low-cost low-dose marker which limits radiation exposure to the patient and instrumentation complexity. The algorithms are implemented post-reconstruction therefore are vendor independent and can be used to track fiducial markers in a larger range of applications (e.g. brain, oncology, and organ motion) and imaging modalities are not limited to PET.

The execution times for these algorithms were 0.9 seconds per dynamic image sequence on a modern laptop, including overheads such as loading image data into memory. Therefore, efficient integration of these algorithms into MBF quantification software would result in negligible incremental processing time. Furthermore, affixing the fiducial marker to the patient takes only seconds and does not interfere with the 
imaging process. Thus we conclude that these algorithms may be applicable for routine quality control in MBF studies.

The algorithm can be included as a separate utility for batch processing studies and generating quality control indicators, or more practically integrated into software packages for processing dynamic image sequences. While physician confidence may increase in cases without indicated motion, cases with motion would need more thorough interpretation in the context of possible motion or disregarding of dynamic analysis results.

\subsubsection{Other applications}

The algorithm could easily be expanded to track multiple markers by introducing multiple VOI around each marker. Multiple markers have been used to track head motion and respiratory motion to fully characterize the direction and magnitude of the motion to guide correction. Future work would consist of tracking multiple markers on the torso to determine if heart motion can be fully characterize during translational and rotational motion.

The intra-frame motion detection algorithm could be applied to static (PET or SPECT) MPI quality control without any substantial modifications. As with dynamic imaging static imaging for MPI interpretation is performed over long time intervals (e.g. $6 \mathrm{~min}$ ) in which patient motion can manifest itself as a directional blur which can produce uptake 
deficits consistent with CAD [62]. Thus this algorithm can have important QC implications on the most commonly used procedure in nuclear medicine.

\subsubsection{Limitations and Future Work}

As previously stated, visual interpretation is vulnerable to viewer bias, errors, and is limited to qualitative assessment. Nevertheless, it is not possible to induce motion in clinical studies and another (external) reference was not available to us for this study.

A current limitation with this study is that motion larger than the VOI $9 \times 9 \times 9$ pixel volume) was not explicitly supported. This would correspond to extremely large patient motion in excess of $3 \mathrm{~cm}$ which would likely result in obvious MBF and MPI artifacts. If blurring of the fiducial marker exceeds the VOI it would still be detected as motion but error in the detected magnitude will exist. Partial movement of the marker outside the VOI could result in slight error in COM calculation and thus the detected magnitude of motion. Nevertheless, this limitation could be addressed by updating the VOI position in each time frame depending on COM localization in the previous frame or by increasing the VOI size. We tried to reduce the effects of spill in body activity in the VOI but this is also a source of error when calculating the COM and thus detecting the magnitude of motion for both algorithms. The use of two separate algorithms to detect inter- and intra-frame motion may not be ideal. A potential simplification could be to superimpose all 15 time frame images and then attempt to detect motion using the intra-frame algorithm. However it is likely that sensitivity would be adversely impacted. For this 
reason we did not evaluate the method in this work, but may be worthy to pursue in subsequent work.

The authors recognize that the noise-free NCAT simulations used in this study are limited in their accuracy and that they did not simulate intra-frame motion. Future work is planned to expand the evaluation of these algorithms using more complete simulation models including monte-carlo simulation of emission, attenuation, scatter and detection, realistic noise models, iterative image reconstruction, and a broader range of motion models.

A final important limitation is that this study used the same data set to optimize detection thresholds $(p=9)$ and to characterize algorithm performance. Ideally, separate datasets would be used. However, due to the tedious work required to collect image data and visually assess the presence of motion in each and between time frames this was not performed. Follow-up characterization should be performed with a separate dataset. Nevertheless we believe that performance measures reported in this work are representative of true clinical scenarios.

\subsection{Conclusion}

We have developed and validated inter- and intra-frame motion detection algorithms to track fiducial markers in reconstructed dynamic PET images. These algorithms can be easily incorporated into analysis software as an effective added quality control measure without detriment to clinical workflow. 


\section{Image Decomposition Based Heart Motion Detection}

\subsection{Background}

\subsubsection{Matrix Decomposition - Dynamic Image Decomposition}

Dynamic images consist of 4 dimensions including 3 spatial dimensions and a time dimension. A pixel in a dynamic image therefore also has a time component and may be referred to as a dixel, representing activity in a defined volume over a sequence of time frames and can be represented as a vector. Image decomposition can unveil spatial patterns in a dynamic image based on the characteristic temporal patterns. These temporal patterns may be predefined (e.g. Fourier analysis, wavelet) or determined from the image data using models that provide a unique solution to the decomposition problem.

Image decomposition techniques that are based on the temporal pattern of each dixel usually begin by collapsing the volume into a $2 \mathrm{D}$ matrix. The columns of the dataset matrix $(\mathrm{Y})$ represent that time dimension while the rows represent the image pixels (i.e. spatial dimensions). The image matrix, $\mathrm{Y}$, is then decomposed into two matrices: $F$ represents the column wise temporal factor matrix, and $S$ is the corresponding structures matrix which represents the spatial distribution of each component. The residual signal and random noise which is not accounted for by the components is represented by matrix $\in$ which has the same dimensions as the original image. The number of components to resolve must be determined in advance and corresponds to 
the number of columns in $F$ and number of rows is $S$. The decomposition framework may be represented using the following equation (5-1):

$$
Y=F S+\epsilon
$$

Figure 5.1 illustrates how image decomposition works mathematically. In this example the original image is decomposed into three components consisting of both spatial patterns (structures) and corresponding temporal time-curves (factors). The remaining signal in the original image which was not represented by the decomposed components is the residue image. One potential advantage of image decomposition is that it accounts for all dixels in the image simultaneously. 

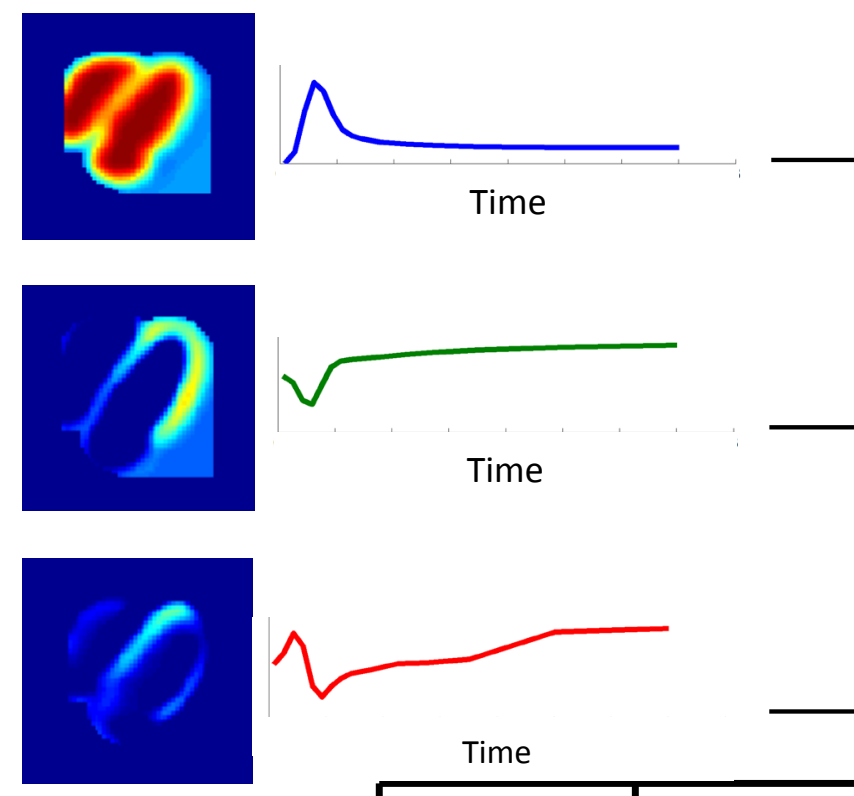

\section{Time}
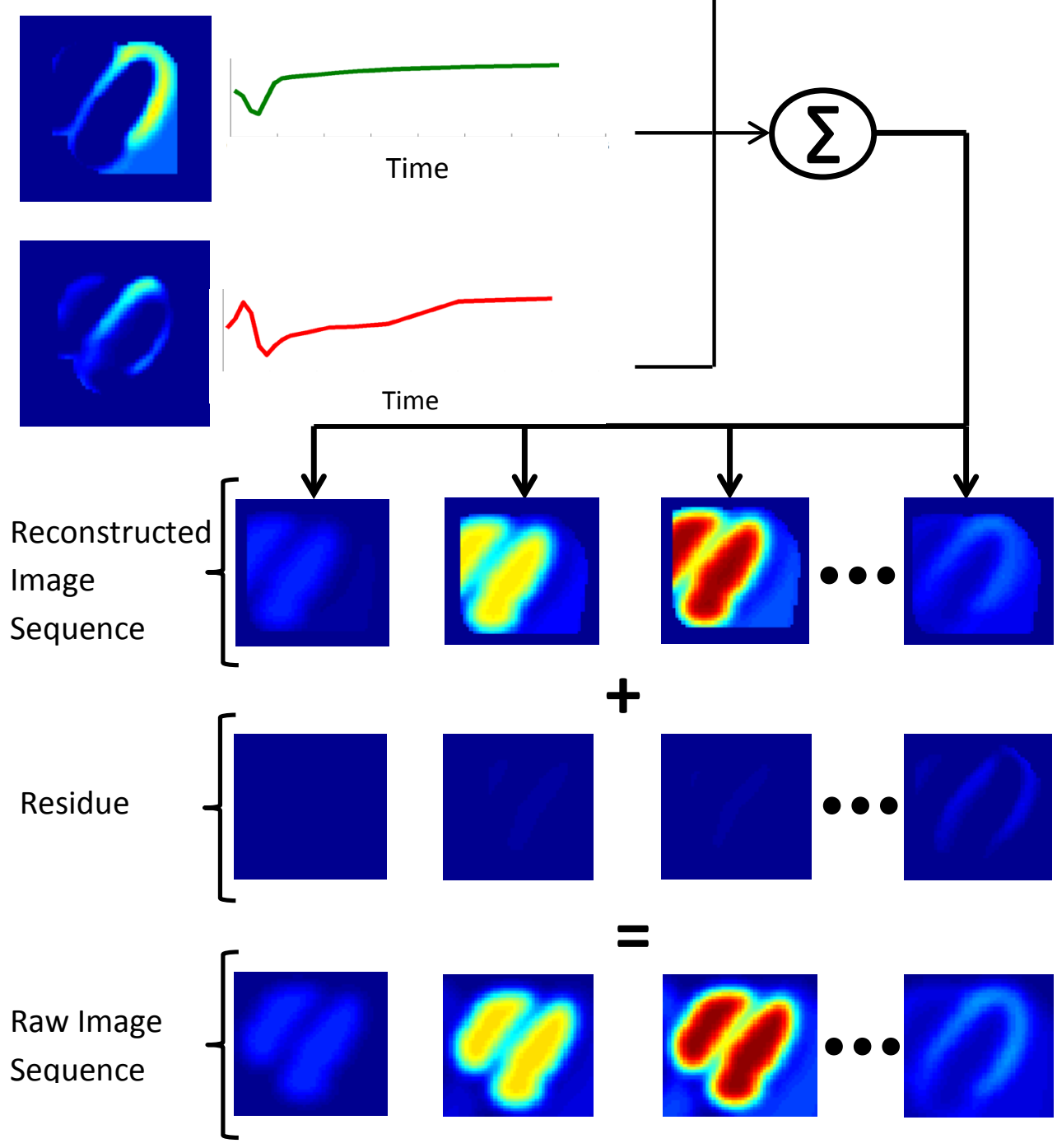

Time Frames

Figure 5.1 - Image decomposition diagram: Summed products of spatial structures and temporal factors (top left) produce an estimated reconstructed image sequence which combined with the residue image sequence represents the original raw dynamic image sequence (bottom). Image decomposition methods simultaneously determine 
the structures and factors from the raw image sequence. The residue was analyzed for patterns indicating undesired subject motion during image acquisition.

Image decomposition has been used on dynamic nuclear medical images in the past. For example, several studies used factor analysis of dynamic PET images to create factor images and TACs to estimate parameters for kinetic modelling [61-64]. Another study investigated two factor analysis of dynamic structures (FADS) methods for use of semiautomatic estimation of activity in cardiac dynamic SPECT images [67]. Image decomposition is not a unique method, with many different approaches available depending on the application and goal.

\subsubsection{Principle Component Analysis (PCA)}

Principle component analysis is a simple and commonly used decomposition method. It has a well-defined solution that creates eigenvectors of the covariance matrix of the data so as to account for as much signal (variance) in the original image matrix given the number of desired components [66-68]. PCA can easily and quickly be processed on a modern computer making it one of the most widely used methods for decomposition.

PCA has been used for data compression and the reduction of noise in images. The resulting decomposition quantifies the variance represented by the Eigenvectors basisfunctions (components) through the relative magnitudes of the corresponding Eigenvalues of the solution. 


\subsubsection{Number of Components}

The number of factors into which the image must be decomposed must be predetermined. The number of desired components is mainly dependant on the quality of the image (degree of noise), number of prominent structures (organs/blood/myocardium), structure kinetics (i.e. temporal patterns) and time frame intervals. If too few factors are resolved, then structures with similar kinetics may be lumped together while too many factors can lead to structures being split into multiple components. Furthermore, a large number of factors makes the decomposition process more susceptible to noise [71].

The number of factors can be selected based on the relative image sequence variance represented by each component. The relative variance is measured by the respective eigenvalues of the covariance matrix, determined using PCA. The eigenvalues can be normalized to unity sum and then cumulatively summed in decreasing order of magnitude to account for the total image variance accounted by $\mathrm{n}$ components. One means by which the number of components to include is resolved is using a userselected image variance value to include in the solution (or reject to the residue) (Figure $5.2)$. 


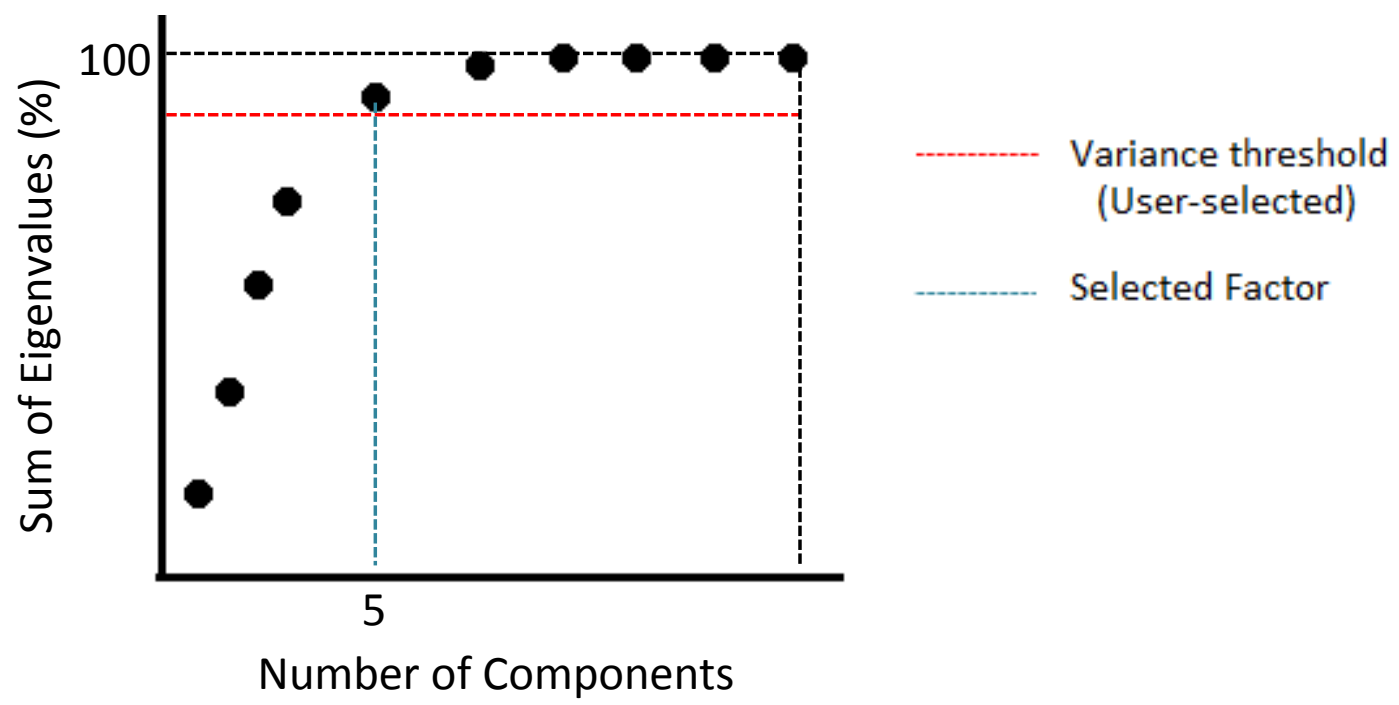

Figure 5.2: Method for selecting the number of components to resolve based on total cumulative variance (Eigenvalues) as determined by PCA. The user selects a desired total variance (range: $0-100 \%$ ) and the closest number of components to achieve this threshold is used.

Anecdotal data from previous work involving decomposition of dynamic PET [72] has suggested that image matrix decomposition may be able to expose the presence of motion as a pattern in the error image, however this hypothesis has not been explored in further detail. Figure 5.3 demonstrates a simulated dynamic PET image which has motion in its last time frame (240 $\mathrm{s}$ in the $+x$ direction). This dynamic sequence (left most column) has been decomposed using a PCA algorithm (2 factors). The most important components are decomposed in the first two components (factors and structures) which can be seen in Figure 5.3 by the two structure columns. It can be seen in the $2^{\text {nd }}$ factor that the myocardial wall is visible in that decomposition stage. It is also important to note that the structures in the dynamic image are not fully decomposed, 
as evident by the prominent structure (red structure in the last two time frames) in the residue. The structure resembles the myocardium and is consistent with heart motion in last time frame. In this chapter, we sought to evaluate whether dynamic image decomposition may be used to detect the presence of motion.

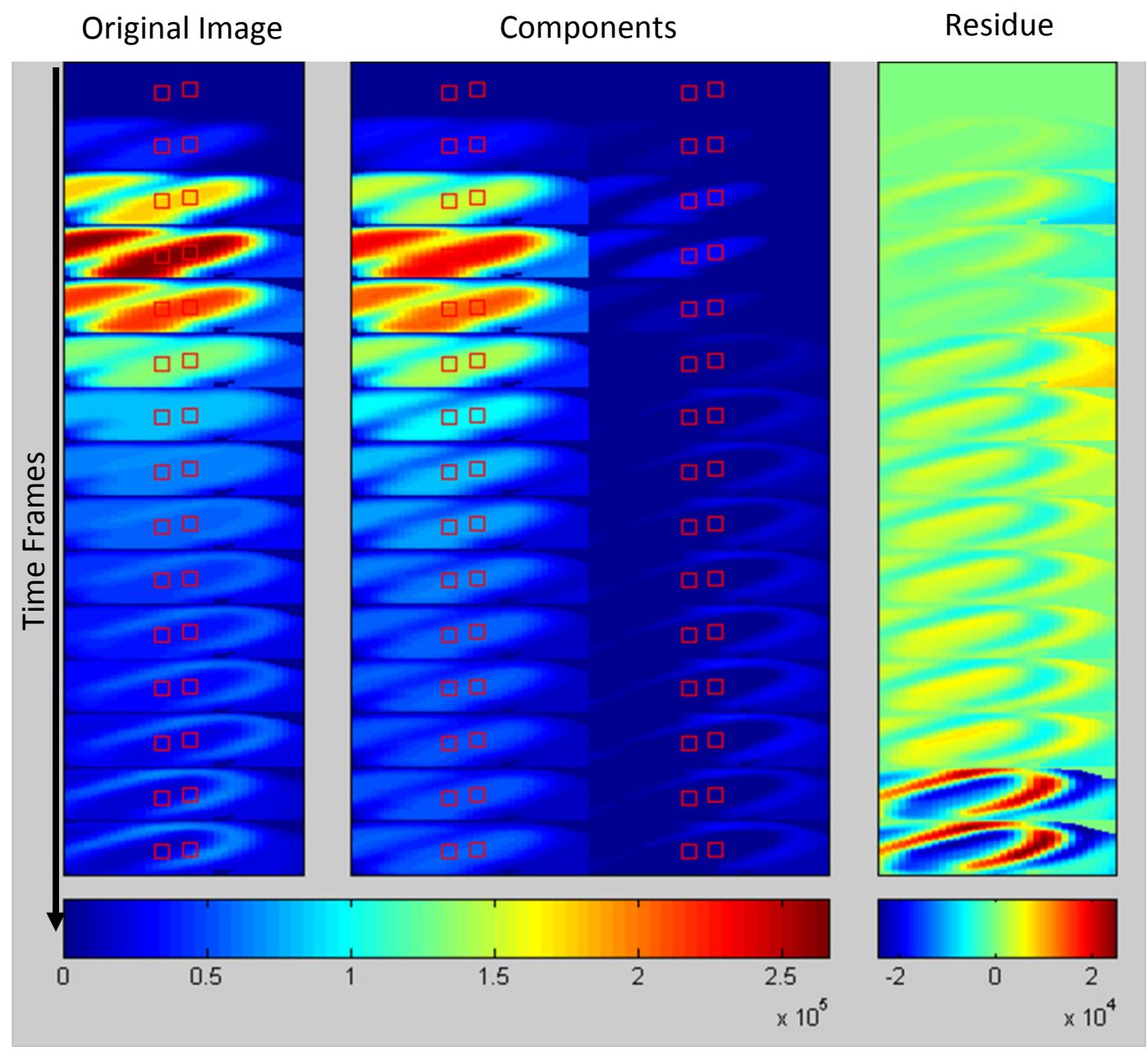

Figure 5.3 - An example of PCA image decomposition using 2 factors in a simulated dynamic image sequence with motion (left). The blood and myocardium wall patterns can be seen in the two components. A myocardium structure can also be seen at in the last two time frames of the residue image which corresponds to simulated motion. Note the separate color scale on the residue image. 


\subsection{Objective}

This chapter is an early investigation of whether principle component analysis, an image decomposition method, holds promise for detection of heart motion in dynamic ${ }^{82} \mathrm{Rb}$ PET based on patterns in the residue image.

\subsection{Methods}

To test whether heart motion could be detected using patterns in the residue of PCA decomposition of dynamic image data, a series of simulated and patient data were processed with PCA and the residue was analyzed. These datasets are described below, followed by a description of the analysis.

\subsubsection{Noise-free Simulation}

108 noise-free NURBS-based cardiac-torso (NCAT) $[56,57]$ numerical-phantom dynamic image sequences were generated. 54 images had $\pm 0.9375 \mathrm{~cm}$ simulated motion and 54 images had $\pm 1.8750 \mathrm{~cm}$ simulated motion (referred to as 1 and $2 \mathrm{~cm}$ respectively). The images were generated using the same methods as section 4.3.1. An example of interframe motion between two frames in a noise-free simulation can be seen in Figure 5.4. 
Time Frame: 13

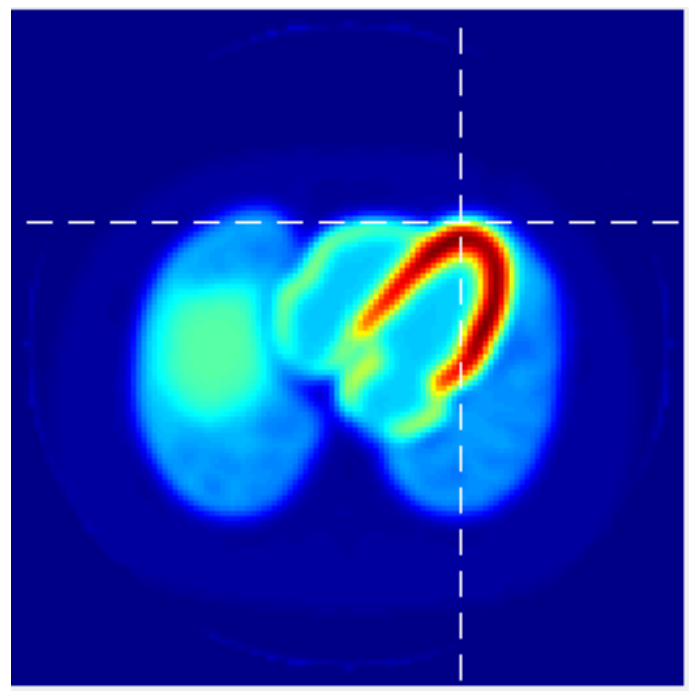

Time Frame: 14

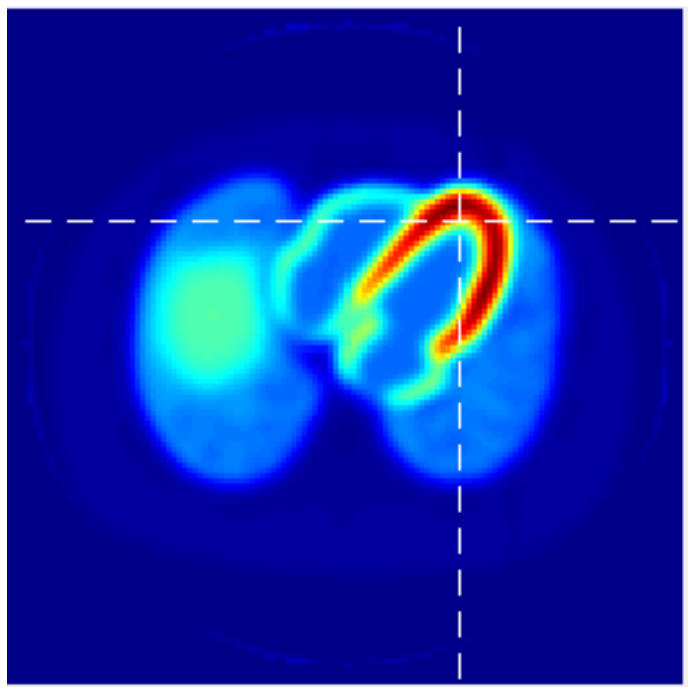

Max

Figure 5.4 - The transverse image shows an NCAT noise-free simulation. The image on the left (frame 13) is without motion and the image on the right (frame 14) is shifted by $2 \mathrm{~cm}$ in the +y direction (at $240 \mathrm{~s}$ ). The myocardium can be seen in red. The crosshairs are fixed between time frames.

\subsubsection{Noisy Simulation}

A second, but similar set of 108 NCAT $[56,57]$ based dynamic image sequences were generated with noise. As with the noise-free simulations, NCAT dynamic images were forward projected (128 projections) using the radon transfer function. Noise (modeled for the GE advance PET scanner and a $20 \mathrm{~cm}$ diameter phantom) was then added to the sinograms using the analytical simulator ASIM (University of Washington, Seattle, WA)

[73] and then reconstructed using iterative filtered back projection with Software for 
Tomographic Image Reconstruction (STIR) [74]. ASIM is a software package which can be used to create noise for analytic PET simulations. STIR is an open-source software package that is used for tomographic image reconstruction. Motion was simulated in 9 times-frames $(30,60,80,90,120,150,180,240$, or $360 \mathrm{~s})$ with $\pm 0.9375 \mathrm{~cm}(\sim 1 \mathrm{~cm})$ or $\pm 1.8750 \mathrm{~cm}(\sim 2 \mathrm{~cm})$ motion in 6 directions ( $+x$ right, $-x$ left, $+y$ posterior, $-y$ anterior, $+z$ superior,-z inferior). An example of inter-frame motion between two frames in a noisy simulation can be seen in Figure 5.5 and noise-free simulation is shown Figure 5.4.

Time Frame: 13

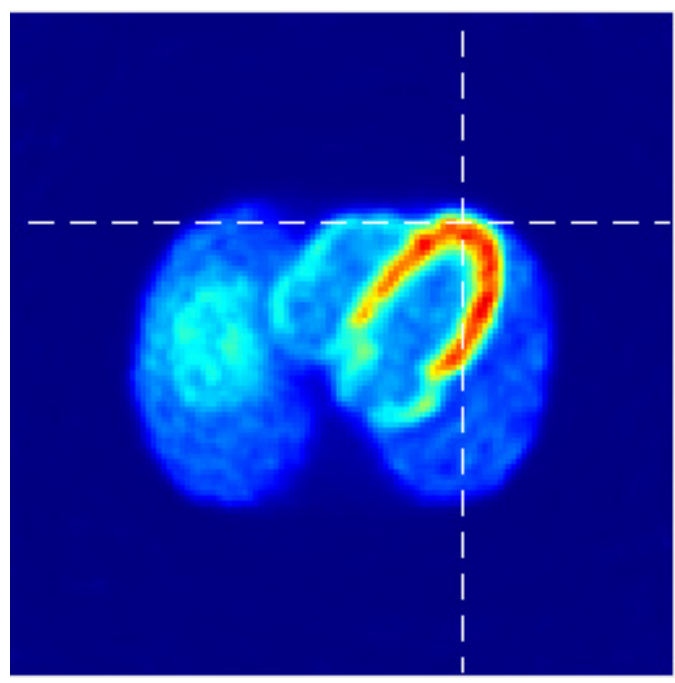

Time Frame: 14

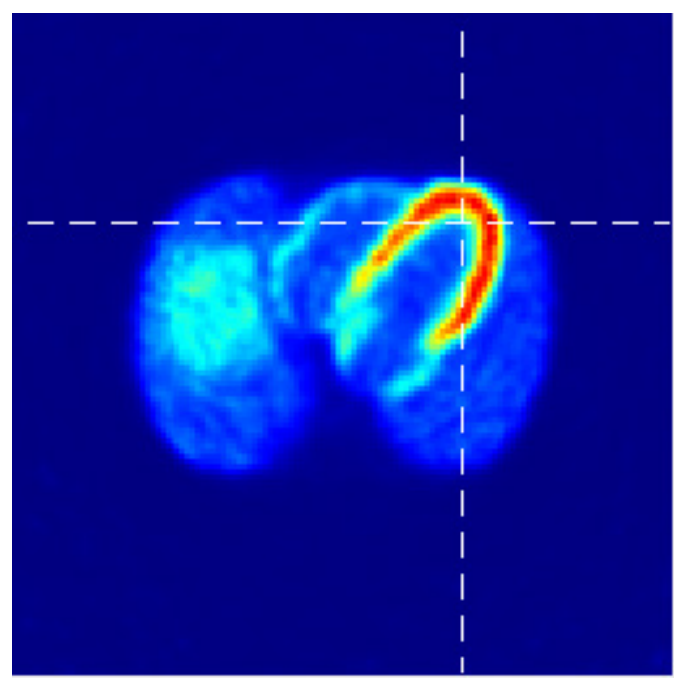

$\operatorname{Max}$

$\operatorname{Max}$

Figure 5.5 - The transverse image shows an NCAT noisy simulation. The image on the left is with no motion and the image on the right is shifted by $2 \mathrm{~cm}$ in the +y direction (at $240 \mathrm{~s}$ ). The myocardium is shown in red. The crosshairs were not shifted between frames. 


\subsubsection{Patient Data}

The same patient images as in section 3.3.1 were used with visual interpretation used as reference truth for the presence of motion. Unlike the simulation data, motion could occur mid-frame, be in any direction and magnitude, and have more than one motion event in a dynamic image sequence. Furthermore, patient images are degraded by photon scatter, random events, noise and attenuation mis-registration (especially in the presence of motion).

The 130 patient images (rest and stress) were cropped to a $45 \times 45 \times 35 \mathrm{VOI}$ containing the entire heart and were visually (re-)evaluated using an in-house software program (FlowQuant $\subset$, Ottawa, ON) $[14,19]$ for the presence of any (mild and significant) interframe heart motion. Any visible heart motion recorded including mild ( $<2$ pixel) and significant ( $\geq 2$ pixel) movement of the heart in the dynamic PET image between neighbouring frames. The time frame of heart motion was recorded on a frame-byframe basis. Inter-frame motion occurs between two successive frames therefore it cannot be detected in time frame 1 . The time frames and their corresponding start time and length in seconds is show in Table 3.1. 


\subsubsection{Image analysis}

In each image set the heart was isolated within a $45 \times 45 \times 35$ VOI for all noise-free, noisy and patient images. All the image sets were decomposed in FADSTool (University of Ottawa Heart Institute, Ottawa, ON) using PCA where the number of factors for decomposition were automatically determine so the components accounted for $\geq 95 \%$ variance in the images sequence. The residue images were then visually inspected for any prominent patterns resembling the myocardium using the same interactive viewing software as used to assess motion in the patient data.

\subsubsection{Automatic Motion Detection Using Quantification of Residue Signal}

The root mean square (RMS) of the residue activity in each time frame was used as a metric to automatically detect the presence of motion. RMS-time curves for noise-free simulations were analyzed for severe and quick changes in amplitude when motion was simulated. Figure 5.6 (top) demonstrates a plot of residue RMS values for each time frame for the case of $+x 2 \mathrm{~cm}$ motion introduced between time frame 3-4. The maximum RMS was about $225 \mathrm{kBq} / \mathrm{cc}$ while the intensity in the surrounding frames is less than $60 \mathrm{kBq} / \mathrm{cc}$ presenting as a sharp peak of residual intensity. A similar observation is made in Figure 5.6 (middle) where inter-frame motion is simulated between time frame 10-11. A less prominent maximum RMS peak is visible in the middle time frames (9 and 10) compared to earlier time frames (4 and 7). Figure 5.6 (bottom) shows motion that was introduced between time frame 14-15. Since image decomposition is done on a single frame and not between frames, motion would be 
detected in the frame following the inter-frame motion event. For example, if motion occurred between time frame 3 and 4 the motion event should be detected in time frame 4 . Therefore in early and middle time frames (1-11) the maximum RMS peak was present in the previous frame to the motion event so it was concluded that an offset of one time frame was required for these time frames. A script was written which calculated the maximum RMS value out of the 15 time frames per residue image. All images were processed through this residue anaylsis script and the prevalence and time of motion were recorded and compared to the baseline (simulated or visual assesment). 


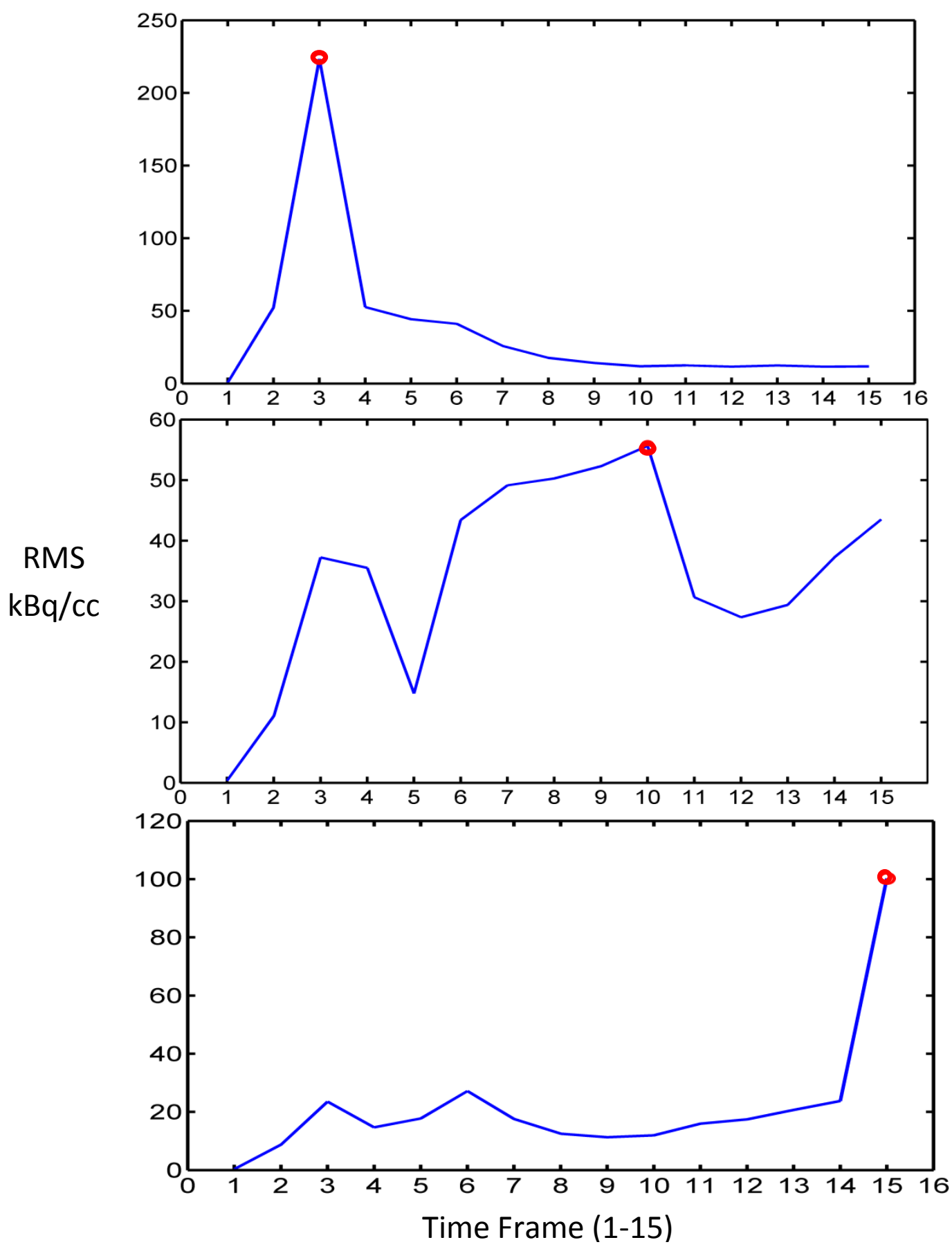

Figure 5.6 - RMS of activity in each of the $\mathbf{1 5}$ time frames of noise-free simulation images. Top: Motion was introduced between time frame 3-4 in the $+x$ direction with a magnitude of $2 \mathrm{~cm}$. Middle: Motion was introduced between time frame 1011 in the $+x$ direction with a magnitude of $2 \mathrm{~cm}$. Bottom: Motion was introduced between time frame 14-15 in the $+x$ direction with a magnitude of $2 \mathrm{~cm}$. The red circle represents the maximum RMS value 


\subsection{Results}

\subsubsection{Noise-free Simulation}

Figure 5.7 demonstrates dynamic sequences of transaxial slices of residual images for noise-free simulations with motion $(+2 \mathrm{~cm} \times$ direction) at different time intervals. The pattern of seeing a high intensity region (red), medium intensity region (yellow) and a low intensity region (blue) together is consistently seen in the frames before or after motion is introduced. Motion in early time frame (4) and late time frames (14 and 15) had better pre-motion vs. post-motion frame contrast compared to motion in the middle time frames (e.g. motion at 7 or 9). As expected, structures resembling that of blood pool (early time frames) and myocardial uptake (later time frames) were more visually evident when dealing with $2 \mathrm{~cm}$ motion in comparison to $1 \mathrm{~cm}$ motion (data not shown). 


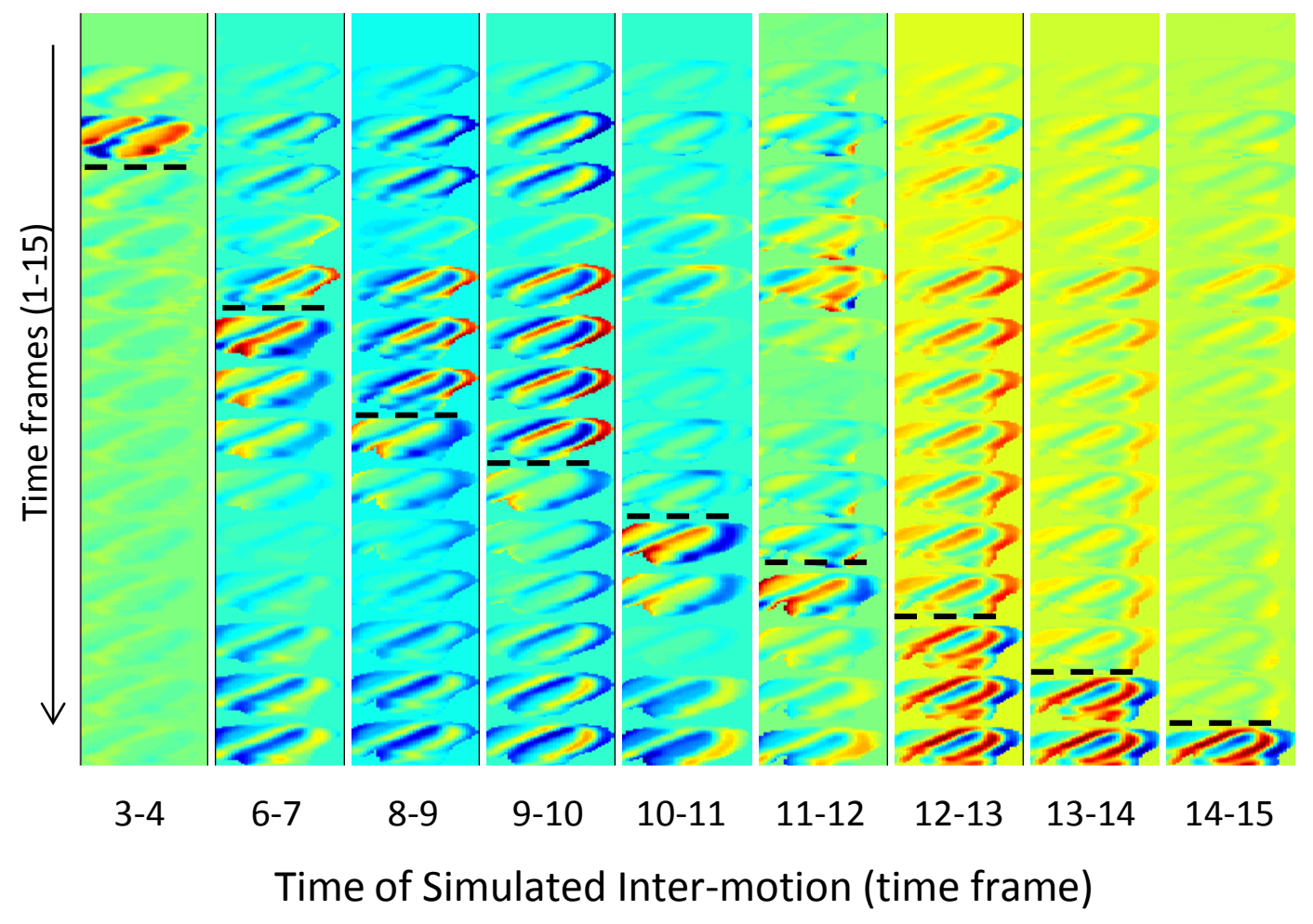

Min

Pixel Intensity

$\operatorname{Max}$

Figure 5.7 - Residue of noise-free NCAT phantoms with simulated motion $(+2 \mathrm{~cm} \mathrm{X}$ direction) at 30s, 60s, 80s, 90s, 120s, 150s, 180s, 240s and 360s represented by the dashed line each in residue. Blood pool (early time frames) and the myocardium (later time frames) is present before or after motion is introduced. Each image is individually scaled between minimum and maximum intensity pixels.

Table 5.1 summarizes the results from image sequence residue analysis with $1 \mathrm{~cm}$ simulated noise-free inter-frame motion events. 42 inter-frame motion events were accurately detected while all 54 motion events were detected within \pm 2 time frames. Likewise, Table 5.2 summarizes the results using $2 \mathrm{~cm}$ motion. 52 inter-frame motion 
events were accurately detected while all 54 motion events were detected within \pm 2

time frames.

Table 5.1 - Detected inter-frame motion events in $1 \mathrm{~cm}$ noise-free simulated motion events. The bolded values account for perfect agreement between residue analysis and visual assessment. The highlighted area accounts for \pm 2 time frames. $N=54$. Note: Frame $\mathbf{2}$ and $\mathbf{3}$ had no motion so are not shown and inter-frame motion cannot occur in time frame 1.

\begin{tabular}{|c|c|c|c|c|c|c|c|c|c|c|c|c|c|}
\hline & \multicolumn{12}{|c|}{ Detected Motion (time frame) } \\
\hline & & 4 & 5 & 6 & 7 & 8 & 9 & 10 & 11 & 12 & 13 & 14 & 15 \\
\hline & 4 & 6 & 0 & 0 & 0 & 0 & 0 & 0 & 0 & 0 & 0 & 0 & 0 \\
\hline & 7 & 0 & 0 & 0 & 6 & 0 & 0 & 0 & 0 & 0 & 0 & 0 & 0 \\
\hline Simulated & 9 & 0 & 0 & 0 & 6 & 0 & 0 & 0 & 0 & 0 & 0 & 0 & 0 \\
\hline $1 \mathrm{~cm}$ Motion & 10 & 0 & 0 & 0 & 0 & 0 & 0 & 0 & 6 & 0 & 0 & 0 & 0 \\
\hline (time frame) & 11 & 0 & 0 & 0 & 0 & 0 & 0 & 0 & 6 & 0 & 0 & 0 & 0 \\
\hline & 12 & 0 & 0 & 0 & 0 & 0 & 0 & 0 & 0 & 6 & 0 & 0 & 0 \\
\hline & 13 & 0 & 0 & 0 & 0 & 0 & 0 & 0 & 0 & 0 & 6 & 0 & 0 \\
\hline & 14 & 0 & 0 & 0 & 0 & 0 & 0 & 0 & 0 & 0 & 0 & 6 & 0 \\
\hline & 15 & 0 & 0 & 0 & 0 & 0 & 0 & 0 & 0 & 0 & 0 & 0 & 6 \\
\hline
\end{tabular}

Table 5.2 - Detected inter-frame motion events in $2 \mathrm{~cm}$ noise-free simulated motion events. The bolded values account for perfect agreement between residue analysis and visual assessment. The highlighted area accounts for \pm 2 time frames. $N=54$

\begin{tabular}{|c|c|c|c|c|c|c|c|c|c|c|c|c|c|}
\hline & \multicolumn{12}{|c|}{ Detected Motion (Time Frame) } \\
\hline & & 4 & 5 & 6 & 7 & 8 & 9 & 10 & 11 & 12 & 13 & 14 & 15 \\
\hline & 4 & 6 & 0 & 0 & 0 & 0 & 0 & 0 & 0 & 0 & 0 & 0 & 0 \\
\hline & 7 & 0 & 0 & 0 & 6 & 0 & 0 & 0 & 0 & 0 & 0 & 0 & 0 \\
\hline Simulated & 9 & 0 & 0 & 0 & 0 & 0 & 6 & 0 & 0 & 0 & 0 & 0 & 0 \\
\hline $2 \mathrm{~cm}$ Motion & 10 & 0 & 0 & 0 & 0 & 0 & 0 & 6 & 0 & 0 & 0 & 0 & 0 \\
\hline (Time Frame) & 11 & 0 & 0 & 0 & 0 & 0 & 0 & 0 & 6 & 0 & 0 & 0 & 0 \\
\hline & 12 & 0 & 0 & 0 & 0 & 0 & 0 & 0 & 0 & 6 & 0 & 0 & 0 \\
\hline & 13 & 0 & 0 & 0 & 0 & 0 & 0 & 0 & 0 & 0 & 4 & 2 & 0 \\
\hline & 14 & 0 & 0 & 0 & 0 & 0 & 0 & 0 & 0 & 0 & 0 & 6 & 0 \\
\hline & 15 & 0 & 0 & 0 & 0 & 0 & 0 & 0 & 0 & 0 & 0 & 0 & 6 \\
\hline
\end{tabular}




\subsubsection{Noisy Simulation}

In noisy simulations a structure resembling the myocardium was visible in the residue image in the frames where motion was present, similar in nature to the noise-free results. However, residue images (Figure 5.8) had more noise and less contrast compared to noise-free simulations (Figure 5.7). As with the noise-free simulations PCA decomposition was most effective when motion was present in early (4) and late time frames $(14,15)$ with good myocardium-to-background contrast. Structures resembling blood pool or the myocardium were visible in all time frames before or after the introduction of motion (denoted by dashed line in Figure 5.8). In middle time frames (7, $9,10,11,12,13)$ the myocardium-like pattern was visible in most of the time frames before and after motion was introduced making localization of the time of motion nontrivial. 


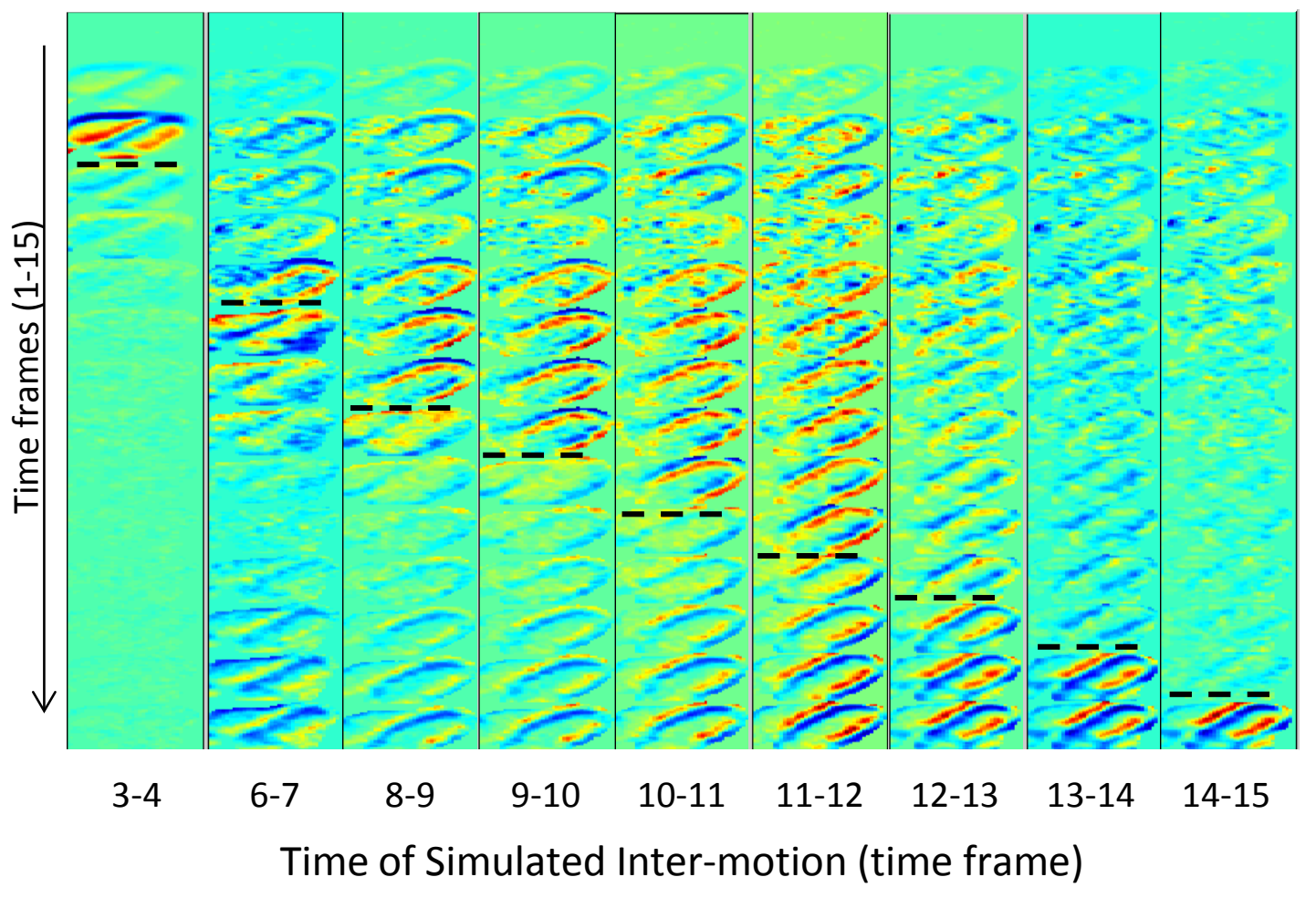

Min _... Pixel Intensity _... .... Max

Figure 5.8 - Example Residue of noisy NCAT phantoms with simulated motion at 30, $60,80,90,120,150,180,240$ and 360 s represented by the dashed line in each residue. Blood pool (early time frames) and the myocardium (later time frames) like patterns are present before or after the motion event.

Table 5.3 and Table 5.4 summarize the automatic motion detection results using 1 and 2 $\mathrm{cm}$ motion respectively. Of the 54 noisy simulations with $1 \mathrm{~cm}$ motion, 28 inter-frame motion events were accurately detected while 50 motion events were detected within \pm 2 time frames. With $2 \mathrm{~cm}$ motion, 38 inter-frame motion events were accurately detected while 49 motion events were detected within \pm 2 time frames. 
Table 5.3 - Detected inter-frame motion events in $1 \mathrm{~cm}$ noisy simulated motion events. The bolded values account for perfect agreement between residue analysis and visual assessment. The highlighted area accounts for \pm 2 time frames. $N=54$

mulated $1 \mathrm{~cm}$ Motion (time frame)

\section{Detected Motion (time frame)}

\begin{tabular}{cr|rrrrrrrrrrrr} 
& & $\mathbf{4}$ & $\mathbf{5}$ & $\mathbf{6}$ & $\mathbf{7}$ & $\mathbf{8}$ & $\mathbf{9}$ & $\mathbf{1 0}$ & $\mathbf{1 1}$ & $\mathbf{1 2}$ & $\mathbf{1 3}$ & $\mathbf{1 4}$ & $\mathbf{1 5}$ \\
\cline { 2 - 11 } Simulated & $\mathbf{4}$ & $\mathbf{6}$ & 0 & 0 & 0 & 0 & 0 & 0 & 0 & 0 & 0 & 0 & 0 \\
$\mathbf{1}$ cm Motion & $\mathbf{7}$ & 0 & 0 & 0 & $\mathbf{6}$ & 0 & 0 & 0 & 0 & 0 & 0 & 0 & 0 \\
(time frame) & $\mathbf{9}$ & 0 & 0 & 0 & 6 & 0 & 0 & 0 & 0 & 0 & 0 & 0 & 0 \\
& $\mathbf{1 1}$ & 0 & 0 & 0 & 0 & 0 & 0 & 0 & 6 & 0 & 0 & 0 & 0 \\
& $\mathbf{1 1}$ & 0 & 0 & 0 & 0 & 0 & 6 & 0 & 0 & 0 & 0 & 0 & 0 \\
& $\mathbf{1 2}$ & 0 & 0 & 0 & 3 & 0 & 0 & 0 & 0 & 0 & 0 & 3 & 0 \\
& 13 & 0 & 0 & 0 & 1 & 0 & 0 & 0 & 0 & 0 & $\mathbf{4}$ & 1 & 0 \\
& 14 & 0 & 0 & 0 & 0 & 0 & 0 & 0 & 0 & 0 & 0 & $\mathbf{6}$ & 0 \\
& 15 & 0 & 0 & 0 & 0 & 0 & 0 & 0 & 0 & 0 & 0 & 0 & $\mathbf{6}$
\end{tabular}

Table 5.4 - Detected inter-frame motion events in $2 \mathrm{~cm}$ noisy simulated motion events. The bolded values account for perfect agreement between residue analysis and visual assessment. The highlighted area accounts for \pm 2 time frames. $\mathrm{N}=54$

\begin{tabular}{cr|rrrrrrrrrrrr} 
& \multicolumn{10}{c|}{ Detected Motion (time frame) } \\
& & $\mathbf{4}$ & $\mathbf{5}$ & $\mathbf{6}$ & $\mathbf{7}$ & $\mathbf{8}$ & $\mathbf{9}$ & $\mathbf{1 0}$ & $\mathbf{1 1}$ & $\mathbf{1 2}$ & $\mathbf{1 3}$ & $\mathbf{1 4}$ & $\mathbf{1 5}$ \\
\hline \multirow{4}{*}{ Simulated } & $\mathbf{4}$ & $\mathbf{6}$ & 0 & 0 & 0 & 0 & 0 & 0 & 0 & 0 & 0 & 0 & 0 \\
$\mathbf{2}$ cm Motion & $\mathbf{7}$ & 0 & 0 & 0 & $\mathbf{6}$ & 0 & 0 & 0 & 0 & 0 & 0 & 0 & 0 \\
(time frame) & $\mathbf{9}$ & 0 & 0 & 0 & 4 & 0 & $\mathbf{2}$ & 0 & 0 & 0 & 0 & 0 & 0 \\
& $\mathbf{1 0}$ & 0 & 0 & 0 & 2 & 0 & 0 & $\mathbf{4}$ & 0 & 0 & 0 & 0 & 0 \\
& $\mathbf{1 1}$ & 0 & 0 & 0 & 3 & 0 & 0 & 1 & $\mathbf{2}$ & 0 & 0 & 0 & 0 \\
& $\mathbf{1 2}$ & 0 & 0 & 0 & 0 & 0 & 0 & 0 & 0 & 0 & 0 & 6 & 0 \\
& $\mathbf{1 3}$ & 0 & 0 & 0 & 0 & 0 & 0 & 0 & 0 & 0 & $\mathbf{6}$ & 0 & 0 \\
& $\mathbf{1 4}$ & 0 & 0 & 0 & 0 & 0 & 0 & 0 & 0 & 0 & 0 & $\mathbf{6}$ & 0 \\
& $\mathbf{1 5}$ & 0 & 0 & 0 & 0 & 0 & 0 & 0 & 0 & 0 & 0 & 0 & $\mathbf{6}$
\end{tabular}

\subsubsection{Patient data}

This study consisted of 130 images (rest and stress) from 65 patients. All patients had at least one motion event at varying magnitudes and direction. In total there were 328 inter-frame heart motion events which were visually detected out of 1820 possible frame pairs. Table 5.5 compares the automatically report time of motion event with 
visually interpreted data. In cases with more than one visually detected motion event the closest visually assessed motion event was used. Of 130 images 60 events were accurately localizes to the correct time frame while 115 motion events were detected within \pm 2 time frames.

Table 5.5 - Automatic temporal localization of inter-frame motion events vs visual assessment. The bolded values account for perfect agreement between residue analysis and visual assessment. The highlighted area accounts for \pm 2 time frames. $\mathbf{N}=130$. Note: inter-frame motion cannot occur in time frame 1

\begin{tabular}{|c|c|c|c|c|c|c|c|c|c|c|c|c|c|c|c|}
\hline & \multicolumn{14}{|c|}{ Detected Motion (time frame) } \\
\hline & & 2 & 3 & 4 & 5 & 6 & 7 & 8 & 9 & 10 & 11 & 12 & 13 & 14 & 15 \\
\hline & 2 & 7 & 0 & 0 & 0 & 0 & 0 & 0 & 0 & 0 & 0 & 0 & 0 & 0 & 0 \\
\hline & 3 & 1 & 0 & 0 & 0 & 0 & 0 & 0 & 0 & 0 & 0 & 0 & 1 & 0 & 0 \\
\hline & 4 & 2 & 1 & 1 & 0 & 0 & 0 & 0 & 0 & 0 & 0 & 1 & 0 & 0 & 0 \\
\hline & 5 & 0 & 0 & 0 & 0 & 0 & 0 & 0 & 0 & 0 & 0 & 0 & 0 & 0 & 0 \\
\hline & 6 & 0 & 0 & 0 & 0 & 0 & 0 & 01 & 0 & 0 & 0 & 0 & 0 & 0 & 1 \\
\hline temporal & 7 & 0 & 0 & 0 & 0 & 0 & 0 & 0 & 0 & 0 & 0 & 0 & 0 & 0 & 0 \\
\hline localization of & 8 & 0 & 0 & 0 & 0 & 0 & 0 & 1 & 0 & 0 & 0 & 0 & 0 & 0 & 0 \\
\hline motion event & 9 & 1 & 0 & 0 & 1 & 0 & 0 & 0 & 0 & 1 & 1 & 0 & 0 & 0 & 0 \\
\hline (time frame) & 10 & 0 & 0 & 0 & 0 & 0 & 0 & 0 & 0 & 0 & 5 & 1 & 2 & 0 & 0 \\
\hline & 11 & 2 & 0 & 0 & 0 & 0 & 0 & 0 & 0 & 0 & 12 & 8 & 1 & 0 & 0 \\
\hline & 12 & 0 & 0 & 0 & 0 & 0 & 0 & 0 & 0 & 0 & 2 & 11 & 13 & 0 & 0 \\
\hline & 13 & 0 & 0 & 0 & 0 & 0 & 0 & 0 & 0 & 0 & 1 & 4 & 20 & 2 & 0 \\
\hline & 14 & 2 & 0 & 0 & 0 & 0 & 0 & 0 & 0 & 0 & 3 & 1 & 6 & 1 & 2 \\
\hline & 15 & 0 & 0 & 0 & 0 & 0 & 0 & 0 & 0 & 0 & 0 & 1 & 1 & 2 & 7 \\
\hline
\end{tabular}

\subsection{Discussion}

To our knowledge, this work explores a completely novel motion detection strategy based on analysis of residual signals during dynamic image decomposition. As such, the results describe herein are a preliminary evaluation of the method based on simulations and patient data. While visual interpretation of the residual image was used to 
scrutinize and understand motion related patterns, an automated method to temporally localize the motion event was also evaluated.

In the noise-free simulations all 108/108 Inter-frame motion events were accurately detected to within \pm 2 time frame. Once noise was introduced into the simulations accuracy decreased, but nevertheless $99 / 108$ motion events were detected within \pm 2 time frame. Of these, motion was detected within \pm 2 time frame for $49 / 54$ cases with 2 $\mathrm{cm}$ motion compared to $50 / 54$ for $1 \mathrm{~cm}$ motion. In a representative clinical patient dataset consisting of 130 images with some level of motion, only 115/328 (35\%) motion events were detected within \pm 2 time frame. This is due, in part, to the automatic detection criterion (maximum RMS) only being able to detect a single heart motion event in a given image sequence. Many of the 130 patient images, however, had multiple times frames containing heart motion.

Thus we concluded that even though the performance of PCA residue image based motion detection may be adversely affected by image noise, preliminary performance measurements are encouraging and further investigation of the technique may be warranted. Nevertheless, some concerns remain.

\subsubsection{Number of events}

The proposed algorithm always detects a single motion event per image. Therefore, it is not clear how the algorithm performs with more than one motion event in a single image or in the absence of motion. In the case of patient images with multiple motion 
events, we elected to use the visually determined motion event closest in frame count to the automatically detected motion event. This strategy clearly biases the results, producing higher accuracy rates.

A strategy to distinguish between absence/presence of motion was not evaluated as part of this work but would be essential for clinical application. Residue analysis would likely be a first part of a multistep process for discriminating motion events.

\subsubsection{Event timing}

In simulation data motion events in early (4) and later time frames (11-15) were detected most robustly while mid-scan events were frequently missed. These findings were confirmed using the patient data. This could be due to the rapidly changing tracer distribution (from blood to tissue) in the middle time frames, making visual assess of heart motion more challenging. Tracer uptake and retention by the myocardium as seen in the later time frames produces a more consistent structure to track.

\subsubsection{Number of Components}

In this work we selected the number of decomposition factors so as to account for $\geq 95 \%$ of the image variance. Figure 5.9 demonstrates the residue of PCA decompositions, using 2, 3, 4 and 5 components. The dynamic image had $2 \mathrm{~cm}$ motion simulated at $240 \mathrm{~s}$ (between time frame 13 and 14 ) in the $+X$ direction. A myocardium shaped pattern is clearly present in time frames 14 and 15 when decomposing with either 2 or 5 factors. While a myocardium shaped residue was present using 3 and 4 factors, it is spread over 
many time frames (both pre- and post-motion event), making it difficult to resolve the timing of the motion event. From this example, it is evident that the number of factors could have a large impact on the motion detection algorithm. From our experience, each image may have a unique number of factors in which the myocardium -tobackground contrast in the residue image is optimized.

Thus further considerations should be given to the number of factors. It may also be possible that the number of factors required to account for a target image variance are indicative of the presence of motion.

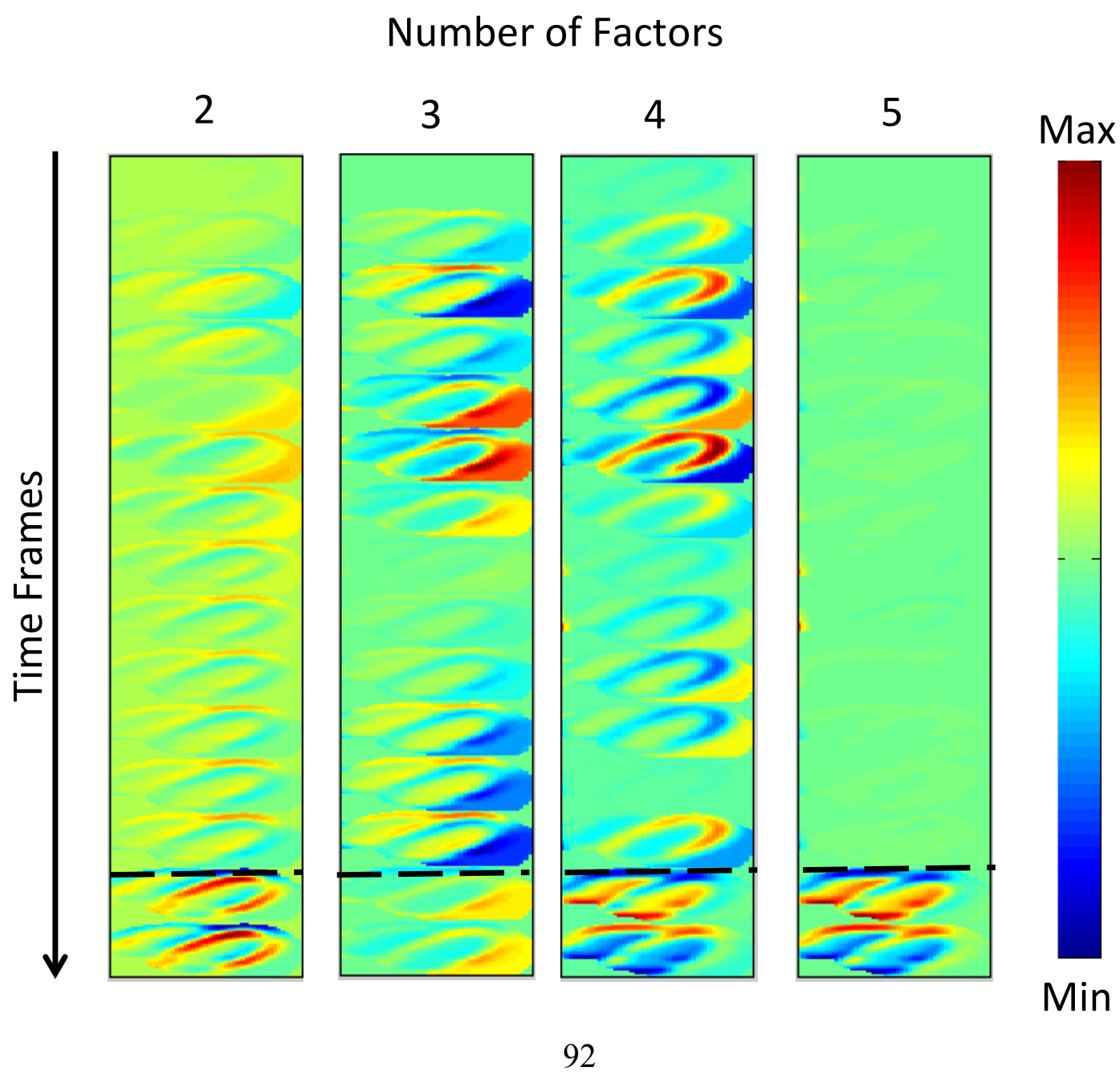

Figure 5.9 - Residue of PCA decomposition using 2, 3, 4 and 5 factors. The noise-free simulation consisted of $2 \mathrm{~cm}$ motion introduced at $240 \mathrm{~s}$ (dashed lined)(between time frame 13-14) in the $+x$ direction 


\subsubsection{Metrics of Motion}

In this work, we relied on RMS of the residue frames to signal the presence of motion. Other possible metrics that may be useful to signify the presence of motion were evaluated in this work including minimum and maximum pixel intensities in the residue images. These metrics were out performed by the RMS metric and their results have been left out of this report for brevity. Nevertheless other metrics may be effective for this purpose. Future work should consider analysis of the Eigenvalues and number of components required account for a target image variance. Another potentially interesting metrics include the entropy or structural information in the residue images as opposed to random noise distribution.

\subsubsection{Limitations and Future Work}

This work is preliminary in nature and therefore may be seen to have numerous limitations. The proposed motion detection algorithm assumes the presence of a single motion event in a dynamic image sequence and as such does not account for images without motion or those with multiple motion events. While this study focused on the merit of using image decomposition to detect heart motion it is evident that for practical application these limitations must be addressed. It may be possible to threshold the RMS value to separate cases with and without heart motion. 
The magnitude of heart motion was not evaluated but further evaluation could provide us with a motion threshold in which this detection method can accurately detect motion.

Furthermore, intra-frame heart motion was not evaluated in this study so as to simplify the analysis to discrete time points in the dynamic image sequence. Future work should include intra-frame motion to improve the accuracy of the simulation.

\subsection{Conclusion}

Preliminary data suggests that heart organ motion detection in dynamic PET using image decomposition may be feasible, especially in the early and late time frames. Further work is required to translate this method into a clinically applicable tool for motion detection and correction. 


\section{Summary}

\subsection{Contributions}

CAD is one of the leading causes of mortality and morbidity in western society and is increasing in prevalence in developing societies [3]. With proper diagnosis and intervention however, quality of life and prognosis can be increased significantly. Currently, MPI is regularly used in clinical practice to detect CAD but may provide falsenegative results in severe cases where narrowing of multiple coronary arteries is present and in diffused disease of the microvasculature $[4,75]$. In both cases the patient may have a uniform decrease in perfusion, in which case relative uptake images may have a uniform distribution consistent with healthy distribution. This limitation can be overcome using absolute MBF quantification [19] which quantifies the amount of blood flow to the myocardium in units of $\mathrm{mL} / \mathrm{min} / \mathrm{g}(\mathrm{mL} / \mathrm{min}$ of blood per gram of tissue). MBF quantification can be performed at rest and stress cardiac states and myocardial flow reserve (MFR) can be calculates as stress/rest ratio. MFR provides incremental prognostic value to physicians when dealing with patients with suspected cases of CAD $[14,20]$.

The gold standard non-invasive modality for MBF quantification is positron emission tomography (PET) [4]. Dynamic cardiac PET images can image the distribution of tracer from the blood stream into the perfused tissue. Sampling of regions of interest (ROIs) over the dynamic sequence produces time-activity curves (TACS) of the changes in 
tracer concentration over time in the anatomical region being sampled. MBF is quantified by analysing the relationship between arterial blood (which perfuses the myocardium) and myocardium TACs. Dynamic imaging implicitly assumes that an ROI corresponds to a specific anatomical location in the patient at all time frames. This assumption is violated by the presence of motion and may manifest itself as distorted TACs which lead to errors in MBF quantification measurements. Therefore the detection of gross patient motion and heart motion has clinical value for image interpretation and quality assurance.

Rigid motion can be present in cardiac imaging for example of the patient shifts during the exams, however heart motion within the thorax is non-rigid as the soft tissues move in relation to one another [35] and tracking of surface markers may not always correlate with heart positioning. Tracking of heart motion in a dynamic cardiac image sequence can also be complicated by changing tracer distributions from blood to perfused tissues. Therefore, heart motion in cardiac PET is a complicated and unresolved problem that can be tackled by several approaches.

One strategy is to correct PET data prior to reconstruction or during reconstruction, but this requires that additional equipment be installed and operated in a fast-paced imaging clinic $[1,2,51,53,54]$. Pre-reconstruction methods generally require that motion data be recorded into the PET camera list-mode data for alignment $[1,2,51,53$, 54]. PET camera vendors, structure and process list-mode data in proprietary ways, making pre-reconstruction strategies non-universal across vendors, and often between 
instrumentation of the same vendor. Post-reconstruction approaches are advantageous in their vendor neutrality and can be simple to process; however not all motion related artifacts (e.g. attenuation mis-registration) may be correctable. Nevertheless, postreconstruction detection of motion may be a useful quality control tool that can be seamlessly incorporated into the MBF quantification workflow. Little work has been done on post-reconstruction non-rigid motion tracking. Gated-PET image acquisition is a well described method but these images are more susceptible to noise due to their decreased photon count $[38,76]$. Fiducial markers have been used to track rigid motion for various imaging applications and other non-rigid motion use pre-reconstruction methods [49-51], however the utility of patient surface mounted fiducial markers for tracking deep organ motion is unclear.

Chapter 3 of this thesis explores whether a positron-emitting fiducial marker motion is a useful surrogate marker of heart motion in dynamic ${ }^{82} \mathrm{Rb}$ PET image sets. A small ${ }^{22} \mathrm{Na}$ marker was affixed to patients' skin over the lower right frontal rib. The marker could be clearly distinguished in the reconstructed PET images. Agreement between presences of motions in the entire dynamic study was $88 \%$ for flagging cases with heart motion based on analysis of fiducial marker motion. In the cases with motion, $16.7 \%$ had motion in different directions and $12.5 \%$ of the images had motion at different time points. Therefore it was concluded that fiducial markers can be useful in predicting the presence of motion but may not be ideal for characterising motion. This method of motion detection was non-invasive and only required the use of a low-cost low-activity 
positron-emitting marker which can be detected in post-reconstructed dynamic PET images. Thus it was concluded that fiducial markers could aid in quality control of dynamic cardiac PET studies, especially if fiducial marker motion detection could be automated.

In Chapter 4 we followed up by developing automatic detection of patient motion using fiducial markers. We focused on two manifestations of motion: inter-frame and intraframe. Inter-frame motion was defined as motion between two successive frames which appears as a sudden shift of the maker between frames bookending the motion event. Intra-frame motion is motion within a single frame, which is manifested as a directional blur of the marker within the affected time frame.

Using visual assessment as our truth reference for motion and ROC analysis, we determined that inter-frame motion could be detected with an accuracy of $91 \%$ using a $2.7 \mathrm{~mm}$ motion threshold. Therefore, the motion detection threshold was smaller than one voxel in size and well below the image spatial resolution. Similarly for intra-frame motion we resolved an optimal activity fall-off rate parameter, B, threshold value of $0.0359 \mathrm{~mm}^{-2}$, which gave an $87 \%$ accuracy estimate This threshold value corresponds to a blur breadth (50\% of peak) of $4.4 \mathrm{~mm}$ which is well below the image spatial resolution. We concluded that these accuracies were sufficiently high to benefit the clinic as a quality control tool when incorporated into the MBF quantification software. 
The advantage automatic tracking of fiducial markers as a surrogate for heart motion is that it is vendor independent, is fast and simple to process, is low-cost and could be easily adapted for a wide range of applications. Nevertheless, this approach is limited by non-perfect correlation between heart and marker motions as described in Chapter 3.

Chapter 5 discusses a completely different approach to heart motion detection, in which the temporal patterns in the heart image itself are analyzed for inconsistencies. The image is decomposed using principle component analysis (PCA) and the residue image (the signal not accounted for by the principal components) is analyzed for patterns that indicate motion. Image decomposition assumes that no motion is present in the image and a violation of this assumption results in additional components being generated or alternatively larger residue intensities and non-random patterns.

Based on a series of simulation and patient based analyses, it was concluded that this method shows promise for detecting inter-frame motion in early and late time frames, but its utility for mid-scan motion is less clear. Furthermore, motion related patterns in the residue became less prominent in the presence of image noise. However, from the patient data we concluded that noise levels do not seem to be a major concern for this application.

Image decomposition as a means of heart motion detection is, as far as we know, a novel approach, and this preliminary research demonstrates that it warrants further investigation. Nevertheless, much more work is required to make image decomposition 
based motion detection more robust and to determine if it can also be used to characterize motion.

\subsection{Limitations}

\subsubsection{Visual Assessment of Motion}

A major limitation of this work is the reliance on visual assessment of images for motion as a reference truth. A quantitative external measurement of the motion would have been preferable especially for validating fiducial marker motion tracking.

Visual evaluation of mild motion of the heart is difficult even with a smaller VOI $(45 \times 45 \times 35)$ due to the constantly changing tracer distribution. This leads to uncertainty of the visually assessed data and a high rate of visually detected cases of suspected heart motion. While mild motion may not be clinically significant it was included in the analysis so as to better understand the robustness of image decomposition based motion detection in a clinical context. Intra-frame motion of the heart is difficult to assess due to the nature of the images and the constantly changing tracer distribution throughout the dynamic cardiac PET image and was therefore was not explicitly addressed. Nevertheless it is safe to assume that many of the images which exhibited inter-frame motion also contained intra-frame motion.

\subsubsection{Simulation Data}

The use of simulation data is an attractive alternative to clinical studies as the motion is known in all its detail. Nevertheless, simulations may not completely model the imaging 
process, patient physiology, and/or motion. While this work utilized noise free and noisy simulations, it only evaluated inter-frame motion and not intra-frame motion. This was partially due to the tediousness and long computer processing time required to generate a large cohort of simulations. While we were not able to complete intra-frame motion simulations in time to include in this work, we are in the process of generating intra-frame motion simulations to further validate the merit in the above discussed motion detection methods.

A further possible improvement over this work could be the use of more accurate simulations including:

- Monte-Carlo simulations of the nuclear physics as opposed to the analytical noise model.

- Addition of attenuation mis-registration artifacts to the motion effected time frames.

- Separate blood TAC for the arterial and venous structures as opposed to a single TAC.

- Cardiac disease models in addition to the healthy (uniform) models in this work.

- Inclusion of rotation motion to the shift motions in this work.

- Inclusion of non-rigid motion (i.e. shifting of the heart within the thorax.

- Inclusion of cardiac and respiratory motions.

- Addition of cases without motion as a negative control cohort 


\subsection{Clinical Feasibility}

Fiducial markers are low in cost and can be applied to patients without significant additional workload to clinical staff. Furthermore, since the markers represent a very low additional radiation dose no special consents would be required for clinical use. Thus, the use of positron-emitting fiducial markers can be clinically adopted immediately with the added benefit of quality control ( $Q C$ ) for motion, though appropriate analysis software would be valuable to fully make use of these markers.

Image decomposition requires no additional costs or equipment during image acquisition. PCA is a fast, well documented and simple decomposition method which can quickly process multiple dynamic images in a short period of time, potentially making it a clinically attractive quality assurance tool. However, interpretation of the results is not straightforward and as such will likely not be clinically beneficial in the immediate future.

Currently, clinics use analysis software to quantify MBF. These methods can easily be integrated into existing software. Alternatively complimentary automated software may be able to run in parallel to the MBF quantification software purely for QC purposes.

The methods described in this work do not correct for motion but could flag patient images where motion is present. If motion is detected then it would warn the observing physician to proceed with caution and if no motion is detected then it increases confidence in the MBF measurements. Steps are being taken to incorporate fiducial 
marker based automatic detection of patient motion in our clinical environment as a quality assurance tool.

\subsection{Other Applications}

Fiducial marker motion tracking has been used for tracking respiratory and tumour motion in PET images pre-reconstruction [50-52]. Fiducial markers have been used with multiple imaging modalities (SPECT, MRI, CT, Ultrasound, X-Ray) for motion tracking [48, 49, 73-77]. Most of these works deal with motion tracking of rigid motion using markers or tracking of lesions; thus the algorithms developed in this work can be adapted for these and other applications. The motion tracking algorithm is designed to track a consistent structure in an image volume sequence not necessary just a spherical fiducial marker in a dynamic PET image. The algorithm can also easily be modified to track motion in a sequence of 2D images. While, dynamic imaging is only a small fraction of imaging done in nuclear medicine it is seeing growth in MRI.

\subsubsection{Motion in Static Images}

The use of a fiducial marker and intra-frame motion detection algorithm could be applied to static MPI images which are acquired over extended time intervals (e.g. 2-8 minutes in PET) and are also susceptible to motion blur artifacts. MPI is much more commonly performed than MBF at the moment and therefore the addition of motion QC could benefit a much larger cohort of patient exams. 


\subsubsection{Multiple marker tracking}

In this work we evaluated the use of a single fiducial marker for detecting the presence of motion. Future work could consist of tracking multiple markers to fully characterize motion and therefore drive patient motion correction. Furthermore, it is possible that multiple marker tracking could improve the accuracy (sensitivity and specificity) of motion detection over the use of a single marker.

\subsubsection{Motion Correction}

While this work evaluated the use of motion detection for quality control, it did not address motion correction. As previously stated, tracking a single fiducial marker does not provide sufficient information for robust motion correction; however with the use of multiple markers this may be possible. The use of image decomposition for motion correction was also not addressed in this work. Motion correction after image reconstruction can effectively reduce MBF error, but may not completely eliminate errors due to uncorrected attenuation mis-registration [47]. Nevertheless, the methods described in this work may be suitable for driving motion correction and therefore may be of interest for future work.

\subsection{Conclusion}

This thesis discusses two potential solutions for automatic motion detection in dynamic PET images. It was concluded that fiducial markers have adequate sensitivity and specificity as predictors of presence of heart motion in dynamic image acquisition. Interand intra-frame fiducial marker motion can accurately be detected automatically. This 
method of heart motion detection should be incorporated into routine clinical use. The final study evaluated the merit of using image decomposition to detect heart motion based on the resulting residue patterns. This method showed promise in detecting cases with motion in noise-free, noisy and clinical patient images especially in early and late time frames but more work is required to determine if it can be useful for routine clinical use. 


\section{Reference}

[1] P. M. Bloomfield, T. J. Spinks, J. Reed, L. Schnorr, A. M. Westrip, L. Livieratos, R. Fulton, and T. Jones, "The design and implementation of a motion correction scheme for neurological PET," Phys. Med. Biol., vol. 48, no. 8, p. 959, Apr. 2003.

[2] B. J. Lopresti, A. Russo, W. F. Jones, T. Fisher, D. G. Crouch, D. E. Altenburger, and D. W. Townsend, "Implementation and performance of an optical motion tracking system for high resolution brain PET imaging," IEEE Trans. Nucl. Sci., vol. 46, no. 6, pp. 2059-2067, Dec. 1999.

[3] P. A. McCullough, "Coronary Artery Disease," Clin. J. Am. Soc. Nephrol., vol. 2, no. 3, pp. 611-616, May 2007.

[4] R. Parkash, R. A. deKemp, T. D. Ruddy, A. Kitsikis, R. Hart, L. Beauschene, K. Williams, R. A. Davies, M. Labinaz, and R. S. B. Beanlands, "Potential utility of rubidium 82 PET quantification in patients with 3-vessel coronary artery disease," J. Nucl. Cardiol., vol. 11, no. 4, pp. 440-449, Jul. 2004.

[5] A. Rahmim and H. Zaidi, "PET versus SPECT: strengths, limitations and challenges:," Nucl. Med. Commun., vol. 29, no. 3, pp. 193-207, Mar. 2008.

[6] T. Beyer, G. Antoch, T. Blodgett, L. F. Freudenberg, T. Akhurst, and S. Mueller, "Dual-modality PET/CT imaging: the effect of respiratory motion on combined image quality in clinical oncology," Eur. J. Nucl. Med. Mol. Imaging, vol. 30, no. 4, pp. 588-596, Feb. 2003.

[7] S. Markus, "Myocardial perfusion imaging with PET.," J. Nucl. Med. Off. Publ. Soc. Nucl. Med., vol. 35, no. 4, pp. 693-698, Apr. 1994.

[8] B. A. Mc Ardle, T. F. Dowsley, R. A. deKemp, G. A. Wells, and R. S. Beanlands, "Does Rubidium-82 PET Have Superior Accuracy to SPECT Perfusion Imaging for the Diagnosis of Obstructive Coronary Disease?A Systematic Review and MetaAnalysis," J. Am. Coll. Cardiol., vol. 60, no. 18, pp. 1828-1837, Oct. 2012.

[9] T. M. Bateman, G. V. Heller, A. I. McGhie, J. D. Friedman, J. A. Case, J. R. Bryngelson, G. K. Hertenstein, K. L. Moutray, K. Reid, and S. J. Cullom, "Diagnostic accuracy of rest/stress ECG-gated Rb-82 myocardial perfusion PET: Comparison with ECG-gated Tc-99m sestamibi SPECT," J. Nucl. Cardiol., vol. 13, no. 1, pp. 2433, Jan. 2006.

[10] F. M. Bengel, T. Higuchi, M. S. Javadi, and R. Lautamäki, "Cardiac Positron Emission Tomography," J. Am. Coll. Cardiol., vol. 54, no. 1, pp. 1-15, Jun. 2009.

[11] T. M. Bateman, "Cardiac positron emission tomography and the role of adenosine pharmacologic stress," Am. J. Cardiol., vol. 94, no. 2, Supplement 1, pp. 19-24, Jul. 2004. 
[12] P. Vaduganathan, Z.-X. He, C. Raghavan, J. J. Mahmarian, and M. S. Verani, "Detection of left anterior descending coronary artery stenosis in patients with left bundle branch block: exercise, adenosine or dobutamine imaging?," J. Am. Coll. Cardiol., vol. 28, no. 3, pp. 543-550, Sep. 1996.

[13] J. Maddahi, "Properties of an ideal PET perfusion tracer: New PET tracer cases and data," J. Nucl. Cardiol., vol. 19, no. 1, pp. 30-37, Jan. 2012.

[14] W. F. Walsh, P. V. Harper, L. Resnekov, and H. Fill, "Noninvasive evaluation of regional myocardial perfusion in 112 patients using a mobile scintillation camera and intravenous nitrogen-13 labeled ammonia.," Circulation, vol. 54, no. 2, pp. 266-275, Aug. 1976.

[15] R. Klein, J. M. Renaud, M. C. Ziadi, S. L. Thorn, A. Adler, R. S. Beanlands, and R. A. deKemp, "Intra- and inter-operator repeatability of myocardial blood flow and myocardial flow reserve measurements using rubidium-82 pet and a highly automated analysis program," J. Nucl. Cardiol., vol. 17, no. 4, pp. 600-616, Apr. 2010.

[16] F. J. Klocke, M. G. Baird, B. H. Lorell, T. M. Bateman, J. V. Messer, D. S. Berman, P. T. O'Gara, B. A. Carabello, J. Russell Richard O., M. D. Cerqueira, M. G. St. John Sutton, A. N. DeMaria, J. E. Udelson, J. W. Kennedy, M. S. Verani, K. A. Williams, E. M. Antman, J. Smith Sidney C., J. S. Alpert, G. Gregoratos, J. L. Anderson, L. F. Hiratzka, D. P. Faxon, S. A. Hunt, V. Fuster, A. K. Jacobs, and R. J. Gibbons, "ACC/AHA/ASNC Guidelines for the Clinical Use of Cardiac Radionuclide ImagingExecutive SummaryA Report of the American College of Cardiology/American Heart Association Task Force on Practice Guidelines (ACC/AHA/ASNC Committee to Revise the 1995 Guidelines for the Clinical Use of Cardiac Radionuclide Imaging)45678," J. Am. Coll. Cardiol., vol. 42, no. 7, pp. 1318-1333, Oct. 2003.

[17] T. A. Holly, B. G. Abbott, M. Al-Mallah, D. A. Calnon, M. C. Cohen, F. P. DiFilippo, E. P. Ficaro, M. R. Freeman, R. C. Hendel, D. Jain, S. M. Leonard, K. J. Nichols, D. M. Polk, and P. Soman, "Single photon-emission computed tomography," J. Nucl. Cardiol., vol. 17, no. 5, pp. 941-973, Oct. 2010.

[18] D. Knollmann, I. Knebel, K.-C. Koch, M. Gebhard, T. Krohn, U. Buell, and W. M. Schaefer, "Comparison of SSS and SRS calculated from normal databases provided by QPS and 4D-MSPECT manufacturers and from identical institutional normals," Eur. J. Nucl. Med. Mol. Imaging, vol. 35, no. 2, pp. 311-318, Feb. 2008.

[19] P. J. Slomka, H. Nishina, D. S. Berman, C. Akincioglu, A. Abidov, J. D. Friedman, S. W. Hayes, and G. Germano, "Automated quantification of myocardial perfusion SPECT using simplified normal limits," J. Nucl. Cardiol. Off. Publ. Am. Soc. Nucl. Cardiol., vol. 12, no. 1, pp. 66-77, Feb. 2005.

[20] R. Klein, R. S. B. Beanlands, and R. A. deKemp, "Quantification of myocardial blood flow and flow reserve: Technical aspects - Springer." [Online]. Available: 
http://link.springer.com.proxy.library.carleton.ca/article/10.1007/s12350-0109256-9. [Accessed: 12-Jul-2015].

[21] M. Lortie, R. S. B. Beanlands, K. Yoshinaga, R. Klein, J. N. DaSilva, and R. A. deKemp, "Quantification of myocardial blood flow with 82Rb dynamic PET imaging," Eur. J. Nucl. Med. Mol. Imaging, vol. 34, no. 11, pp. 1765-1774, Oct. 2007.

[22] M. C. Ziadi, R. A. deKemp, K. Williams, A. Guo, J. M. Renaud, B. J. W. Chow, R. Klein, T. D. Ruddy, M. Aung, L. Garrard, and R. S. B. Beanlands, "Does quantification of myocardial flow reserve using rubidium-82 positron emission tomography facilitate detection of multivessel coronary artery disease?," J. Nucl. Cardiol., vol. 19, no. 4, pp. 670-680, Mar. 2012.

[23] N. P. Johnson and K. L. Gould, "Physiological Basis for Angina and ST-Segment ChangePET-Verified Thresholds of Quantitative Stress Myocardial Perfusion and Coronary Flow Reserve," JACC Cardiovasc. Imaging, vol. 4, no. 9, pp. 990-998, Sep. 2011.

[24] N. P. Johnson and K. L. Gould, "Integrating Noninvasive Absolute Flow, Coronary Flow Reserve, and Ischemic Thresholds Into a Comprehensive Map of Physiological Severity," JACC Cardiovasc. Imaging, vol. 5, no. 4, pp. 430-440, Apr. 2012.

[25] K. Kiso, E. Shimosegawa, H. Watabe, Y. Kanai, K. Fujino, and J. Hatazawa, "Normal Values of Myocardial Blood Flow and Myocardial Flow Reserve Evaluated by 3Dimensional Dynamic PET/CT System with 13N-Ammonia," J. Radiol. Radiat. Ther. Cite.

[26] B. A. Herzog, L. Husmann, I. Valenta, O. Gaemperli, P. T. Siegrist, F. M. Tay, N. Burkhard, C. A. Wyss, and P. A. Kaufmann, "Long-Term Prognostic Value of 13NAmmonia Myocardial Perfusion Positron Emission TomographyAdded Value of Coronary Flow Reserve," J. Am. Coll. Cardiol., vol. 54, no. 2, pp. 150-156, Jul. 2009.

[27] P. G. Coxson, R. H. Huesman, and L. Borland, "Consequences of using a simplified kinetic model for dynamic PET data," J. Nucl. Med., pp. 660-7, 1997.

[28] K. Knešaurek, J. Machac, and Z. Zhang, "Repeatability of regional myocardial blood flow calculation in 82Rb PET imaging," BMC Med. Phys., vol. 9, no. 1, p. 2, 2009.

[29] Y.-H. D. Fang and R. F. Muzic, "Spillover and Partial-Volume Correction for ImageDerived Input Functions for Small-Animal 18F-FDG PET Studies," J. Nucl. Med., vol. 49, no. 4, pp. 606-614, Apr. 2008.

[30] C. Katoh, K. Yoshinaga, O. Manabe, and N. Tamaki, "New quantification algorithm of regional myocardial blood flow with 82Rubidium PET," Soc. Nucl. Med. Annu. Meet. Abstr., vol. 49, no. Supplement 1, p. 384P, May 2008.

[31] H. Gd, C. Jm, and R. Rr, "A region of interest strategy for minimizing resolution distortions in quantitative myocardial PET studies.," J. Nucl. Med. Off. Publ. Soc. Nucl. Med., vol. 33, no. 6, pp. 1243-1250, Jun. 1992. 
[32] E. M. Renkin, "Transport of potassium-42 from blood to tissue in isolated mammalian skeletal muscles," Am. J. Physiol. -- Leg. Content, vol. 197, no. 6, pp. 1205-1210, Dec. 1959.

[33] C. Crone, "The Permeability of Capillaries in Various Organs as Determined by Use of the 'Indicator Diffusion' Method," Acta Physiol. Scand., vol. 58, no. 4, pp. 292305, Aug. 1963.

[34] N. A. Mullani, R. A. Goldstein, K. L. Gould, S. K. Marani, D. J. Fisher, H. A. O’Brien, and M. D. Loberg, "Myocardial perfusion with rubidium-82. I. Measurement of extraction fraction and flow with external detectors," J. Nucl. Med. Off. Publ. Soc. Nucl. Med., vol. 24, no. 10, pp. 898-906, Oct. 1983.

[35] F. Qiao, T. Pan, J. W. C. Jr, and O. R. Mawlawi, "A motion-incorporated reconstruction method for gated PET studies," Phys. Med. Biol., vol. 51, no. 15, p. 3769, Aug. 2006.

[36] Y. Picard and C. J. Thompson, "Motion correction of PET images using multiple acquisition frames," IEEE Trans. Med. Imaging, vol. 16, no. 2, pp. 137-144, Apr. 1997.

[37] P. Buhler, U. Just, E. Will, J. Kotzerke, and J. van den Hoff, "An accurate method for correction of head movement in PET," IEEE Trans. Med. Imaging, vol. 23, no. 9, pp. 1176-1185, Sep. 2004.

[38] N. Dikaios, D. Izquierdo-Garcia, M. J. Graves, V. Mani, Z. A. Fayad, and T. D. Fryer, "MRI-based motion correction of thoracic PET: initial comparison of acquisition protocols and correction strategies suitable for simultaneous PET/MRI systems," Eur. Radiol., vol. 22, no. 2, pp. 439-446, Sep. 2011.

[39] E. J. Gravier and Y. Yang, "Motion-compensated reconstruction of tomographic image sequences," IEEE Trans. Nucl. Sci., vol. 52, no. 1, pp. 51-56, Feb. 2005.

[40] S. A. Nehmeh, Y. E. Erdi, T. Pan, E. Yorke, G. S. Mageras, K. E. Rosenzweig, H. Schoder, H. Mostafavi, O. Squire, A. Pevsner, S. M. Larson, and J. L. Humm, "Quantitation of respiratory motion during 4D-PET/CT acquisition," Med. Phys., vol. 31, no. 6, pp. 1333-1338, Jun. 2004.

[41] G. Lucignani, "Respiratory and cardiac motion correction with 4D PET imaging: shooting at moving targets," Eur. J. Nucl. Med. Mol. Imaging, vol. 36, no. 2, pp. 315-319, Nov. 2008.

[42] A. Pourmoghaddas, R. Klein, R. A. deKemp, and R. G. Wells, "Respiratory phase alignment improves blood-flow quantification in Rb82 PET myocardial perfusion imaging," Med. Phys., vol. 40, no. 2, p. 022503, Feb. 2013.

[43] V. Bettinardi, M. Picchio, N. Di Muzio, L. Gianolli, M. C. Gilardi, and C. Messa, "Detection and compensation of organ/lesion motion using 4D-PET/CT respiratory 
gated acquisition techniques," Radiother. Oncol., vol. 96, no. 3, pp. 311-316, Sep. 2010.

[44] S. A. Nehmeh and Y. E. Erdi, "Respiratory Motion in Positron Emission Tomography/Computed Tomography: A Review," Semin. Nucl. Med., vol. 38, no. 3, pp. 167-176, May 2008.

[45] S. A. Nehmeh, Y. E. Erdi, C. C. Ling, K. E. Rosenzweig, H. Schoder, S. M. Larson, H. A. Macapinlac, O. D. Squire, and J. L. Humm, "Effect of Respiratory Gating on Quantifying PET Images of Lung Cancer," J. Nucl. Med., vol. 43, no. 7, pp. 876-881, Jul. 2002.

[46] Y. E. Erdi, S. A. Nehmeh, T. Pan, A. Pevsner, K. E. Rosenzweig, G. Mageras, E. D. Yorke, H. Schoder, W. Hsiao, O. D. Squire, P. Vernon, J. B. Ashman, H. Mostafavi, S. M. Larson, and J. L. Humm, "The CT Motion Quantitation of Lung Lesions and Its Impact on PET-Measured SUVs," J. Nucl. Med., vol. 45, no. 8, pp. 1287-1292, Aug. 2004.

[47] C. Hunter, R. Klein, and R. deKemp, "Correction for patient body motion in Rb-82 PET-CT: An NCAT simulation study," J. Nucl. Med., vol. 55, no. supplement 1, pp. 2054-2054, May 2014.

[48] R. Klein, C. Hunter, R. Beanlands, and R. deKemp, "Prevalence of patient motion in dynamic PET," Soc. Nucl. Med. Annu. Meet. Abstr., vol. 52, no. Supplement 1, p. 2105, May 2011.

[49] M. Tremblay, F. Tam, and S. J. Graham, "Retrospective coregistration of functional magnetic resonance imaging data using external monitoring," Magn. Reson. Med., vol. 53, no. 1, pp. 141-149, Jan. 2005.

[50] C. Dold, M. Zaitsev, O. Speck, E. A. Firle, J. Hennig, and G. Sakas, “Advantages and Limitations of Prospective Head Motion Compensation for MRI Using an Optical Motion Tracking Device," Acad. Radiol., vol. 13, no. 9, pp. 1093-1103, Sep. 2006.

[51] M. Zaitsev, C. Dold, G. Sakas, J. Hennig, and O. Speck, “Magnetic resonance imaging of freely moving objects: prospective real-time motion correction using an external optical motion tracking system," Neurolmage, vol. 31, no. 3, pp. 1038-1050, Jul. 2006.

[52] S. A. Nehmeh, Y. E. Erdi, C. C. Ling, K. E. Rosenzweig, O. D. Squire, L. E. Braban, E. Ford, K. Sidhu, G. S. Mageras, S. M. Larson, and J. L. Humm, "Effect of respiratory gating on reducing lung motion artifacts in PET imaging of lung cancer," Med. Phys., vol. 29, no. 3, pp. 366-371, Mar. 2002.

[53] M. Chamberland, R. Wassenaar, B. Spencer, and T. Xu, "Performance evaluation of real-time motion tracking using positron emission fiducial markers," Med. Phys., vol. 38, no. 2, pp. 810-819, Feb. 2011. 
[54] T. Xu, J. T. Wong, P. M. Shikhaliev, J. L. Ducote, M. S. Al-Ghazi, and S. Molloi, "Realtime tumor tracking using implanted positron emission markers: Concept and simulation study," Med. Phys., vol. 33, no. 7, pp. 2598-2609, Jul. 2006.

[55] R. Klein, A. Adler, R. S. Beanlands, and R. A. deKemp, "Precision-controlled elution of a ${ }^{82} \mathrm{Sr} /{ }^{82} \mathrm{Rb}$ generator for cardiac perfusion imaging with positron emission tomography," Phys. Med. Biol., vol. 52, no. 3, pp. 659-673, Feb. 2007.

[56] H. Mohy-ud-Din, N. A. Karakatsanis, J. S. Goddard, J. Baba, W. Wills, A. K. Tahari, D. F. Wong, and A. Rahmim, "Generalized dynamic PET inter-frame and intra-frame motion correction - Phantom and human validation studies," in 2012 IEEE Nuclear Science Symposium and Medical Imaging Conference (NSS/MIC), 2012, pp. 30673078.

[57] M. Menke, M. S. Atkins, and K. R. Buckley, "Compensation methods for head motion detected during PET imaging," IEEE Trans. Nucl. Sci., vol. 43, no. 1, pp. 310317, Feb. 1996.

[58] A. I. Veress, W. P. Segars, B. M. W. Tsui, J. A. Weiss, and G. T. Gullberg, "Physiologically realistic LV models to produce normal and pathological image and phantom data," in 2004 IEEE Nuclear Science Symposium Conference Record, 2004, vol. 7, pp. 4231-4235.

[59] A. I. Veress, W. P. Segars, J. A. Weiss, B. M. W. Tsui, and G. T. Gullberg, "Normal and Pathological NCAT Image and Phantom Data Based on Physiologically Realistic Left Ventricle Finite-Element Models," IEEE Trans. Med. Imaging, vol. 25, no. 12, pp. 1604-1616, Dec. 2006.

[60] W. P. Segars, D. S. Lalush, and B. M. W. Tsui, "Development of an interactive software application to model patient populations in the 4D NURBS-based cardiac torso phantom," in 2000 IEEE Nuclear Science Symposium Conference Record, 2000, vol. 3, pp. 20/51-20/55 vol.3.

[61] C. R. R. N. Hunter, J. Hill, M. C. Ziadi, R. S. B. Beanlands, and R. A. deKemp, "Biodistribution and radiation dosimetry of $82 \mathrm{Rb}$ at rest and during peak pharmacological stress in patients referred for myocardial perfusion imaging," Eur. J. Nucl. Med. Mol. Imaging, vol. 42, no. 7, pp. 1032-1042, Mar. 2015.

[62] R. Klein, "Editorial: Derivation of respiratory gating signals from ECG signals," J. Nucl. Cardiol., pp. 1-3, Jun. 2015.

[63] C. Schiepers, C. K. Hoh, J. Nuyts, H.-M. Wu, M. E. Phelps, and M. Dahlbom, "Factor analysis in prostate cancer: delineation of organ structures and automatic generation of in- and output functions," IEEE Trans. Nucl. Sci., vol. 49, no. 5, pp. 2338-2343, Oct. 2002. 
[64] G. E. Fakhri, A. Sitek, B. Guérin, M. F. Kijewski, M. F. D. Carli, and S. C. Moore, "Quantitative Dynamic Cardiac 82Rb PET Using Generalized Factor and Compartment Analyses," J. Nucl. Med., vol. 46, no. 8, pp. 1264-1271, Aug. 2005.

[65] W. Hm, H. Ck, C. Y, S. Hr, H. Ra, P. Me, and H. Sc, "Factor analysis for extraction of blood time-activity curves in dynamic FDG-PET studies.," J. Nucl. Med. Off. Publ. Soc. Nucl. Med., vol. 36, no. 9, pp. 1714-1722, Sep. 1995.

[66] J. Y. Ahn, D. S. Lee, J. S. Lee, S.-K. Kim, G. J. Cheon, J. S. Yeo, S.-A. Shin, J.-K. Chung, and M. C. Lee, "Quantification of Regional Myocardial Blood Flow Using Dynamic H2150 PET and Factor Analysis," J. Nucl. Med., vol. 42, no. 5, pp. 782-787, May 2001.

[67] A. Sitek, E. V. R. D. Bella, and G. T. Gullberg, "Factor analysis with a priori knowledge - application in dynamic cardiac SPECT," Phys. Med. Biol., vol. 45, no. 9, p. 2619, Sep. 2000.

[68] S. Wold, K. Esbensen, and P. Geladi, "Principal component analysis," Chemom. Intell. Lab. Syst., vol. 2, no. 1-3, pp. 37-52, Aug. 1987.

[69] F. Hermansen and A. A. Lammertsma, "Linear dimension reduction of sequences of medical images: I. Optimal inner products," Phys. Med. Biol., vol. 40, no. 11, p. 1909, Nov. 1995.

[70] E. J. Candès, X. Li, Y. Ma, and J. Wright, "Robust Principal Component Analysis?," J ACM, vol. 58, no. 3, pp. 11:1-11:37, Jun. 2011.

[71] H. H. Harman, Modern Factor Analysis. University of Chicago Press, 1976.

[72] R. Klein, "Kinetic Model Based Factor Analysis of Cardiac 82 Rb PET Images for Improved Accuracy of Quantitative Myocardial Blood Flow Measurement," University of Ottawa, 2010.

[73] C. Comtat, P. E. Kinahan, M. Defrise, C. Michel, C. Lartizien, and D. W. Townsend, "Simulating whole-body PET scanning with rapid analytical methods," in 1999 IEEE Nuclear Science Symposium, 1999. Conference Record, 1999, vol. 3, pp. 1260-1264 vol.3.

[74] K. Thielemans, C. Tsoumpas, S. Mustafovic, T. Beisel, P. Aguiar, Nikolaos Dikaios, and M. W. Jacobson, "STIR: software for tomographic image reconstruction release 2," Phys. Med. Biol., vol. 57, no. 4, p. 867, 2012.

[75] N. M. K Yoshida, "Coronary flow and flow reserve by PET simplified for clinical applications using rubidium-82 or nitrogen-13-ammonia," J. Nucl. Med. Off. Publ. Soc. Nucl. Med., vol. 37, no. 10, pp. 1701-12, 1996.

[76] M. Dawood, N. Lang, X. Jiang, and K. P. Schafers, "Lung motion correction on respiratory gated 3-D PET/CT images," IEEE Trans. Med. Imaging, vol. 25, no. 4, pp. 476-485, Apr. 2006. 
[77] A. S. Beddar, K. Kainz, T. M. Briere, Y. Tsunashima, T. Pan, K. Prado, R. Mohan, M. Gillin, and S. Krishnan, "Correlation between internal fiducial tumor motion and external marker motion for liver tumors imaged with 4D-CT," Int. J. Radiat. Oncol., vol. 67, no. 2, pp. 630-638, Feb. 2007.

[78] W. Nawara, B. Vastenhouw, Y. Wang, M. Pomper, and F. Beekman, "Brain motion correction for pre-clinical stationary pinhole SPECT-PET using radioactive fiducial markers.," J. Nucl. Med., vol. 56, no. supplement 3, pp. 1812-1812, May 2015.

[79] J. Maurer C. R., J. M. Fitzpatrick, M. Y. Wang, R. L. Galloway, R. J. Maciunas, and G. S. Allen, "Registration of head volume images using implantable fiducial markers," IEEE Trans. Med. Imaging, vol. 16, no. 4, pp. 447-462, Aug. 1997.

[80] S. K. Ng, E. Armour, L. Su, Y. Zhang, I. Iordachita, J. Wong, H. T. Sen, P. Kazanzides, M. L. Bell, and K. Ding, "SU-E-J-42: Evaluation of Fiducial Markers for Ultrasound and X-Ray Images Used for Motion Tracking in Pancreas SBRT," Med. Phys., vol. 42, no. 6, pp. 3273-3273, Jun. 2015.

[81] W. Wunderink, A. Méndez Romero, W. de Kruijf, H. de Boer, P. Levendag, and B. Heijmen, "Reduction of Respiratory Liver Tumor Motion by Abdominal Compression in Stereotactic Body Frame, Analyzed by Tracking Fiducial Markers Implanted in Liver," Int. J. Radiat. Oncol., vol. 71, no. 3, pp. 907-915, Jul. 2008. 